\title{
Cohesive surface model for fracture based on a two-scale formulation: computational implementation aspects
}

\section{S. Toro, P. J. Sánchez, J. M. Podestá, P. J. Blanco, A. E. Huespe \& R. A. Feijóo}

\section{Computational Mechanics}

Solids, Fluids, Structures, Fluid-

Structure Interactions, Biomechanics, Micromechanics, Multiscale Mechanics, Materials, Constitutive Modeling, Nonlinear Mechanics, Aerodynamics

\section{ISSN 0178-7675}

Volume 58

Number 4

Comput Mech (2016) 58:549-585 DOI 10.1007/s00466-016-1306-y

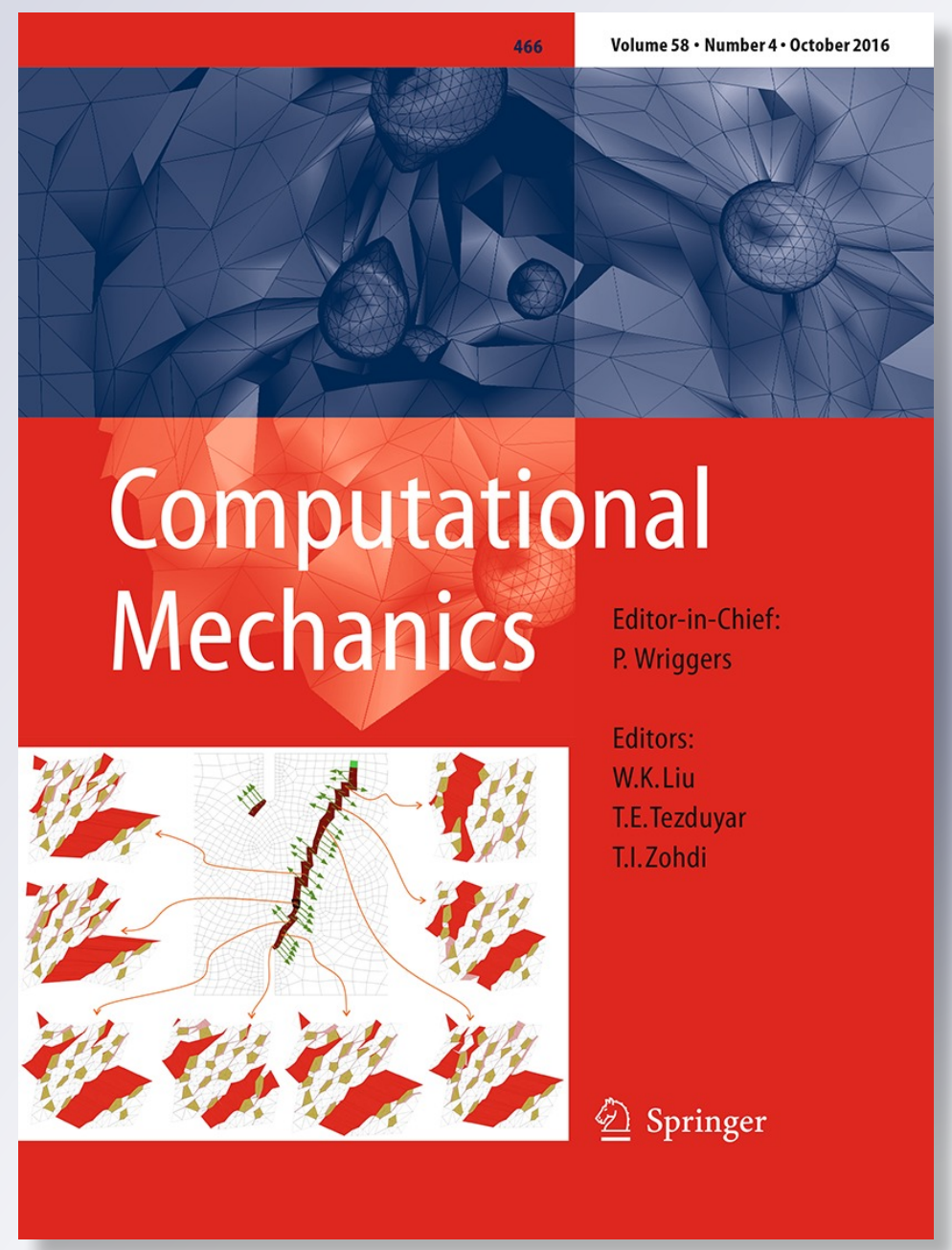

望 Springer 
Your article is protected by copyright and all rights are held exclusively by SpringerVerlag Berlin Heidelberg. This e-offprint is for personal use only and shall not be selfarchived in electronic repositories. If you wish to self-archive your article, please use the accepted manuscript version for posting on your own website. You may further deposit the accepted manuscript version in any repository, provided it is only made publicly available 12 months after official publication or later and provided acknowledgement is given to the original source of publication and a link is inserted to the published article on Springer's website. The link must be accompanied by the following text: "The final publication is available at link.springer.com". 


\title{
Cohesive surface model for fracture based on a two-scale formulation: computational implementation aspects
}

\author{
S. Toro ${ }^{1,2}$ - P. J. Sánchez ${ }^{1,2}$ - J. M. Podestá ${ }^{1}$ P. J. Blanco ${ }^{3,4}$. \\ A. E. Huespe ${ }^{1,5}$ R. A. Feijóo ${ }^{3,4}$
}

Received: 3 December 2015 / Accepted: 25 May 2016 / Published online: 12 July 2016

(C) Springer-Verlag Berlin Heidelberg 2016

\begin{abstract}
The paper describes the computational aspects and numerical implementation of a two-scale cohesive surface methodology developed for analyzing fracture in heterogeneous materials with complex micro-structures. This approach can be categorized as a semi-concurrent model using the representative volume element concept. A variational multi-scale formulation of the methodology has been previously presented by the authors. Subsequently, the formulation has been generalized and improved in two aspects: (i) cohesive surfaces have been introduced at both scales of analysis, they are modeled with a strong discontinuity kinematics (new equations describing the insertion of the macro-scale strains, into the micro-scale and the posterior homogenization procedure have been considered); (ii) the computational procedure and numerical implementation have been adapted for this formulation. The first point has been presented elsewhere, and it is summarized here. Instead, the main objective of this paper is to address a rather detailed presentation of the second point. Finite element techniques
\end{abstract}

A. E. Huespe

ahuespe@intec.unl.edu.ar

1 CIMEC-UNL-CONICET, Güemes 3450, CP 3000 Santa Fe, Argentina

2 GIMNI-UTN-FRSF, Lavaise 610, CP 3000 Santa Fe, Argentina

3 LNCC/MCTI, Laboratório Nacional de Computação Científica, Av. Getúlio Vargas 333, Petrópolis, RJ CEP 25651-075, Brazil

4 INCT-MACC, Instituto Nacional de Ciência e Tecnologia em Medicina Assistida por Computação Científica, Petrópolis, Brazil

5 Centre Internacional de Métodes Numérics en Enginyeria (CIMNE), Campus Nord UPC, Edifici C-1, J. Girona 1-3, 08034 Barcelona, Spain for modeling cohesive surfaces at both scales of analysis ( $\mathrm{FE}^{2}$ approach) are described: (i) finite elements with embedded strong discontinuities are used for the macro-scale simulation, and (ii) continuum-type finite elements with high aspect ratios, mimicking cohesive surfaces, are adopted for simulating the failure mechanisms at the micro-scale. The methodology is validated through numerical simulation of a quasi-brittle concrete fracture problem. The proposed multiscale model is capable of unveiling the mechanisms that lead from the material degradation phenomenon at the mesostructural level to the activation and propagation of cohesive surfaces at the structural scale.

Keywords Multi-scale cohesive models - Computational homogenization - Heterogeneous material failure . Embedded Finite Elements (EFEM)

\section{Introduction}

\subsection{Literature review}

Cohesive surface models (CSM) have proven to be a useful conceptual tool for analyzing material fracture. This approach consists in introducing a surface with two interfaces into the continuum. A relative displacement, or displacement jump, is modeled between both interfaces, while cohesive tractions on the same interfaces react by opposing to their opening. As the interfaces are opened, the cohesive forces decrease, tending to zero, which finally yields a new tractionfree crack into the continuum. So, the generation of a new traction-free crack demands energy from the mechanical system.

CSM are appropriate to describe the nonlinear dissipative effects observed at small length scales, specifically into the 
fracture process zone of a propagating crack. The modeling of these effects requires an adequate characterization of the traction-displacement jump law governing the cohesive surface evolution. Implicitly, this characterization also involves the amount of energy demanded to the mechanical system to generate a new traction-free crack into the solid. So, the selection of the traction-displacement jump law turns out to be the foremost issue for modeling cohesive surfaces.

CSM have been firstly introduced in fracture mechanics by Dugdale [1] and Barenblatt [2]. In computational mechanics, they were introduced by Needleman and co-workers (Needleman [3], Xu and Needleman [4], see also references cited in Needleman [5]). After them, CSM have gained a wide acceptance for several reasons. First, its numerical implementation is simple and efficient. But, mainly, CSM have become popular due to the wide range of problems and applications for which they provide useful solutions. Typical examples of CSM applied to dynamics fracture simulations are shown in Falk et al. [6], Xu and Needleman [7] and Pandolfi et al. [8]. In ductile fracture analysis, CSM have been utilized by Hutchinson and Evans [9], Tvergaard [10], Siegmund and Brocks [11], Huespe et al. [12], Huespe et al. [13]. Furthermore, and from the pioneering works of Hillerborg [14], Bažant [15] and Elices et al. [16], CSM models have been widely applied to the field of quasi-brittle materials for modeling concrete fracture problems.

According to the aim of the present work, some features, assumptions and limitations of classical CSM, mainly those related to the traction-separation law description, are summarized and remarked:

(i) Usually, for modeling quasi-brittle fracture, the constitutive law relating tractions to displacement jumps across the cohesive surface is proposed independently of the continuum constitutive relation characterizing the bulk material (exceptions to this approach have been proposed in $[17,18]$ and references cited therein). Two key parameters govern the response of the cohesive forces: the fracture energy and the peak stress. In practical applications, they are estimated from experiments. However, both parameters depend on phenomena occurring at smaller length scales. Typically, a composite could display noticeable differences of the overall fracture energy by changing its micro-structure topology. Topology changes could happen during the loading process, due to material degradation itself. In this case, the experimental determination of parameters governing the traction-separation law, is not free of controversy. So, an alternative approach for assessing the overall fracture energy consists in modeling the material degradation processes at small length scales, where the fracture energy of every compound can be accurately characterized, simultaneously with the crack propagation problem at the structural level. Then, by using a specific homogenization technique, the overall fracture energy can be estimated in an unified framework of analysis.

(ii) Ductile fracture analysis using CSM has also been widely addressed in the literature. However, in this case, and contrarily to the quasi-brittle fracture approaches, the parameters determining the traction-separation law depend more markedly on the loading path. Typically, separation work in ductile fracture, as well as the peak stress, is enormously influenced by the stress triaxiality (see Tvergaard and Hutchinson [19]). A viable technique to deal with these complex phenomena is the use of multiscale models, adopting cohesive forces on the interfaces and determining the traction-separation law through a micro-structural analysis. Several works have followed this approach, such as Siegmund and Brocks [11]. However, most of them consider a multi-scale technique where the transference of information between scales flows only in one direction, from the smaller length scale to the larger one (hierarchical model). This kind of hierarchical approach may be questionable when the cohesive forces depend strongly on macro-scale variables that change during the structural loading history.

Within the class of multi-scale procedures for modeling ductile fracture, it is suitable to mention the works of Xia and Shih [20] and Vernerey et al. [21]. In both cases, even when the fracture process zone at the macro-scale is not approached through CSM, the effects induced by the micro-structure during the failure process are taken into account. In the work of Xia and Shih, the microstructure is explicitly embedded into the macro-model, while, in the work of Vernerey et al., the micro-structure degradation mechanisms are modeled by increasing the kinematical descriptors at the macro-scale using the micromorphic theory framework. Both approaches result highly motivating techniques for developing the model described in this paper.

Determining the overall constitutive relation of microstructured materials undergoing fracture, via multi-scale procedures, involves meaningful theoretical challenges. In a previous work of the authors, see Sánchez et al. [22], it has been shown that once the macro-scale material response loses the strong ellipticity condition, the conventional homogenization procedure (volume average), evaluated with the use of an RVE, becomes an ill-posed problem, see also Gitman et al. [23] and Nguyen et al. [24]. Contrarily to what happens during the macro-scale stable pre-critical regime, multi-scale problems involving strain localization demand the use of general and original formulations based on non-conventional homogenization paradigms. In last years, this problem has been the motive of intense study. Several authors have contributed with new ideas and theoretical or numerical models. 
To cite only a few of them, we reference the following works: Belytschko et al. [25], Belytschko and Song [26], Geers et al. [27], Bosco et al. [28], Nguyen et al. [29], Verhoosel et al. [30] Oliver et al. [31], Kulkarni et al. [32] and references cited therein.

\subsection{CSM from a novel RVE-based multi-scale framework}

Blanco and co-workers [33] have presented a variationally consistent RVE-based unified theory established on the following axioms: (i) kinematic admissibility, (ii) mathematical duality and (iii) virtual power principle. They have coined the name Method of Multi-scale Virtual Power (MMVP) for identifying this theory. In the MMVP, the physics between the involved length scales are linked by postulating the so-called Principle of Multi-scale Virtual Power (PMVP), which is a generalization of the Hill-Mandel statement of macro-homogeneity [34,35]. By using the MMVP, the homogenization formulas for stress-like quantities, the establishment of the precise boundary conditions imposed to the RVE mechanical model and the micro-scale equilibrium problem, are unambiguously derived as consequences of the variational formulation, in contrast with alternative approaches where they are a-priori assumed.

The general framework proposed in [33] encompasses a wide spectrum of multi-scale modeling scenarios, ranging from conventional constitutive-inspired multi-scale theories (see [36-39]) to more sophisticated and novel approaches, as for example: multi-scale techniques linking high order macro-scale description with standard (first order) microscale kinematics [40], thermo-mechanical models (see [41]), multi-scale formulations accounting for inertia and body forces effects (see [42]), etc.

The MMVP can additionally be used as the underlying theory to derive multi-scale models for material failure based on CSM, where the traction-separation law characterizing the macro-scale cohesive interface is obtained by homogenization of micro-scale degradation mechanisms. For example, in Sánchez et al. [22] and Toro et al. [43], a semi-concurrent two-scale approach for material failure analysis has been proposed. The failure phenomena at the micro-scale are represented by the existence of strain localization bands, modeled with a smeared crack technique.

Also, based on the MMVP concept, Toro et al. [44] have subsequently generalized and improved the methodology proposed in [22] and [43] by introducing cohesive surfaces at both scales of analysis. New equations for transferring information between scales (scale-bridging equations) have been developed and presented. As a further development of the two-scale formulation described in Toro et al. [44], a detailed finite element implementation is described in this article to shed light on the following issues: (i) numerical treatment given to CSM at macro and micro scales, including EFEM finite element technique and crack path tracking algorithm, (ii) detailed procedure to evaluate the homogenization formulae for the macro-scale stresses and cohesive tractions, (iii) implementation of the kinematical restrictions on the RVE model, (iv) implementation of the equilibrium problems at both scales of analysis. The computational tool obtained with this procedure results efficient for simulating a wide range of failure phenomena in heterogeneous materials. This subject represents the main contribution of the present work.

The remaining of the paper is structured as follows: in Sect. 2, we give a brief theoretical description of the methodology formulated in Toro et al. [44]. The main contribution of the present paper is introduced in Sect. 3, where a detailed description of the finite element models used at both scales, macro and micro, is shown. An embedded strong discontinuity finite element technique (EFEM) is proposed to simulate the cohesive surfaces at the macro-scale. Furthermore, a detailed description of the fracture model at micro-scale is also presented in the same section. The numerical assessment of the model is presented in Sect. 4. Sensitivity analysis of the macro-scale response with respect to: (i) mesoscopic cell size and (ii) finite element mesh size of a given specific cell, are shown and discussed. Finally, a structural fracture problem is modeled with an heterogeneous quasi-brittle material. Last Section of the paper is devoted to discuss some conclusions.

\section{Description of the multi-scale mechanical formulation}

According with the objectives pursued in this contribution, a brief overview of the multi-scale mechanical formulation is shown in this Section, which provides the necessary background for describing the computational implementation in the remaining part of the paper. The full model formulation has been described in Toro et al. [44]. Thus, the readers are addressed to that work for obtaining further details of the formulation, as well as to [33] for consulting specific aspects of the MMVP theory.

Notation used in this work Objects intervening in the model are identified as follows (see Fig. 1):

(i) The body domain at the macro-scale is denoted $\Omega$. Points in this domain are identified with $x$. Macroscale fields, in $\Omega$, are: displacements $\boldsymbol{u}(\boldsymbol{x})$, strains $\varepsilon(\boldsymbol{x})$, stresses $\boldsymbol{\sigma}(\boldsymbol{x})$, and considering the macro-scale cohesive surface $\mathcal{S}$, displacement jump and traction across the interfaces are denoted $\boldsymbol{\beta}$ and $\boldsymbol{T}$, respectively.

(ii) Each point $\boldsymbol{x}$ at the macro-scale is linked to an RVE whose domain is denoted $\Omega_{\mu}$. Points in $\Omega_{\mu}$ are identified with $\boldsymbol{y}$. Micro-scale entities are distinguished 


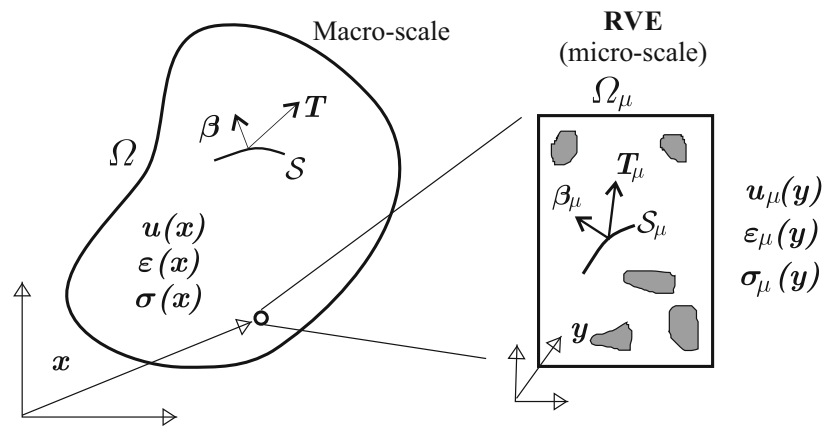

Fig. 1 Notation adopted in this work at the macro and micro-scale levels

with subscript $(\cdot)_{\mu}$, and are understood as different objects to those defined at the macro-scale in the previous item, for example micro-scale displacements, $\boldsymbol{u}_{\mu}$, micro-scale strains, $\boldsymbol{\varepsilon}_{\mu}$, micro-scale stresses, $\boldsymbol{\sigma}_{\mu}$, displacement jumps across micro-scale cracks $\boldsymbol{\beta}_{\mu}$, tractions across micro-scale cohesive cracks $\boldsymbol{T}_{\mu}$, and so on. All micro-scale fields depend on $\boldsymbol{y}$ and $\boldsymbol{x}$.

(iii) Each micro-scale kinematic field has a fluctuating component denoted with a tilde: $(\cdot)_{\mu}$.

(iv) The kinematics at both scales are presented in incremental form. Then, infinitesimal increment of variables are identified with the symbol $d(\cdot)$. Kinematically admissible virtual actions are denoted with the symbol $(\hat{\circ})$.

The structural loading history changes according to a monotonous increase of the pseudo-time coordinate $t$. Then, this pseudo-time is adopted as the parameter indicating the sequence of events taking place in the mechanical problem.

\subsection{Macro-scale model with cohesive surfaces}

Let us consider the domain $\Omega$ of a body displaying an evolving cohesive surface denoted $\mathcal{S}$ whose orthogonal unit vector is $\boldsymbol{n}$, as shown in Fig. 2a. The surface $\mathcal{S}$ shows a relative displacement between both interfaces, also called displacement jump vector, denoted $\boldsymbol{\beta}$, and cohesive tractions $\boldsymbol{T}$ acting on them.

The cohesive surface $\mathcal{S}$ is nucleated at the macro-scale when the material instability condition is detected at a certain pseudo-time instant $\left(t=t_{N}\right)$. The criterion defining $t_{N}$ is presented in Sect. 3.1.1.

The surface $\mathcal{S}$ divides $\Omega$ in two disjoint sets: $\Omega_{+}$and $\Omega_{-}$. On the boundary $\Gamma$ of $\Omega$, there are imposed tractions $\boldsymbol{t}^{e}$, on $\Gamma_{t}$, and displacements $\boldsymbol{u}_{D}$, on $\Gamma_{D}$, being: $\Gamma=\Gamma_{t} \cup \Gamma_{D}$, see Fig. 2a. We also consider an arbitrarily small domain $\Omega^{\varphi} \subset \Omega$ and $\mathcal{S} \subset \Omega^{\varphi}$. Then, $\Omega^{\varphi}$ is also divided by $\mathcal{S}$ in two disjoint sets: $\Omega_{+}^{\varphi}$ and $\Omega_{-}^{\varphi}$.

Following to Simo et al. [45], the displacement increment $d \boldsymbol{u}$ at the macro-scale is described by: $d \boldsymbol{u}=d \overline{\boldsymbol{u}}+\mathscr{M} d \boldsymbol{\beta}$,

where $d \overline{\boldsymbol{u}}$ represents the increments of the continuous displacement components in $\Omega$. The increment of the displacement jump across $\mathcal{S}$ is $d \boldsymbol{\beta}$ and $\mathscr{M}$ is the unit jump function (with compact support) defined as follows:

$\mathscr{M}:=\mathscr{H}-\varphi$,
$\mathscr{H}=\left\{\begin{array}{ll}0 & \forall x \in \Omega_{-} \\ 1 & \forall x \in \Omega_{+},\end{array} \quad \varphi=\left\{\begin{array}{ll}0 & \forall x \in \Omega_{-} \backslash \Omega_{-}^{\varphi} \\ 1 & \forall x \in \Omega_{+} \backslash \Omega_{+}^{\varphi}\end{array}\right.\right.$,

with $\mathscr{H}$ being the Heaviside step function shifted to $\mathcal{S}$, and $\varphi$ satisfies (3), but otherwise it is an arbitrary sufficiently smooth function, see Fig. 2 b.

According to Eq. (1), and following a previous work of the authors [44], it is assumed the existence of a generalized strain increment $d \varepsilon$ in $\Omega$. This strain increment is derived by applying a generalized gradient to the displacement field in Eq. (1) (see for example [45]). Operating in this way, $d \varepsilon$ is given as follows:

$$
\begin{aligned}
d \boldsymbol{\varepsilon} & =\underbrace{\nabla_{x}^{s} d \bar{u}}_{d \boldsymbol{\varepsilon}_{\bar{u}}}+\underbrace{\mathscr{M} \nabla_{x}^{s} d \boldsymbol{\beta}-\nabla_{x}^{s} \varphi \otimes^{s} d \boldsymbol{\beta}}_{d \boldsymbol{\varepsilon}_{\beta}}+\delta_{S}\left(d \boldsymbol{\beta} \otimes^{s} \boldsymbol{n}\right) \\
& =d \varepsilon_{\bar{u}}+d \varepsilon_{\beta}+\delta_{S}\left(d \boldsymbol{\beta} \otimes^{s} \boldsymbol{n}\right) .
\end{aligned}
$$

where it is assumed that $d \boldsymbol{\beta}$ has compact support in $\Omega^{\varphi}$, the symbol $\otimes^{s}$ represents the symmetric tensor product, and the Dirac delta function shifted to $\mathcal{S}$ is denoted $\delta_{S}\left(\delta_{S}\right.$ has units of length ${ }^{-1}$ ).

Remark: the last term in (4) introduces a strain singularity in $\mathcal{S}$. Therefore, it is distinguished between: $a$ ) regular points, denoted $\boldsymbol{x}_{R}\left(\boldsymbol{x}_{R} \in \Omega \backslash \mathcal{S}\right)$, from now on called the bulk material and where strains can be conventionally defined, and $b$ ) singular points denoted $\boldsymbol{x}_{S}\left(\boldsymbol{x}_{S} \in \mathcal{S}\right)$, as shown in Fig. 2c.

The regular terms in (4) are identified with the strain increment $d \varepsilon_{R}$ in the bulk material:

$d \varepsilon_{R}=d \varepsilon_{\bar{u}}(d \overline{\boldsymbol{u}})+d \varepsilon_{\beta}(d \boldsymbol{\beta}), \quad \forall \boldsymbol{x}_{R} \in \Omega \backslash \mathcal{S}$.

The partition of the strain increment $d \varepsilon_{R}$ in two terms, $d \varepsilon_{\bar{u}}$ and $d \varepsilon_{\beta}$ as shown in Eqs. (4) and (5), evidences the fact that $d \varepsilon_{\bar{u}}$ only depends on $d \overline{\boldsymbol{u}}$, while $d \varepsilon_{\beta}$ only depends on $d \beta$. Note that $d \varepsilon_{R}$ in the present kinematical description, even though it is defined in regular points, depends on $d \boldsymbol{\beta}$ due to the compact support of the function $\mathscr{M}$, see (2).

From (4), we also identify the singular strain increment quantity $d \varepsilon_{S}$ in $\mathcal{S}$ as follows:

$d \varepsilon_{S}=d \varepsilon_{\bar{u}}+d \varepsilon_{\beta}+\delta_{S}\left(d \boldsymbol{\beta} \otimes^{S} \boldsymbol{n}\right), \quad \forall \boldsymbol{x}_{S} \in \mathcal{S}$. 


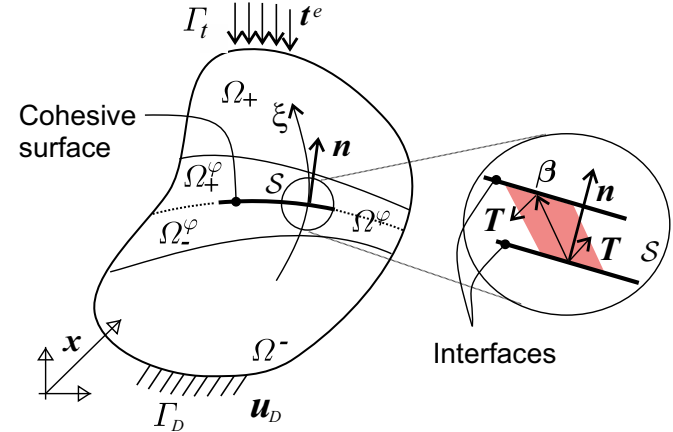

(a)

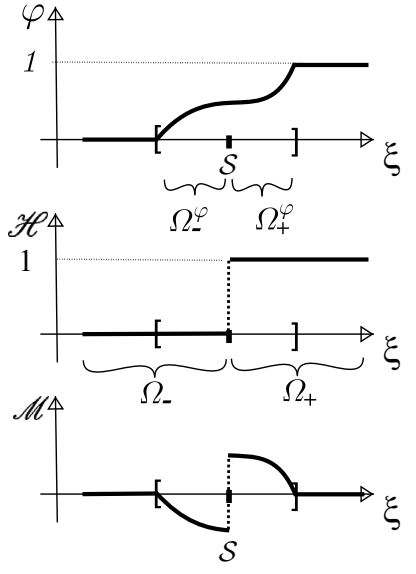

(b)

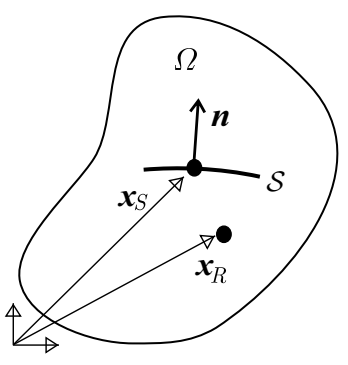

(c)

Fig. 2 Macro-scale model: a cohesive surface at the macro-scale and its mechanical representation; b Continuous function $\varphi$, Heaviside step function $\mathscr{H}$ and unit jump function $\mathscr{M}$; c regular and singular points at the macro-scale

This generalized strain is implicitly represented by the triad of kinematical descriptors $\left\{d \varepsilon_{\bar{u}}, d \varepsilon_{\beta}, d \boldsymbol{\beta}\right\}$. From now on, we describe $d \varepsilon_{S}$ by this implicit representation.

The momentum balance equation in $\Omega$ can be written in a variational format as follows: find $(\overline{\boldsymbol{u}}, \boldsymbol{\beta}) \in \mathscr{U}$ such that:

$$
\begin{aligned}
& \int_{\Omega \backslash \mathcal{S}} \boldsymbol{\sigma} \cdot \hat{\varepsilon}_{R} d \Omega+\int_{\mathcal{S}} \boldsymbol{T} \cdot \hat{\boldsymbol{\beta}} d \mathcal{S} \\
& -\int_{\Gamma_{t}} \boldsymbol{t}^{e} \cdot \hat{\overline{\boldsymbol{u}}} d \Gamma=0, \quad \forall(\hat{\overline{\boldsymbol{u}}}, \hat{\boldsymbol{\beta}}) \in \mathscr{V},
\end{aligned}
$$

where $\mathscr{U}$ is the set of kinematically admissible descriptors and $\mathscr{V}$ the space of kinematically admissible virtual actions given as follows:

$$
\begin{aligned}
& \mathscr{U}:=\left\{(\overline{\boldsymbol{u}}, \boldsymbol{\beta}) ; \quad \overline{\boldsymbol{u}} \in \boldsymbol{H}^{1}(\Omega), \boldsymbol{\beta} \in \boldsymbol{H}^{1}\left(\Omega^{\varphi}\right) \text { and }\left.\overline{\boldsymbol{u}}\right|_{\Gamma_{D}}=\boldsymbol{u}_{D}\right\} \text {, } \\
& \mathscr{V}:=\left\{(\hat{\overline{\boldsymbol{u}}}, \hat{\boldsymbol{\beta}}) ; \hat{\overline{\boldsymbol{u}}} \in \boldsymbol{H}^{1}(\Omega), \hat{\boldsymbol{\beta}} \in \boldsymbol{H}^{1}\left(\Omega^{\varphi}\right) \text { and }\left.\hat{\overline{\boldsymbol{u}}}\right|_{\Gamma_{D}}=\mathbf{0}\right\} .
\end{aligned}
$$

\subsubsection{Multi-scale evaluation of stresses and tractions}

In Eq. (7), stresses $\boldsymbol{\sigma}$ at regular points, $\boldsymbol{x}_{R}$, and tractions $\boldsymbol{T}$ at singular points, $\boldsymbol{x}_{S}$, are evaluated using specific non-standard homogenization procedures gathering information from the micro-scale. These homogenization procedures are rationally justified within the framework of the MMVP theory ([33] and [44]). Each of them is typified as follows:

(i) MMRp (Multi-scale constitutive Model at Regular points): this homogenization procedure defines the overall bulk material response, $\sigma$, at $\boldsymbol{x}_{R}$; (ii) MMSp (Multi-scale constitutive Model at Singular points): this homogenization procedure defines the overall traction-separation response, $\boldsymbol{T}$, at $\boldsymbol{x}_{S}$.

Fig. 3 sketches the big picture of the homogenization procedure in $\Omega$,identifying the MMRp and MMSp procedures and the macro-scale points where each of them is utilized. Every macro-scale point has its own RVE. Incremental strains and generalized incremental strains are inserted from the macroscale to the micro-scale, while stresses and tractions are obtained from the micro-scale, by applying homogenization techniques. The process of inserting strains and retrieving generalized stresses is governed by a set of scale bridging equations which are defined and described in detail in Sect. 2.3.

\subsection{Micro-scale model with cohesive surfaces}

The kinematical description at the RVE is similar to that utilized at the macro-scale. So, the failure mechanism is described by means of cohesive surfaces which are nucleated as the structural loading increases.

Let us consider the RVE domain $\Omega_{\mu}$ displaying $n_{c}$ cohesive surfaces nucleated at the micro-scale during the loading process. The $i$-th cohesive surface is denoted $\mathcal{S}_{\mu}^{i}$, and its normal vector is $\boldsymbol{n}_{\mu}^{i}$, see Fig. 4a. The cohesive surface $\mathcal{S}_{\mu}^{i}$ divides $\Omega_{\mu}$ in two disjoint domains, $\Omega_{\mu_{+}}^{i}$ and $\Omega_{\mu_{-}}^{i}$, with $\boldsymbol{n}_{\mu}^{i}$ pointing toward $\Omega_{\mu_{+}}$.

A micro-scale cohesive crack $\mathcal{S}_{\mu}^{i}$ nucleates when the material bifurcation condition is satisfied at a given point of the micro-scale (at pseudo-time $t_{N_{\mu}}$ ), and previously to reaching the critical condition at the macro-scale for crack nucleation. 


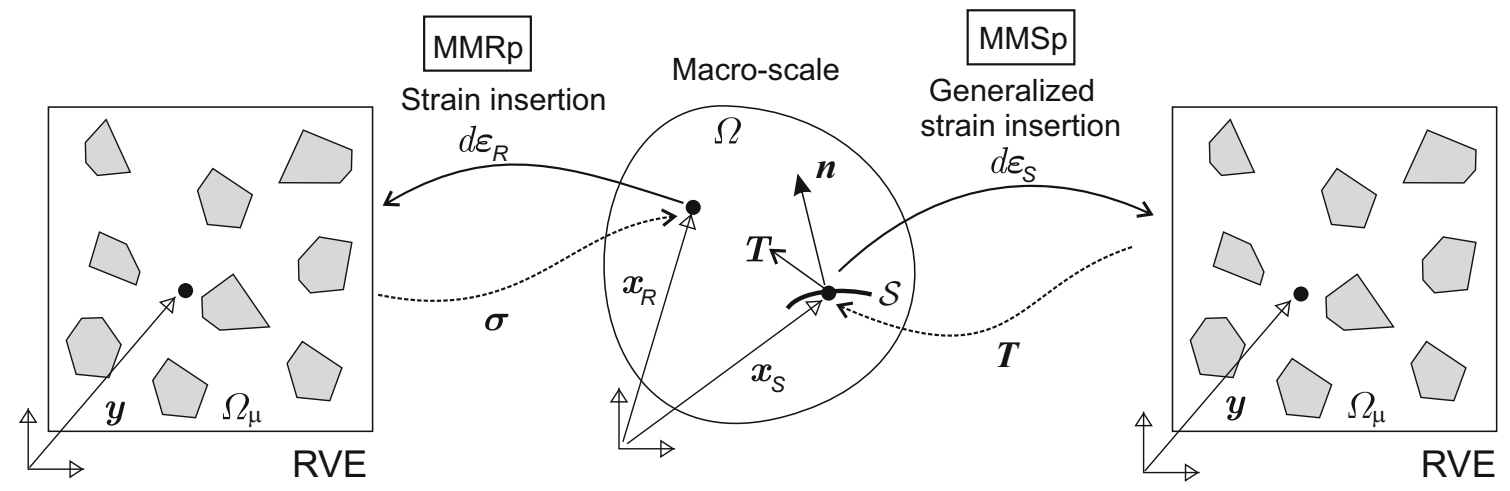

Fig. 3 Multi-scale model for fracture. The MMRp homogenization procedure evaluates the overall material response, $\boldsymbol{\sigma}$, at regular points $\boldsymbol{x}_{R}$. The MMSp homogenization procedure evaluates the overall material response, $\boldsymbol{T}$, on the cohesive surface, at points $\boldsymbol{x}_{S}$

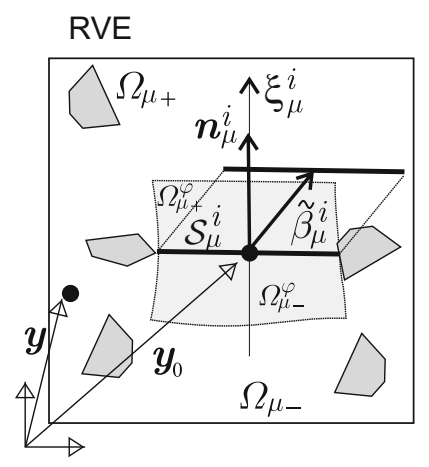

(a)

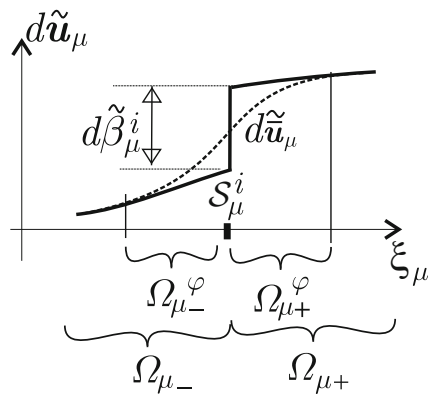

(b)

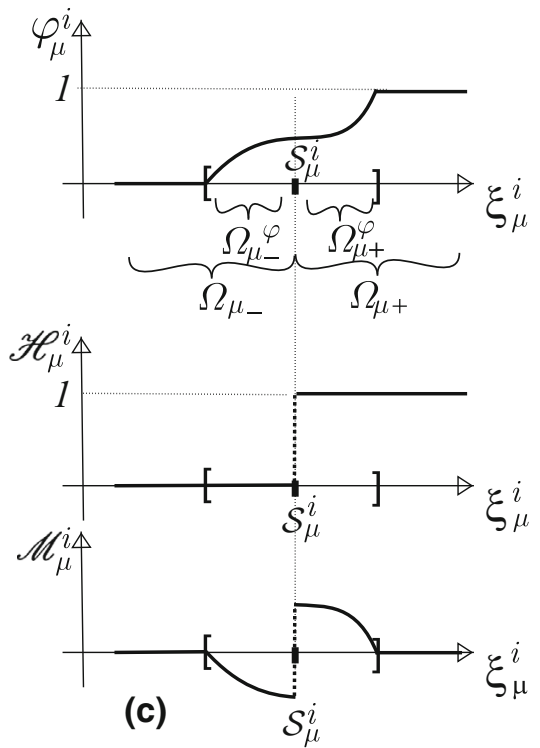

Fig. 4 Kinematics at the micro-scale accounting for material failure. a cohesive surface $\mathcal{S}_{\mu}^{i}$; b micro-scale displacement fluctuation increment field, $d \tilde{\boldsymbol{u}}_{\mu}$, displaying a strong discontinuity across $\mathcal{S}_{\mu}^{i} ; \mathbf{c}$ continuous function $\varphi_{\mu}^{i}$, Heaviside function $\mathscr{H}_{\mu}^{i}$ and unit jump function $\mathscr{M}_{\mu}^{i}$

The micro-scale strain increment $d \varepsilon_{\mu}$ in $\Omega_{\mu}$ is:

$d \varepsilon_{\mu}=\mathcal{I}(d \varepsilon)+d \tilde{\varepsilon}_{\mu}$,

where the first term corresponds to the macro-scale strain increment, $d \varepsilon$, inserted from $\boldsymbol{x}$ into $\Omega_{\mu}$, through the insertion operator $\mathcal{I}(\cdot)$. This insertion operator $\mathcal{I}(\cdot)$ distributes its argument into the RVE domain. Both, $\mathcal{I}$ as well as its argument, changes according with the homogenization procedure. The operator $\mathcal{I}$ is defined in next Sect. 2.3 where it is labeled $\mathcal{I}_{R}$ or $\mathcal{I}_{S}$ according to the MMRs or MMSp procedure, respectively.

The last term on the right hand side of (9) is the microscale strain fluctuation increment, defined as:

$d \tilde{\varepsilon}_{\mu}=\nabla_{y}^{s} d \tilde{\boldsymbol{u}}_{\mu}$ where $d \tilde{\boldsymbol{u}}_{\mu}$ is the micro-scale displacement fluctuation increment, whose description is discussed in the following.

\subsubsection{Treatment given to the micro-scale displacement fluctuation increment field}

Similar to the strong discontinuity kinematics adopted at the macro-scale, described by Eq. (1), the micro-scale displacement fluctuation increment, $d \tilde{\boldsymbol{u}}_{\mu}$, is written as the addition of two terms, see Fig. 4b:

$d \tilde{\boldsymbol{u}}_{\mu}=d \tilde{\overline{\boldsymbol{u}}}_{\mu}+\sum_{i=1}^{n_{c}} \mathscr{M}_{\mu}^{i} d \tilde{\boldsymbol{\beta}}_{\mu}^{i}$,

where $\tilde{\overline{\boldsymbol{u}}}_{\mu}$ is the continuous component of the micro-scale displacement fluctuation, $\tilde{\boldsymbol{\beta}}_{\mu}^{i}$ is the displacement jump fluc- 
tuation across the interfaces of the $i$-th cohesive surface $\mathcal{S}_{\mu}^{i}$ and $\mathscr{M}_{\mu}^{i}$ is the unit jump function. Both, $\tilde{\boldsymbol{\beta}}_{\mu}^{i}$ and $\mathscr{M}_{\mu}^{i}$, are related to $\mathcal{S}_{\mu}^{i}$. In (11), we assume that the field $d \tilde{\boldsymbol{u}}_{\mu}$ results from the addition of all the contributions of the $n_{c}$ cohesive surfaces which have nucleated during the loading history. The function $\mathscr{M}_{\mu}^{i}$ is defined as follows, see Fig. 4c:

$$
\begin{aligned}
& \mathscr{M}_{\mu}^{i}:=\mathscr{H}_{\mu}^{i}-\varphi_{\mu}^{i}, \\
& \mathscr{H}_{\mu}^{i}=\left\{\begin{array}{ll}
0 & \forall \boldsymbol{y} \in \Omega_{\mu_{-}}^{i} \\
1 & \forall \boldsymbol{y} \in \Omega_{\mu_{+}}^{i}
\end{array}, \quad \varphi_{\mu}^{i}= \begin{cases}0 & \forall \boldsymbol{y} \in \Omega_{\mu_{-}}^{i} \backslash \Omega_{\mu}^{i \varphi} \\
1 & \forall \boldsymbol{y} \in \Omega_{\mu_{+}}^{i} \backslash \Omega_{\mu_{+}}^{i \varphi},\end{cases} \right.
\end{aligned}
$$

where the Heaviside step function $\mathscr{H}_{\mu}^{i}$ and the smooth function $\varphi_{\mu}^{i}$ are also related to the same $i$-th cohesive crack. In this case, $\Omega_{\mu}^{i \varphi}$ is a small domain around $\mathcal{S}_{\mu}^{i}$ (with: $\mathcal{S}_{\mu}^{i} \subset$ $\Omega_{\mu}^{i \varphi} \subset \Omega_{\mu}$ ), while $\Omega_{\mu}^{i \varphi}$ and $\Omega_{\mu}^{i \varphi}$ are subdomains of $\Omega_{\mu}^{i \varphi}$ partitioned by $\mathcal{S}_{\mu}^{i}$, as shown in Fig. 4a.

According with (11), the micro-scale strain fluctuation increment, $d \tilde{\varepsilon}_{\mu}$, is given by:

$$
d \tilde{\varepsilon}_{\mu}=d \tilde{\varepsilon}_{\mu_{R}}+\sum_{i=1}^{n_{c}} \delta_{\mathcal{S}_{\mu}^{i}}\left(d \tilde{\boldsymbol{\beta}}_{\mu}^{i} \otimes^{s} \boldsymbol{n}_{\mu}^{i}\right), \quad \forall \boldsymbol{y} \in \Omega_{\mu},
$$

where the first (regular) term on the right hand side is:

$$
d \tilde{\boldsymbol{\varepsilon}}_{\mu_{R}}=\nabla_{y}^{s} d \tilde{\overline{\boldsymbol{u}}}_{\mu}+\sum_{i=1}^{n_{c}}\left(\mathscr{M}_{\mu}^{i} \nabla_{y}^{s} d \tilde{\boldsymbol{\beta}}_{\mu}^{i}-\nabla_{y}^{s} \varphi_{\mu}^{i} \otimes^{s} d \tilde{\boldsymbol{\beta}}_{\mu}^{i}\right)
$$

and the second (singular) term is defined by the Dirac delta function $\delta_{\mathcal{S}_{\mu}^{i}}$ shifted to $\mathcal{S}_{\mu}^{i}$.

\subsection{Scale-bridging equations}

In the present sub-Section, the scale bridging equations of the multi-scale model presented in [44] are summarized. The readers are addressed to that reference to find the full description of the concepts supporting these equations.

The scale bridging equations are established from the following two axioms and the consequences derived from them, see further details in [33]:

H1 Kinematical admissibility requirements connecting the kinematics at macro and micro-scales. This hypothesis imposes the following constraints on the micro-scale strains:

$$
\int_{\Omega_{\mu}^{*}} d \varepsilon_{\mu} d \Omega_{\mu}=\int_{\Omega_{\mu}^{*}} \mathcal{I}(d \varepsilon) d \Omega_{\mu}
$$

The insertion operator $\mathcal{I}$, its arguments and the microscale domain $\Omega_{\mu}^{*}$ where identity (16) is imposed, depend on the homogenization procedure, MMRp or MMSp (see Eq. (18), (29) and (30) in the following paragraphs).

From Eq. (9), condition (16) enforces a null average of the micro-scale strain fluctuation, $d \tilde{\varepsilon}_{\mu}$, in $\Omega_{\mu}^{*}$. This kinematical admissibility requirement is conventional in semi-concurrent multi-scale models using a RVE (where $\Omega_{\mu}^{*} \equiv \Omega_{\mu}$, and $\mathcal{I}(d \varepsilon)$ is the identity operator). However, it has been generalized in [44] in order to develop a consistent homogenization model for the singular points $\boldsymbol{x}_{S}$.

H2 Identity, in a variational sense, of internal virtual power densities between both scales. This hypothesis is the Principle of Multi-scale Virtual Power, according with [33], and is a generalized version of the Hill-Mandel Principle. Hypothesis (H2) has been specified in [44] to include the presence of strong discontinuities at both scales of analysis.

As mentioned above, at the macro-scale level, the multiscale model distinguishes between regular points, $\boldsymbol{x}_{R}$, and singular points, $\boldsymbol{x}_{S}$. Different strain insertion procedures are defined in those points (MMRp and MMSp). Then, hypotheses $\mathrm{H} 1$ and $\mathrm{H} 2$ should be specified in each case (Fig. 5).

As a realistic assumption for modeling multi-scale material failure problems, it is considered that during the macroscale pre-bifurcation regime at a given point $\boldsymbol{x}_{R}$, there have been nucleated $n_{c}$ micro-scale cohesive cracks in $\Omega_{\mu}$. These micro-cracks are denoted $\mathcal{S}_{\mu}^{i}$ (with $i=1, \ldots, n_{c}$ ). The number $n_{c}$ changes in the loading history because new cohesive micro-scale cracks may sequentially nucleate during $t<t_{N}$.

However, when bifurcation at the macro-scale is detected, at $t=t_{N}$, from the $n_{c}$ nucleated micro-scale cohesive cracks in $\Omega_{\mu}, n_{o p}$ cracks are opening and $n_{c l}$ are closing or remain inactive $\left(n_{c}=n_{c l}+n_{o p}\right) . \mathcal{S}_{\mu}^{L_{j}}$ (with $\left.j=1, \ldots, n_{o p}\right)$ denotes the $j$-th opening crack, and $\mathcal{S}_{\mu}^{L}$ is the set of all the opening cracks $\left(\mathcal{S}_{\mu}^{L}=\cup_{j=1}^{n_{o p}} \mathcal{S}_{\mu}^{L j}\right)$. There are not new micro-scale cracks which can nucleate or re-open during the subsequent loading history. So, the micro-scale failure mode is defined by the set of the $n_{o p}$ opening cracks at $t=t_{N}$, and after that, it does not change anymore.

In order to apply the scale transition equations of the MMSp homogenization procedure, the domain $\mathcal{S}_{\mu}^{L}$ has to be precisely detected at $t=t_{N}$. The algorithm to define $\mathcal{S}_{\mu}^{L}$ is described in the numerical implementation Section.

Taking into account the previous concepts, it is defined a tortuosity factor $\theta$, as follows: 


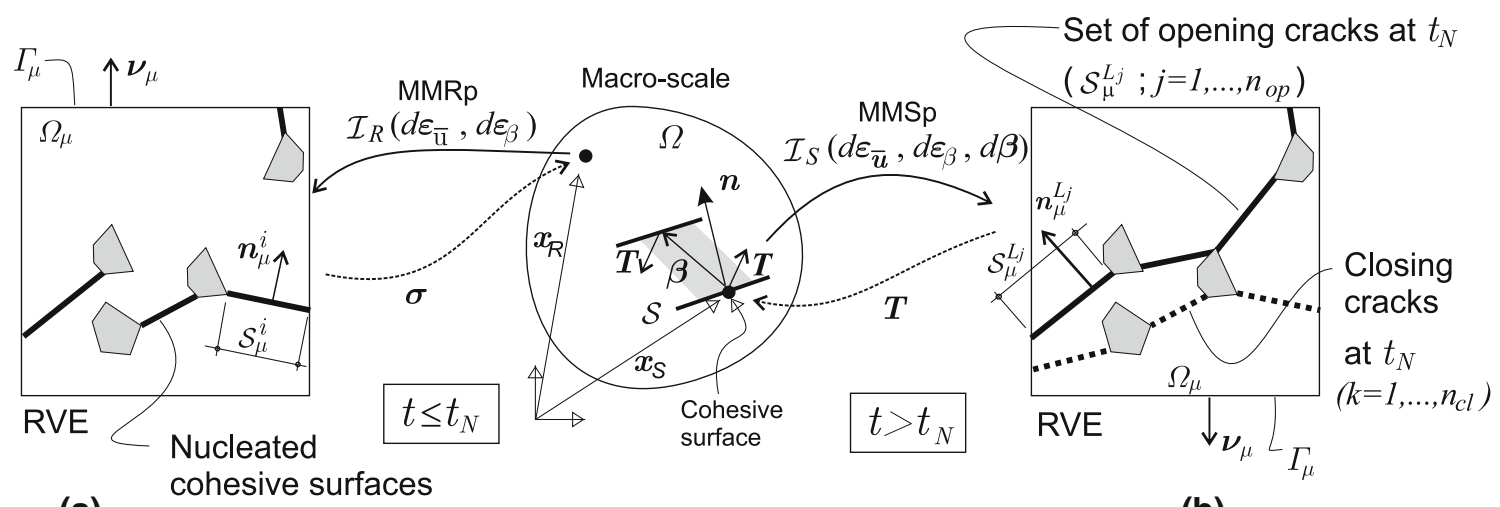

(a) $\quad\left(i=1, \ldots, n_{c}\right)$

(b)

Fig. 5 Overview of the multi-scale model for fracture: a the MMRp homogenization procedure uses the insertion operator $\mathcal{I}_{R}$ which is defined in Box 1; $\mathbf{b}$ the MMSp homogenization procedure uses the insertion operator $\mathcal{I}_{S}$ which is defined in Box 2

$$
\theta\left(\boldsymbol{x}_{S}\right)=\frac{\left|\mathcal{S}_{\mu}^{L}\right|}{\left\|\left(\sum_{j=1}^{n_{o p}} \int_{\mathcal{S}_{\mu}^{L_{j}}} \boldsymbol{n}_{\mu}^{L_{j}} d \mathcal{S}_{\mu}\right)\right\|}, \quad\left|\mathcal{S}_{\mu}^{L}\right|=\sum_{j=1}^{n_{o p}}\left|\mathcal{S}_{\mu}^{L_{j}}\right|,
$$

where $\left|\mathcal{S}_{\mu}^{L_{j}}\right|$ is the measure of the cohesive surface $\mathcal{S}_{\mu}^{L_{j}}$.

The parameter $\theta \in[1, \infty)$ is interpreted as a geometrical factor measuring the tortuosity of the micro-scale crack path of the failure mechanism at the RVE, at $t=t_{N}$. It is introduced to guarantee the kinematical consistency associated with the cracks opening at both scales of analysis: the opening of the macro-crack $\mathcal{S}$ and the openings, in several directions, of the multiple cracks simulated at the micro scale level. Note that $\theta=1$ if all the micro-scale cracks are parallel, and it is larger than 1 if the micro-scale cracks follow a zig-zag path. A sensitivity analysis of the macro-scale behavior with respect to $\theta$ has been presented in a previous work of the authors, see Toro et al. [44]. In such contribution, a rigorous kinematical justification for including the $\theta$-factor in the multi-scale model has also been discussed.

The tortuosity parameter $\theta$ scales all terms involving the macro-scale displacement jump $\boldsymbol{\beta}$ intervening in the scale transition procedure.

The scale bridging equations governing the multi-scale problem are summarized in Boxes 1 and 2. However, before discussing these equations, in Fig. 6 we sketch, by means of three blocks, the sequence of macro-scale kinematical, constitutive and variational equilibrium equations that govern a mechanical problem within the framework of the present multi-scale approach. These three blocks are contained into a larger box identified as "Macro-scale Mechanical Problem", that represents the iterative strategy (for example the Newton-Raphson scheme), for solving the mechanical problem. This Figure also describes the information flow across the three blocks and the corresponding equations which characterize each block. Specifically, the block representing the macro-scale constitutive model is divided in two subblocks according if the constitutive model refers to a regular $\left(x_{R}\right)$ or a singular $\left(x_{\mathcal{S}}\right)$ point, and therefore to the MMRp or MMSp homogenization procedure. The RVE problems of both homogenization procedures are sketched in detail in Fig. 7, which follows a similar organization used in Fig. 6.

The equations summarized in Boxes 1 and 2 are related to the sub-block "Macro-Scale Constitutive Models" in Fig. 6. They are explained in the next two items:

\section{(i) MMRp homogenization procedure to obtain $\sigma$ in $x_{R}$} (see Box 1).

The regular macro-scale strain increment, $d \varepsilon_{R}$, is the data input of the multi-scale constitutive relation described in Box 1. According to Eq. (5), $d \varepsilon_{R}$ is given by the addition of two components: $d \varepsilon_{\bar{u}}$ and $d \varepsilon_{\beta}$.

The first block of Eqs. (20)-(26) in Box 1, defines the micro-scale kinematics in $\Omega_{\mu}$ and the functional spaces involved in the variational formulation of the micro-scale problem.

The strain increment $d \varepsilon_{\mu}$, at the RVE, is given by Eqs. (20)-(22) and is a particularization of (9). The strain increment $d \varepsilon_{\mu}$ results from the addition of two terms: the macro-scale strain increment $d \varepsilon_{R}$, inserted from the macro-scale into the RVE through the operator $\mathcal{I}_{R}(\cdot)$, plus the strain fluctuation increments $d \tilde{\varepsilon}_{\mu}$. According with the definition of the operator $\mathcal{I}_{R}(\cdot)$, in Eq. (21), both terms $d \varepsilon_{\bar{u}}$ and $d \varepsilon_{\beta}$ are uniformly distributed into $\Omega_{\mu}$. However, note that $d \varepsilon_{\beta}$ is scaled by the tortuosity factor $\theta$.

The Kinematical admissibility requirement (16), hypothesis H1, is particularized as follows:

$\int_{\Omega_{\mu}} d \varepsilon_{\mu} d \Omega_{\mu}=\int_{\Omega_{\mu}} \mathcal{I}_{R}\left(d \varepsilon_{\bar{u}}, d \varepsilon_{\beta}\right) d \Omega_{\mu}$ 
Macro-scale Mechanical Problem

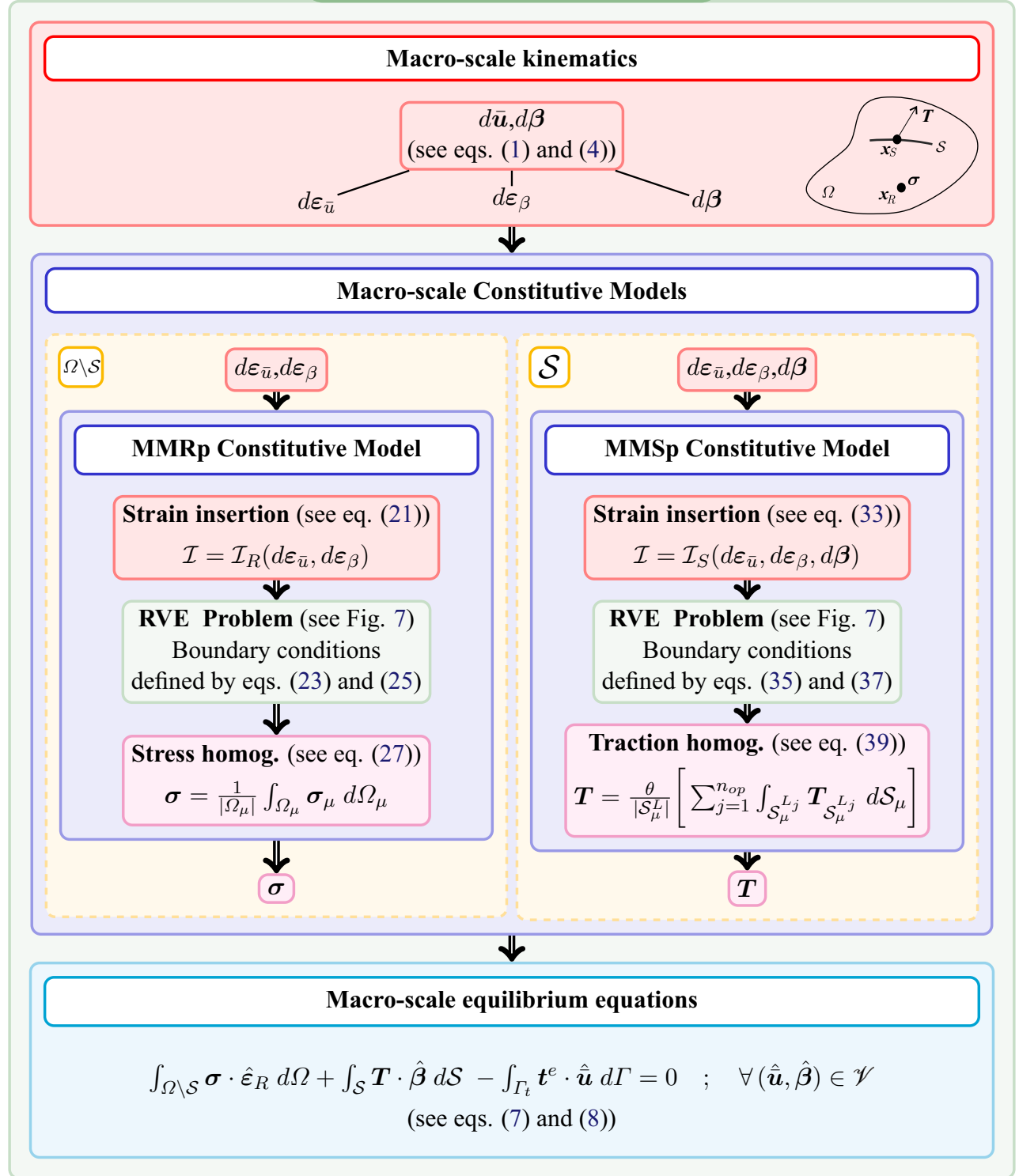

Fig. 6 Data flow of the mechanical problem involving both scales of analysis: macro and micro

see [44] for additional details. Then, expression (23) of Box 1 defines the space $\tilde{\mathscr{U}}_{\mu}^{R}$ of kinematically admissible displacement fluctuations. As mentioned above, the kinematical descriptors fully defining the displacement fluctuations at the micro-scale are: $d \tilde{\overline{\boldsymbol{u}}}_{\mu}$ and $d \tilde{\boldsymbol{\beta}}_{\mu}^{i}$ $\left(i=1, \ldots, n_{c}\right)$. They are assumed to be independent descriptors. Geometrical entities in expression (23), i.e. the boundary $\Gamma_{\mu}$ of $\Omega_{\mu}$ and its unit normal vector $\nu_{\mu}$, are depicted in Fig. 5. The integral restriction on the boundary $\Gamma_{\mu}$ imposed to the continuous displacement fluctuation increment fields in $\tilde{\mathscr{U}}_{\mu}^{R}$, as expressed in (23), guaranties the fulfillment of restriction (18).
Expression (24) defines the virtual actions at the macroscale. Note that in the MMRp model we consider that $\hat{\varepsilon}_{\bar{u}}$ is an arbitrary tensor while $\hat{\boldsymbol{\beta}} \equiv \boldsymbol{O}$. The corresponding space of virtual displacement actions at the micro-scale is $\mathscr{V}_{\mu}^{R}$, and the micro-scale virtual strain actions are defined in expression (26).

Any subspace of $\tilde{\mathscr{U}}_{\mu}^{R}$ and $\mathscr{V}_{\mu}^{R}$, is also kinematically admissible and defines alternative (more constrained) sub-models of the MMRp procedure.

The specific definition of the virtual actions is the basis to postulate the Principle of Multi-scale Virtual Power $(\mathrm{H} 2)$, which is expressed as follows: 


\section{RVE Mechanical Problem}

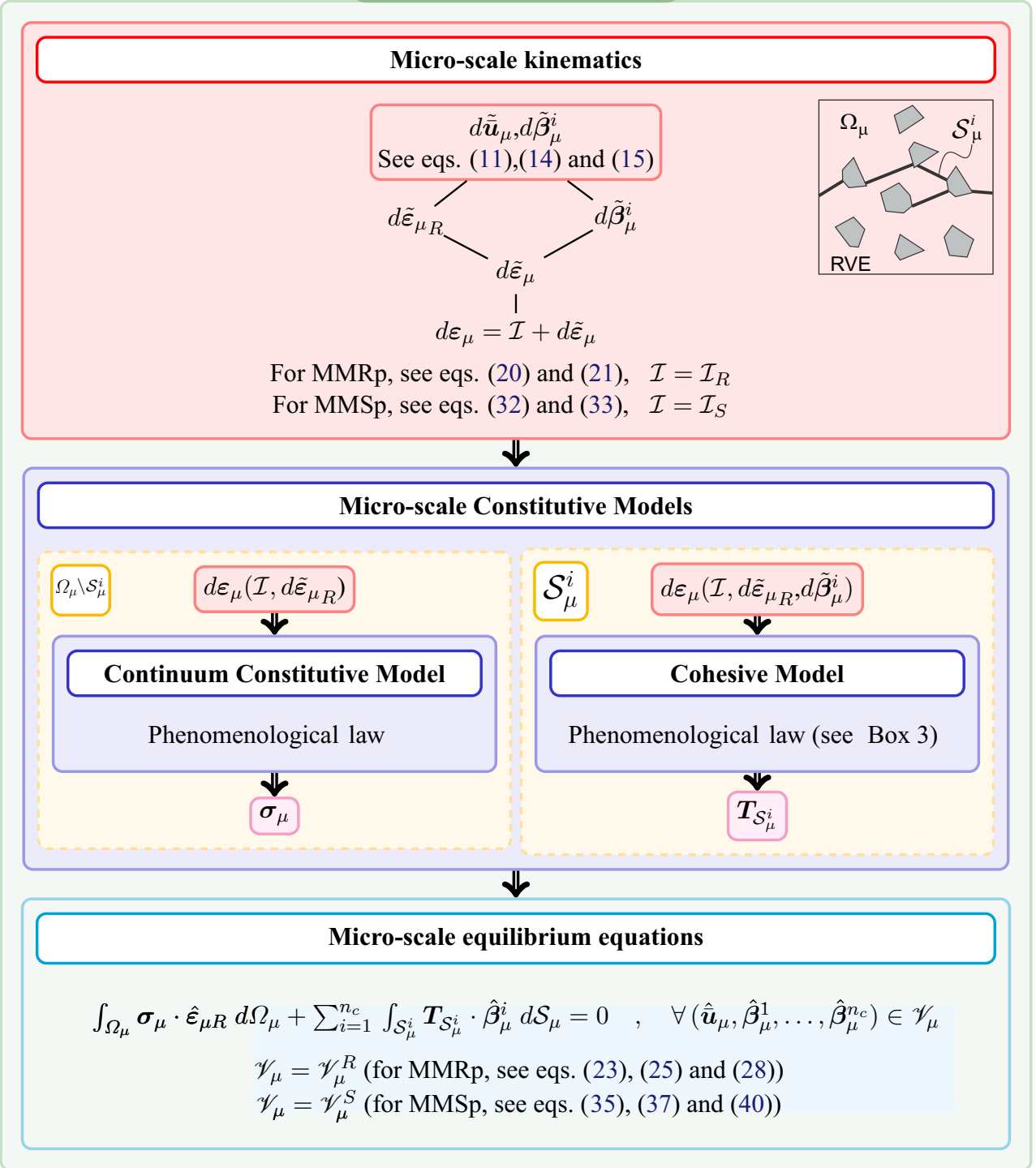

Fig. 7 Data flow of the RVE problem for both homogenization procedures: MMRp and MMSp

$$
\begin{array}{r}
\boldsymbol{\sigma} \cdot \hat{\varepsilon}_{\bar{u}}=\frac{1}{\left|\Omega_{\mu}\right|} \int_{\Omega_{\mu}} \boldsymbol{\sigma}_{\mu} \cdot \hat{\varepsilon}_{\mu}\left(\hat{\varepsilon}_{\bar{u}}, \hat{\overline{\boldsymbol{u}}}_{\mu}, \hat{\boldsymbol{\beta}}_{\mu}^{1}, \ldots, \hat{\boldsymbol{\beta}}_{\mu}^{n_{c}}\right) d \Omega_{\mu}, \\
\forall \hat{\varepsilon}_{\bar{u}} \quad \text { and } \quad \forall\left(\hat{\overline{\boldsymbol{u}}}_{\mu}, \hat{\boldsymbol{\beta}}_{\mu}^{1}, \ldots, \hat{\boldsymbol{\beta}}_{\mu}^{n_{c}}\right) \in \mathscr{V}_{\mu}^{R} .
\end{array}
$$

The left hand side of this equation is the macro-scale internal virtual power density and the right hand side is the average internal virtual power in $\Omega_{\mu}$. Equation (19) emphasizes the dependence of the micro-scale strain variations $\hat{\varepsilon}_{\mu}$ with: $\hat{\varepsilon}_{\bar{u}}, \hat{\overline{\boldsymbol{u}}}_{\mu}, \hat{\boldsymbol{\beta}}_{\mu}^{1}, \ldots, \hat{\boldsymbol{\beta}}_{\mu}^{n_{c}}$. This dependence is explicitly shown in Eq. (26).

After replacing (24) and (26) into (19), the Principle of Multi-scale Virtual Power (H2) implies two natural consequences in the sense of the associated Euler-Lagrange equations. They are derived by considering independent variations of $\hat{\varepsilon}_{\bar{u}}$ and $\left(\hat{\overline{\boldsymbol{u}}}_{\mu}, \hat{\boldsymbol{\beta}}_{\mu}^{1}, \ldots, \hat{\boldsymbol{\beta}}_{\mu}^{n_{c}}\right)$. Both consequences are presented in blocks 2.a and 2.b of Box 1, and are explained as follows:

(2.a) Eq. (27) is derived from (19) by allowing arbitrary variations $\hat{\varepsilon}_{\bar{u}}$ and holding $\left(\hat{\overline{\boldsymbol{u}}}_{\mu}, \hat{\boldsymbol{\beta}}_{\mu}^{1}, \ldots, \hat{\boldsymbol{\beta}}_{\mu}^{n_{c}}\right)$ fixed to zero. This equation defines the homogenization procedure determining the macro-scale stresses $\sigma$, as the volumetric averaging of the micro-scale stress field $\sigma_{\mu}$. The micro-scale stress $\sigma_{\mu}$ is determined from the micro-scale equilibrium problem given by expression (28).

(2.b) Expression (28) is derived from (19) by introducing arbitrary variations of $\left(\hat{\overline{\boldsymbol{u}}}_{\mu}, \hat{\boldsymbol{\beta}}_{\mu}^{1}, \ldots, \hat{\boldsymbol{\beta}}_{\mu}^{n_{c}}\right)$ and holding 
$\hat{\varepsilon}_{\bar{u}}$ fixed to zero. This equation is the variational equilibrium in $\Omega_{\mu}$.

The constitutive relation of every constituent of the heterogeneous material and the traction-separation law of every cohesive surface in $\Omega_{\mu}$ must be specified to solve the microscale equilibrium problem (28), and so, to determine $\sigma_{\mu}$ and the cohesive traction $\boldsymbol{T}_{\mathcal{S}_{\mu}^{i}}$ acting on the micro-scale crack $\mathcal{S}_{\mu}^{i}$. displacement jump $\boldsymbol{\beta}$ (a vector term) is used in Box 2 instead of the singular tensor term described in the right hand side of equation (6).

The first block of Eqs. (32)-(38) in Box 2, defines the micro-scale kinematics in $\Omega_{\mu}$. Following the same notation as above, the micro-scale strain increment is identified as $d \varepsilon_{\mu}$. Also, the micro-scale strain increment fluctuation is denoted $d \tilde{\varepsilon}_{\mu}$, and is defined in Eq. (34). The insertion operator $\mathcal{I}_{S}(\cdot)$, defined in Eq. (33), dis-

\section{Box 1: Multi-scale constitutive Model at Regular points (MMRp)}

Given the strain history: $\varepsilon_{R}^{(t-d t)}$ and $\left\{d \varepsilon_{\bar{u}}, d \varepsilon_{\beta}\right\}$, find $\sigma$ satisfying :

1- Kinematics:

$d \boldsymbol{\varepsilon}_{\mu}=\mathcal{I}_{R}\left(d \varepsilon_{\bar{u}}, d \boldsymbol{\varepsilon}_{\beta}\right)+d \tilde{\boldsymbol{\varepsilon}}_{\mu} \quad, \quad \forall \boldsymbol{y} \in \Omega_{\mu}$

$\mathcal{I}_{R}\left(d \varepsilon_{\bar{u}}, d \varepsilon_{\beta}\right)=d \varepsilon_{\bar{u}}+\theta d \varepsilon_{\beta}$

$d \tilde{\boldsymbol{\varepsilon}}_{\mu}=\nabla_{y}^{s} d \tilde{\overline{\boldsymbol{u}}}_{\mu}+\sum_{i=1}^{n_{c}}\left(\mathscr{M}_{\mu}^{i} \nabla_{y}^{s} d \tilde{\boldsymbol{\beta}}_{\mu}^{i}-\nabla_{y} \varphi_{\mu}^{i} \otimes^{s} d \tilde{\boldsymbol{\beta}}_{\mu}^{i}+\delta_{\mathcal{S}_{\mu}^{i}}\left(d \tilde{\boldsymbol{\beta}}_{\mu}^{i} \otimes^{s} \boldsymbol{n}_{\mu}^{i}\right)\right)$

$d \boldsymbol{\beta}_{\mu}^{i}=d \tilde{\boldsymbol{\beta}}_{\mu}^{i} ;$ for: $i=1, \ldots, n_{c}$

Kinematically admissible space of incremental displacement fluctuations, $d \tilde{\boldsymbol{u}}_{\mu}$ :

$$
\tilde{\mathscr{U}}_{\mu}^{R}=\left\{\left(d \tilde{\overline{\boldsymbol{u}}}_{\mu}, d \tilde{\boldsymbol{\beta}}_{\mu}^{1}, \ldots, d \tilde{\boldsymbol{\beta}}_{\mu}^{n_{c}}\right) \text { suff. regular, such that: } \int_{\Gamma_{\mu}} d \tilde{\overline{\boldsymbol{u}}}_{\mu} \otimes^{s} \boldsymbol{v}_{\mu} d \Gamma_{\mu}=\mathbf{0}\right\}
$$

Virtual kinematically admissible actions:

Virtual actions at the macro-scale:

$$
\hat{\boldsymbol{\beta}}=\mathbf{0} \quad, \quad \hat{\varepsilon}_{\bar{u}} \text { arbitrary }
$$

Virtual actions at the micro-scale:

$$
\begin{aligned}
& \left(\hat{\overline{\boldsymbol{u}}}_{\mu}, \hat{\boldsymbol{\beta}}_{\mu}^{1}, \ldots, \hat{\boldsymbol{\beta}}_{\mu}^{n_{c}}\right) \in \mathscr{V}_{\mu}^{R} \quad, \quad \text { with: } \quad \mathscr{V}_{\mu}^{R} \equiv \tilde{\mathscr{U}}_{\mu}^{R} \\
& \hat{\boldsymbol{\varepsilon}}_{\mu}=\mathcal{I}_{R}\left(\hat{\boldsymbol{\varepsilon}}_{\bar{u}}, \boldsymbol{0}\right)+\nabla_{y}^{s} \hat{\overline{\boldsymbol{u}}}_{\mu}+\sum_{i=1}^{n_{c}}\left(\mathscr{M}_{\mu}^{i} \nabla_{y}^{s} \hat{\boldsymbol{\beta}}_{\mu}^{i}-\nabla_{y} \varphi_{\mu}^{i} \otimes^{s} \hat{\boldsymbol{\beta}}_{\mu}^{i}+\delta_{\mathcal{S}_{\mu}^{i}}\left(\hat{\boldsymbol{\beta}}_{\mu}^{i} \otimes^{s} \boldsymbol{n}_{\mu}^{i}\right)\right)
\end{aligned}
$$

2-Consequences derived from the Principle of Multi-scale Virtual Power

\section{2.a Stress Homogenization:}

$$
\sigma=\frac{1}{\left|\Omega_{\mu}\right|} \int_{\Omega_{\mu}} \sigma_{\mu} d \Omega_{\mu}
$$

2.b Micro-scale Equilibrium problem:

Given the strain history $\boldsymbol{\varepsilon}_{R}^{(t-d t)}$ and $\left\{d \boldsymbol{\varepsilon}_{R}\right\}$, find $\left(d \tilde{\overline{\boldsymbol{u}}}_{\mu}, d \tilde{\boldsymbol{\beta}}_{\mu}^{1}, \ldots, d \tilde{\boldsymbol{\beta}}_{\mu}^{n_{c}}\right) \in \tilde{\mathscr{U}}_{\mu}^{R}$ such that:

$$
\int_{\Omega_{\mu}} \boldsymbol{\sigma}_{\mu} \cdot \hat{\boldsymbol{\varepsilon}}_{\mu R} d \Omega_{\mu}+\sum_{i=1}^{n_{c}} \int_{\mathcal{S}_{\mu}^{i}} \boldsymbol{T}_{\mathcal{S}_{\mu}^{i}} \cdot \hat{\boldsymbol{\beta}}_{\mu}^{i} d \mathcal{S}_{\mu}=0 \quad, \quad \forall\left(\hat{\overline{\boldsymbol{u}}}_{\mu}, \hat{\boldsymbol{\beta}}_{\mu}^{1}, \ldots, \hat{\boldsymbol{\beta}}_{\mu}^{n_{c}}\right) \in \mathscr{V}_{\mu}^{R}
$$

(ii) MMSp homogenization procedure to obtain $T$ in $x_{S}$ (see Box 2).

The macro-scale strain increment $d \varepsilon_{S}$, in $\mathcal{S}$, is the data input of the constitutive model in Box 2. This generalized strain increment is defined in Eq. (6) and can be represented by the triad $\left\{d \varepsilon_{\bar{u}}, d \varepsilon_{\beta}, d \boldsymbol{\beta}\right\}$. Note that the tributes the macro-scale strain increments given by $\left\{d \varepsilon_{\bar{u}}, d \varepsilon_{\beta}, d \boldsymbol{\beta}\right\}$ into $\Omega_{\mu}$. Similar to the previous case, the increment of the macro-scale regular strain, $d \varepsilon_{\bar{u}}+$ $\theta d \varepsilon_{\beta}$, is uniformly distributed in $\Omega_{\mu}$. However, $\theta d \boldsymbol{\beta}$ is only distributed into the micro-scale strain localization 
domain $\mathcal{S}_{\mu}^{L}$ corresponding to the $n_{o p}$ opening cohesive surfaces. Therefore, in this case, the generalized strain descriptors $\left\{d \varepsilon_{\bar{u}}, d \varepsilon_{\beta}, d \boldsymbol{\beta}\right\}$, are not uniformly distributed into $\Omega_{\mu}$. Again, in this case, the parameter $\theta$ scales all the terms where the macro-scale displacement jump $\boldsymbol{\beta}$ is present.

In correspondence with (14) and (15), Eq. (34) defines the micro-scale strain increment fluctuation. Note, in this expression, that all the micro-scale nucleated cohesive surfaces contribute to $d \tilde{\varepsilon}_{\mu}$.

The Kinematical admissibility requirement (16), hypothesis H1, is imposed through two equations:

$\int_{\Omega_{\mu}} d \varepsilon_{\mu} d \Omega_{\mu}=\int_{\Omega_{\mu}} \mathcal{I}_{S}\left(d \varepsilon_{\bar{u}}, d \varepsilon_{\beta}, \boldsymbol{O}\right) d \Omega_{\mu}$

$\int_{\mathcal{S}_{\mu}^{L}} d \varepsilon_{\mu} d \Omega_{\mu}=\int_{\mathcal{S}_{\mu}^{L}} \mathcal{I}_{S}\left(d \varepsilon_{\bar{u}}, d \varepsilon_{\beta}, d \boldsymbol{\beta}\right) d \mathcal{S}_{\mu}$

see [44] for additional details. The kinematically admissible displacement fluctuation space $\tilde{\mathscr{U}}_{\mu}^{S}$ in $\Omega_{\mu}$, and the corresponding virtual action space $\mathscr{V}_{\mu}^{S}$, are defined in expressions (35) and (37), respectively. The first constraint in (35) defines the field $d \tilde{\overline{\boldsymbol{u}}}_{\mu}$ satisfying (29) on $\Omega_{\mu}$. The second one defines the field $d \tilde{\boldsymbol{\beta}}_{\mu}$ satisfying (30) on $\mathcal{S}_{\mu}^{L}$.

The macro-scale admissible virtual actions are given in expression (36). In this case, note that the macroscale vector $\hat{\boldsymbol{\beta}}$ can be arbitrarily defined, while the macro-scale regular strain variation $\hat{\varepsilon}_{R}$ is assumed to be zero.

A specific Principle of Multi-scale Virtual Power (H2), valid for points $\boldsymbol{x}_{S}$ at the macro-scale, is postulated as follows:

$$
\begin{gathered}
\boldsymbol{T} \cdot \hat{\boldsymbol{\beta}}=\frac{1}{\left|\mathcal{S}_{\mu}^{L}\right|} \int_{\Omega_{\mu}} \boldsymbol{\sigma}_{\mu} \cdot \hat{\varepsilon}_{\mu}\left(\hat{\boldsymbol{\beta}}, \hat{\overline{\boldsymbol{u}}}_{\mu}, \hat{\boldsymbol{\beta}}_{\mu}^{1}, \ldots, \hat{\boldsymbol{\beta}}_{\mu}^{n_{c}}\right) d \Omega_{\mu}, \\
\forall \hat{\boldsymbol{\beta}} \quad \text { and } \quad \forall\left(\hat{\overline{\boldsymbol{u}}}_{\mu}, \hat{\boldsymbol{\beta}}_{\mu}^{1}, \ldots, \hat{\boldsymbol{\beta}}_{\mu}^{n_{c}}\right) \in \mathscr{V}_{\mu}^{S} .
\end{gathered}
$$

Two equations are naturally derived from the Variational Principle (31), see blocks (2.a) and (2.b) in Box 2:

(2.a) The first one is obtained by allowing arbitrary variations in $\hat{\boldsymbol{\beta}}$ and holding fixed $\left(\hat{\overline{\boldsymbol{u}}}_{\mu}, \hat{\boldsymbol{\beta}}_{\mu}^{1}, \ldots, \hat{\boldsymbol{\beta}}_{\mu}^{n_{c}}\right)$ to zero, resulting the expression (39) in Box 2. This expression provides the homogenization rule for the macro-scale traction $\boldsymbol{T}$ as the averaging of the micro-scale tractions $T_{\mathcal{S}_{\mu}^{L_{j}}}$ in all the opening cohesive cracks in $\Omega_{\mu}$. Note the presence of the parameter $\theta$ scaling the average traction in $\mathcal{S}_{\mu}^{L}$.

(2.b) Alternatively, by imposing arbitrary variation of $\left(\hat{\overline{\boldsymbol{u}}}_{\mu}\right.$, $\hat{\boldsymbol{\beta}}_{\mu}^{1}, \ldots, \hat{\boldsymbol{\beta}}_{\mu}^{n_{c}}$ ), and holding fixed $\hat{\boldsymbol{\beta}}$ to zero, the microscale equilibrium Eq. (40) is derived. This equation is similar, though with different kinematical constraints, to (28) in Box 1. 


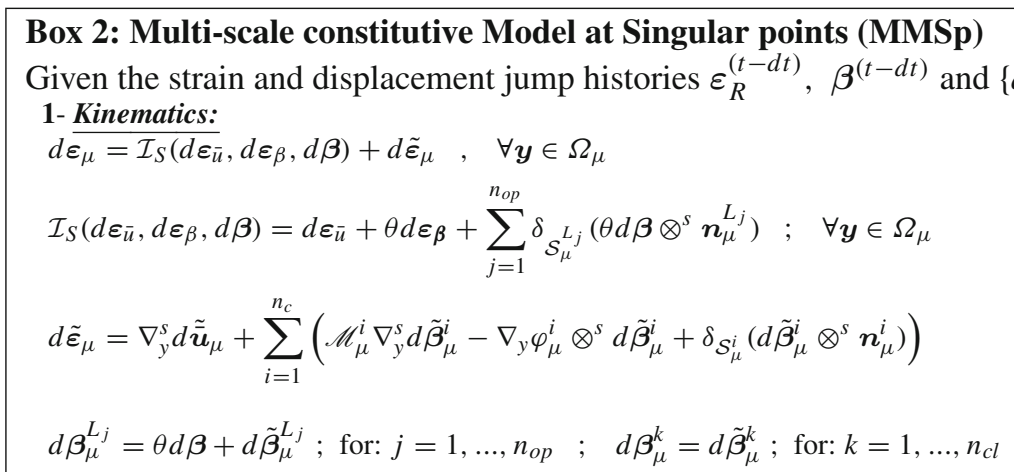

Kinematically admissible space of incremental displacement fluctuations, $d \tilde{\boldsymbol{u}}_{\mu}$ :

$$
\begin{aligned}
\tilde{\mathscr{U}}_{\mu}^{S}= & \left\{\left(d \tilde{\overline{\boldsymbol{u}}}_{\mu}, d \tilde{\boldsymbol{\beta}}_{\mu}^{1}, \ldots, d \tilde{\boldsymbol{\beta}}_{\mu}^{n_{c}}\right)\right. \text { suff. regular, such that: } \\
& \left.\int_{\Gamma_{\mu}} d \tilde{\overline{\boldsymbol{u}}}_{\mu} \otimes^{s} \boldsymbol{v}_{\mu} d \Gamma_{\mu}=\mathbf{0} \text { and } \sum_{j=1}^{n_{o p}} \int_{\mathcal{S}_{\mu}^{L_{j}}} d \tilde{\boldsymbol{\beta}}_{\mu}^{L_{j}} \otimes^{s} \boldsymbol{n}_{\mu}^{L_{j}} d \mathcal{S}_{\mu}=\mathbf{0}\right\}
\end{aligned}
$$

Virtual kinematically admissible actions:

Virtual actions at the macro-scale:

$$
\hat{\boldsymbol{\beta}} \text { arbitrary }, \quad \hat{\varepsilon}_{R} \equiv \mathbf{O}
$$

Virtual actions at the micro-scale:

$$
\begin{aligned}
& \left(\hat{\overline{\boldsymbol{u}}}_{\mu}, \hat{\boldsymbol{\beta}}_{\mu}^{1}, \ldots, \hat{\boldsymbol{\beta}}_{\mu}^{n_{c}}\right) \in \mathscr{V}_{\mu}^{S}, \quad \text { with: } \quad \mathscr{V}_{\mu}^{S}=\tilde{\mathscr{U}}_{\mu}^{S} \\
& \hat{\boldsymbol{\varepsilon}}_{\mu}=\mathcal{I}_{S}(\boldsymbol{O}, \boldsymbol{0}, \hat{\boldsymbol{\beta}})+\nabla_{y}^{s} \hat{\overline{\boldsymbol{u}}}_{\mu}+\sum_{i=1}^{n_{c}}\left(\mathscr{M}_{\mu}^{i} \nabla_{y}^{s} \hat{\boldsymbol{\beta}}_{\mu}^{i}-\nabla_{y}^{s} \varphi_{\mu}^{i} \otimes^{s} \hat{\boldsymbol{\beta}}_{\mu}^{i}+\delta_{\mathcal{S}_{\mu}^{i}}\left(\hat{\boldsymbol{\beta}}_{\mu}^{i} \otimes^{s} \boldsymbol{n}_{\mu}^{i}\right)\right)
\end{aligned}
$$

2-Consequences derived from the Principle of Multi-scale Virtual Power

2.a Traction Homogenization:

$$
\boldsymbol{T}=\frac{\theta}{\left|\mathcal{S}_{\mu}^{L}\right|}\left[\sum_{j=1}^{n_{o_{p}}} \int_{\mathcal{S}_{\mu}^{L_{j}}} \boldsymbol{T}_{\mathcal{S}_{\mu}^{L_{j}}} d \mathcal{S}_{\mu}\right]
$$

2.b Micro-scale Equilibrium problem:

$$
\begin{aligned}
& \text { Given the histories } \boldsymbol{\varepsilon}_{R}^{(t-d t)}, \boldsymbol{\beta}^{(t-d t)} \text { and }\left\{d \boldsymbol{\varepsilon}_{R}, d \boldsymbol{\beta}\right\} \text {, find }\left(d \tilde{\tilde{\boldsymbol{u}}}_{\mu}, d \tilde{\boldsymbol{\beta}}_{\mu}^{1}, \ldots, d \tilde{\boldsymbol{\beta}}_{\mu}^{n_{c}}\right) \in \tilde{\mathscr{U}}_{\mu}^{S} \text { such that: } \\
& \int_{\Omega_{\mu}} \boldsymbol{\sigma}_{\mu} \cdot \hat{\boldsymbol{\varepsilon}}_{\mu R} d \Omega_{\mu}+\sum_{i=1}^{n_{c}} \int_{\mathcal{S}_{\mu}^{i}} \boldsymbol{T}_{\mathcal{S}_{\mu}^{i}} \cdot \hat{\boldsymbol{\beta}}_{\mu}^{i} d \mathcal{S}_{\mu}=0 \quad, \quad \forall\left(\hat{\overline{\boldsymbol{u}}}_{\mu}, \hat{\boldsymbol{\beta}}_{\mu}^{1}, \ldots, \hat{\boldsymbol{\beta}}_{\mu}^{n_{c}}\right) \in \mathscr{V}_{\mu}^{S}
\end{aligned}
$$

Remark: note that both insertion operators $\mathcal{I}(\cdot)$, in Eqs. (21) and (33) define the information which is transferred from the macro to micro-scale, and the way in which the macro-scale kinematics is distributed in $\Omega_{\mu}$.

\section{Numerical implementation}

The numerical implementation of the multi-scale model, at the macro and micro-scale, are based on finite element methodologies. Although, in both scales it is assumed the existence of cohesive surfaces, and therefore, the modeling of strong discontinuities is required, different procedures are used in each case.

The macro-scale model is based on a finite element technique with embedded strong discontinuities (EFEM) and automatic crack path detection, recently introduced in the literature by Oliver et al. [46]. In that work, the novel EFEM technique has been utilized for mono-scale failure analysis. Its generalization to multi-scale analysis is here shown. In Sect. 3.1 a brief summary of the EFEM technique is presented. An important issue to be considered for implementing this element is the crack path tracking algorithm. Also, following to Oliver et al. [46], this algorithm is based on the "crack path field" technique, whose description is postponed until Sect. 3.3.

The present macro-scale model could be implemented using alternative finite element techniques dealing with strong discontinuities, such as XFEM, or tracking strategy for evaluating the crack path. It is noted that selection of the most convenient finite element technique, must be primarily 
decided according with the computational cost involved for evaluating the macro-scale integral terms, in Eq. (7). Specifically, we are referring to the number of quadrature points required by different techniques.

Alternatively, a standard finite element technique which uses stretched elements with very high aspect ratios, placed between all the edges of the conventional ones and mimicking cohesive surfaces, is adopted for modeling the micro-scale failure. This procedure is briefly summarized in Sect. 3.2. A discussion about the adequate choice of the RVE boundary conditions and their numerical implementation is also addressed in the same Section.

The finite element technique adopted for modeling the macro-scale is well adapted to handle strong discontinuities intersecting the mesh in arbitrary directions. However, considering that it requires important computational efforts, typically we refer to the evaluation of the crack path tracking algorithm, we prefer an approach computationally less demanding for solving the micro-scale, at the expense of giving up the ability to capture accurately the micro-scale crack path when it intersects the finite element mesh in arbitrary directions.

\subsection{Macro-scale finite element model: EFEM technique}

The finite element model is briefly described in Sect. 3.1.1, while the corresponding macro-scale discrete equilibrium equations are presented in Sect. 3.1.2.

\subsubsection{Finite element with strain enrichment: Constant Strain Mode (CSM) and Discontinuous Displacement Mode (DDM)}

Let us consider the mixed bilinear quadrilateral finite element technology with embedded strong discontinuities reported in Oliver et al. [46]. A finite element mesh covers the domain $\Omega^{h}$ of the body (in $\mathbb{R}^{2}$ ) and $\Omega^{e}$ denotes the domain of a specific finite element $e$.

During the structural loading process, the bilinear quadrilateral finite element $e$ can switch between three different formulations, denoted as states: 0,1 and 2 . The sets of elements with identical state define specific body subdomains, such as sketched in Fig. 8b. Elements in states 1 and 2 define the subdomains $\Omega_{C S M}^{h}$ and $\Omega_{D D M}^{h}$, respectively. A strong discontinuity surface $\mathcal{S}^{h}$ crosses the domain $\Omega_{D D M}^{h}$. The remaining subdomain $\Omega_{0}^{h}=\Omega^{h} \backslash\left(\Omega_{C S M}^{h} \cup \Omega_{D D M}^{h}\right)$ is defined by elements in state 0 .

Finite element formulations and the switching from one state to other are defined according with the following rules (see Fig. 8a):

- Initially, all elements in $\Omega^{h}$ are defined in state $0\left(\Omega_{0}^{h}=\right.$ $\left.\Omega^{h}\right)$. In this state, elements are modeled as standard quadrilaterals with continuous bilinear displacements. In the standard literature on the finite element method, this formulation is called irreducible. A quadrature rule using four Gauss points: $P G 1, \ldots, P G 4$, is implemented to evaluate the integrals arising from this irreducible formulation.

- Once the macro-scale bifurcation condition is detected at the pseudo-time $t=t_{N}$, in $\Omega^{e}$, the element originally in state 0 switches to state 1 and becomes part of the subdomain $\Omega_{C S M}^{h}$. The bifurcation condition in element $e$ is tested at the central point $x_{R}^{e}$ verifying the singularity of the homogenized acoustic tensor $Q^{\text {hom }}$. Then, the bifurcation criterion is satisfied, if:

$$
\operatorname{det}\left(\boldsymbol{Q}^{h o m}\right)=\operatorname{det}\left(\boldsymbol{n}_{b i f}^{e} \boldsymbol{C}^{h o m} \boldsymbol{n}_{b i f}^{e}\right)=0
$$

where $C^{\text {hom }}$ is the homogenized constitutive tensor given by Eq. (60) below, and $\boldsymbol{n}_{b i f}^{e}$ is one of the eigenvectors such that (41) is satisfied. The second non-trivial eigenvector is denoted $\gamma$ and represents the initial velocity jump direction across the possible singularity surface whose normal vector is $\boldsymbol{n}_{b i f}^{e}$.

Finite elements in state 1 are also quadrilaterals, but with mixed interpolation of displacements and strains: bilinear interpolation for the incremental displacements and constant interpolation for the incremental strains (Constant Strain Mode-CSM). So, a quadrature rule with only one integration point, the central one $x_{R}^{e}$ in Fig. $8 \mathrm{~b}$, is enough to evaluate consistently the integrals resulting from this mixed formulation.

Elements in state 1 are localized in the vicinity of the propagating crack tip (the fracture process zone). The objective pursued by introducing a CSM is to increase the flexibility of the finite elements, and thus, to get a better prediction of the crack path direction in that zone.

- After a subsequent, but small, increment of loading, at the pseudo-time $t_{S D}$ (with $t_{S D}>t_{N}$ ), finite elements in state 1 switch to state 2 and become part of subdomain $\Omega_{D D M}^{h}$. In state 2 , a strong discontinuity mode (Discontinuous Displacement Mode-DDM according to Oliver et al. [46]) is embedded into the quadrilateral finite element.

Previous to switching from states 1 to 2 , the position of the surface $\mathcal{S}^{e}(\boldsymbol{x})$ with normal vector $\boldsymbol{n}^{e}$ must be determined according to the technique given in Sect. 3.2 below.

Due to the incremental format of the multi-scale model in Sect. 2, the numerical integration can be consistently computed even when the quadrature rule is modified during the element switching from state 0 to 1 . 


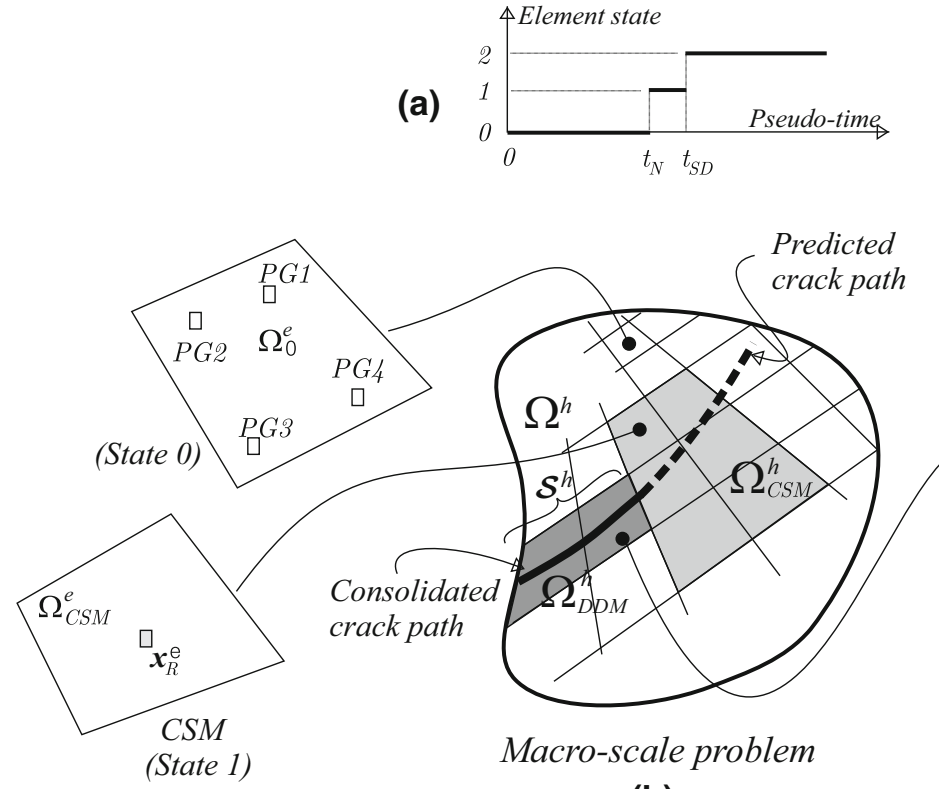

(b)

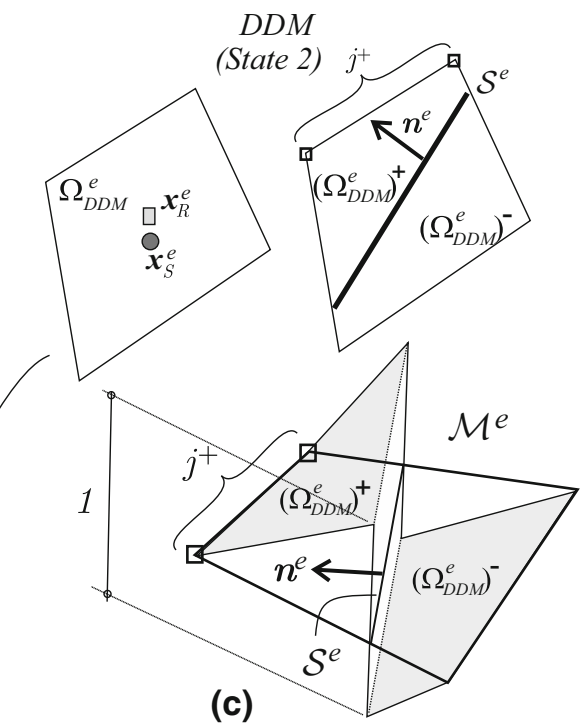

Fig. 8 Macro-scale finite element technique. a Relation between the pseudo-time versus finite element state for a particular macroscale finite element; b finite element states according with subdomain definitions, $\Omega_{C S M}^{h}$ is the domain with finite elements based on CSM (state 1) shown in light gray, $\Omega_{D D M}^{h}$ is the domain

The finite element formulations for states 0 and 1 have been implemented following standard procedures. Therefore, no further details are given, and the interested reader is referred to the above mentioned reference work. Conversely, finite elements in state 2 deserve an additional description which is presented in the following.

Implementation of the finite element with DDM (element in state 2). Let us consider the macro-scale finite element $e$, with subdomain: $\Omega_{D D M}^{e} \subset \Omega_{D D M}^{h}$, where an embedded strong discontinuity $\mathcal{S}^{e}$ with unit normal vector $\boldsymbol{n}^{e}$ is considered, see Fig. 8c. The discontinuous displacement increment field $d \boldsymbol{u}^{e}(\boldsymbol{x})$ in the element is given as follows:

$d \boldsymbol{u}^{e}(\boldsymbol{x})=\sum_{p=1}^{4} N_{p}^{e}(\boldsymbol{x}) d \overline{\boldsymbol{u}}^{p, e}+\mathscr{M}^{e}(\boldsymbol{x}) d \boldsymbol{\beta}^{e}$,

where $N_{p}^{e}$ is the standard shape function of the bilinear quadrilateral element related to the $p$-th node of element $e$. The vector of incremental parameters for the continuous displacement interpolation and associated with the same $p$-th node is denoted $d \overline{\boldsymbol{u}}^{p, e}$, while $d \boldsymbol{\beta}^{e}$ is the vector of element incremental parameters for the displacement jump interpolation. There is only one vector $d \boldsymbol{\beta}^{e}\left(\boldsymbol{\beta}^{e} \in \mathbb{R}^{2}\right)$ per element.

The unit jump function $\mathscr{M}^{e}(\boldsymbol{x})$ is depicted in Fig. $8 \mathrm{c}$ and is defined as follows:

with finite elements based on embedded strong discontinuitiesDDM (state 2) shown in deep gray, $\Omega^{h} \backslash\left(\Omega_{C S M}^{h} \cup \Omega_{D D M}^{h}\right)$ is the domain of elements based on an irreducible formulation (state $0)$ shown in white; c enrichment function $\mathscr{M}^{e}$ in elements with DDM

$\mathscr{M}^{e}(\boldsymbol{x})=\mathscr{H}^{e}(\boldsymbol{x})-\varphi^{e}(\boldsymbol{x})$,

where $\mathscr{H}^{e}$ is the Heaviside unit function shifted to $\mathcal{S}^{e}$, and:

$\varphi^{e}(\boldsymbol{x})=\sum_{j=1}^{j^{+}} N_{j}^{e}(\boldsymbol{x})$,

where the nodes $j=1, \ldots, j^{+}$belong to $\left(\Omega_{D D M}^{e}\right)^{+}$, as shown Fig. 8c.

A constant interpolation of regular strain increments per element is assumed. These strain increments are given in matrix notation as follows:

$$
\begin{gathered}
d \varepsilon_{R}^{e}(\boldsymbol{x})=\underbrace{\boldsymbol{B}^{e}\left(\boldsymbol{x}_{R}^{e}\right)\left[d \overline{\boldsymbol{u}}^{e}\right]}_{d \boldsymbol{\varepsilon}_{\bar{u}}^{e}}-\underbrace{\left[\nabla \varphi^{e}\right]\left(\boldsymbol{x}_{R}^{e}\right) d \boldsymbol{\beta}^{e}}_{\boldsymbol{d} \boldsymbol{\varepsilon}_{\beta}^{e}}, \\
\forall \boldsymbol{x} \in \Omega_{D D M}^{e} \backslash \mathcal{S}^{e}
\end{gathered}
$$

where the element vector $\left[\overline{\boldsymbol{u}}^{e}\right]$ collects all the displacements components for the finite element, $\boldsymbol{B}^{e}$ and $\left[\nabla \varphi^{e}\right]$ are the conventional displacement-strain matrix and the matrix gradient of the function $\varphi^{e}$. Both terms in (45) are evaluated at the central integration point $\boldsymbol{x}_{R}^{e}$. Using Voigt notation and a cartesian basis, the strain components are arranged as: $\varepsilon_{R}^{e}=\left[\varepsilon_{x_{1} x_{1}}, \varepsilon_{x_{2} x_{2}}, 2 \varepsilon_{x_{1} x_{2}}\right]^{T}$ (with subscripts $x_{1}$ and $x_{2}$ indicating the cartesian component in $\mathbb{R}^{2}$ ). The matrices in (45) are defined as follows: 


$$
\begin{gathered}
\boldsymbol{B}^{e}=\left[\begin{array}{ccccc}
\frac{\partial N_{1}^{e}}{\partial x_{1}} & 0 & \ldots & \frac{\partial N_{4}^{e}}{\partial x_{1}} & 0 \\
0 & \frac{\partial N_{1}^{e}}{\partial x_{2}} & \ldots & 0 & \frac{\partial N_{4}^{e}}{\partial x_{2}} \\
\frac{\partial N_{1}^{e}}{\partial x_{2}} & \frac{\partial N_{1}^{e}}{\partial x_{1}} & \ldots & \frac{\partial N_{4}^{e}}{\partial x_{2}} & \frac{\partial N_{4}^{e}}{\partial x_{1}}
\end{array}\right], \\
{\left[\nabla \varphi^{e}\right]=\left[\begin{array}{cc}
\frac{\partial \varphi^{e}}{\partial x_{1}} & 0 \\
0 & \frac{\partial \varphi^{e}}{\partial x_{2}} \\
\frac{\partial \varphi^{e}}{\partial x_{2}} & \frac{\partial \varphi^{e}}{\partial x_{1}}
\end{array}\right]}
\end{gathered}
$$

while the element vectors $\left[d \overline{\boldsymbol{u}}^{e}\right]$ and $d \boldsymbol{\beta}^{e}$ are ordered as:

$$
\begin{aligned}
{\left[d \overline{\boldsymbol{u}}^{e}\right] } & =\left[\left(d \bar{u}_{x_{1}}^{1}\right)\left(d \bar{u}_{x_{2}}^{1}\right) \ldots\left(d \bar{u}_{x_{1}}^{4}\right)\left(d \bar{u}_{x_{2}}^{4}\right)\right]^{T}, \\
d \boldsymbol{\beta}^{e} & =\left[d \beta_{x_{1}}^{e} d \beta_{x_{2}}^{e}\right]^{T} .
\end{aligned}
$$

The generalized strain increment in the singular point $\boldsymbol{x}_{S}^{e}$, positioned in the center of the element $\Omega_{D D M}^{e}$ and coincident with $\boldsymbol{x}_{R}^{e}$, as shown in Fig. 8c, is characterized through the kinematical descriptors:

$d \varepsilon_{S}^{e}\left(x_{S}^{e}\right)=\left(d \varepsilon_{\bar{u}}^{e}, d \varepsilon_{\beta}^{e}, d \beta^{e}\right)$,

where the first two descriptors are defined in (45).

In agreement with (42), the virtual actions in the domain of the $e$-th finite element, can be defined as:

$\hat{\boldsymbol{u}}^{e}=\sum_{p=1}^{4} N_{p}^{e} \hat{\overline{\boldsymbol{u}}}^{p, e}+\mathscr{M}^{e} \hat{\boldsymbol{\beta}}^{e}$,

where $\hat{\overline{\boldsymbol{u}}}^{p, e}$ and $\hat{\boldsymbol{\beta}}^{e}$ are the vector of parameters for the interpolation of continuous displacement and displacement jump virtual actions, respectively. Also, in agreement with (45), the virtual regular strains are:

$\hat{\varepsilon}_{R}^{e}=\boldsymbol{B}^{e}\left(\boldsymbol{x}_{R}^{e}\right)\left[\hat{\overline{\boldsymbol{u}}}^{e}\right]-\left[\nabla \varphi^{e}\right]\left(\boldsymbol{x}_{R}^{e}\right)\left[\hat{\boldsymbol{\beta}}^{e}\right]$.

Remark: only elements in state $2\left(\Omega_{D D M}^{e} \subset \Omega_{D D M}^{h}\right)$ are intersected by cracks. By construction, as shown in Fig. 8c, the support of enrichment functions $\varphi^{e}$ and $\mathscr{M}^{e}$ is $\Omega_{D D M}^{e}$. Then, elements having states 0 or $1\left(\Omega_{0}^{h} \cup \Omega_{C S M}^{h}\right)$ have the following particularities:

- they are outside the influence zone of any crack;

- in the insertion operator definition, equation (21), $d \varepsilon_{\beta}^{e}$ is identically zero;

- the insertion operator $\mathcal{I}_{R}\left(d \varepsilon_{\bar{u}}^{e}, \boldsymbol{O}\right)$ distributes uniformly the macro-strain $d \varepsilon_{\bar{u}}^{e}$ into the RVE domain;

- the MMRp procedure, which evaluates the constitutive relation $\sigma^{e}$ at all integration points, results identical to the conventional semi-concurrent homogenization techniques reported in the literature (see for example Miehe and Koch [47]), in the sense that the increment of macrostrain $d \varepsilon_{\bar{u}}^{e}$ is uniformly distributed into the RVE, and the overall stress $\sigma^{e}$ is the volumetric average in $\Omega_{\mu}$ of the micro-scale stresses $\sigma_{\mu}$ (the tortuosity factor has no influence in elements in states 0 and 1).

\subsubsection{Discrete equilibrium equations}

Introducing the strains and virtual actions (expressions (45) and (50) for finite elements with DDM) into the variational problem (7) and performing variations of $\hat{\overline{\boldsymbol{u}}}^{e}$, with $\hat{\boldsymbol{\beta}}^{e}$ fixed to zero, we obtain the discrete version of the equilibrium equation as follows:

$\underbrace{\Lambda_{e=1}^{\text {nelem }} \int_{\Omega^{e} \backslash \mathcal{S}^{e}}\left(\boldsymbol{B}^{e}\right)^{T} \boldsymbol{\sigma}^{e} d \Omega^{e}}_{\boldsymbol{F}^{\text {int }}}-\boldsymbol{F}^{e x t}=\boldsymbol{O}$,

where $\boldsymbol{F}^{\text {int }}$ and $\boldsymbol{F}^{\text {ext }}$ are the global vectors of internal and external forces, respectively, $\Lambda$ is the finite element assembling operator and $\sigma^{e}$ is the macro-scale stress at element $e$ arranged using Voigt notation. The vector $\boldsymbol{F}^{i n t}$ in Eq. (51) is evaluated with an adequate integration rule, according with the state of each finite element. Elements in state 0 require a standard integration rule with four Gauss points, while elements in states 1 and 2 require only one integration point (the integration point is placed at the central position of the element and is represented by $\boldsymbol{x}_{R}^{e}$ in Fig. 8).

Alternatively, the traction equilibrium equation across $\mathcal{S}^{e}$, with $\mathcal{S}^{e} \subset \Omega_{D D M}^{e} \subset \Omega_{D D M}^{h}$, is not consistently derived from (7). Instead, and following to Oliver et al. [48], a pointwise traction equilibrium equation (SKON formulation according with the denomination coined in the literature) is proposed as follows:

$\boldsymbol{T}^{e}\left(\boldsymbol{x}_{S}^{e}\right)=\boldsymbol{\sigma}^{e}\left(\boldsymbol{x}_{R}^{e}\right) \boldsymbol{n}^{e}, \quad \forall e \in \Omega_{D D M}^{h}$.

The traction vector $\boldsymbol{T}^{e}$ is evaluated at the integration point $\boldsymbol{x}_{S}^{e}$ attached to the cohesive surface, see Fig. 9. This vector is obtained by using the MMSp homogenization procedure described in Box 2, Eq. (39). On the other hand, the stress $\sigma^{e}$ is evaluated at the regular point $x_{R}^{e}$ using the MMRp homogenization procedure described in Box 1. There is only one Eq. (52) per each finite element $\Omega_{D D M}^{e}$ in state $2\left(\Omega_{D D M}^{e} \subset \Omega_{D D M}^{h}\right)$.

Note that according with the MMSp procedure sketched in Fig. 9, the strain localization domain $\mathcal{S}_{\mu}^{L}$ in the RVE associated with point $\boldsymbol{x}_{S}^{e}$, must be determined before nucleating the cohesive crack $\mathcal{S}^{e}$ at the macro-scale. In the numerical implementation, the evaluation of this domain is performed just before switching from state 1 to state 2 , at pseudo-time 


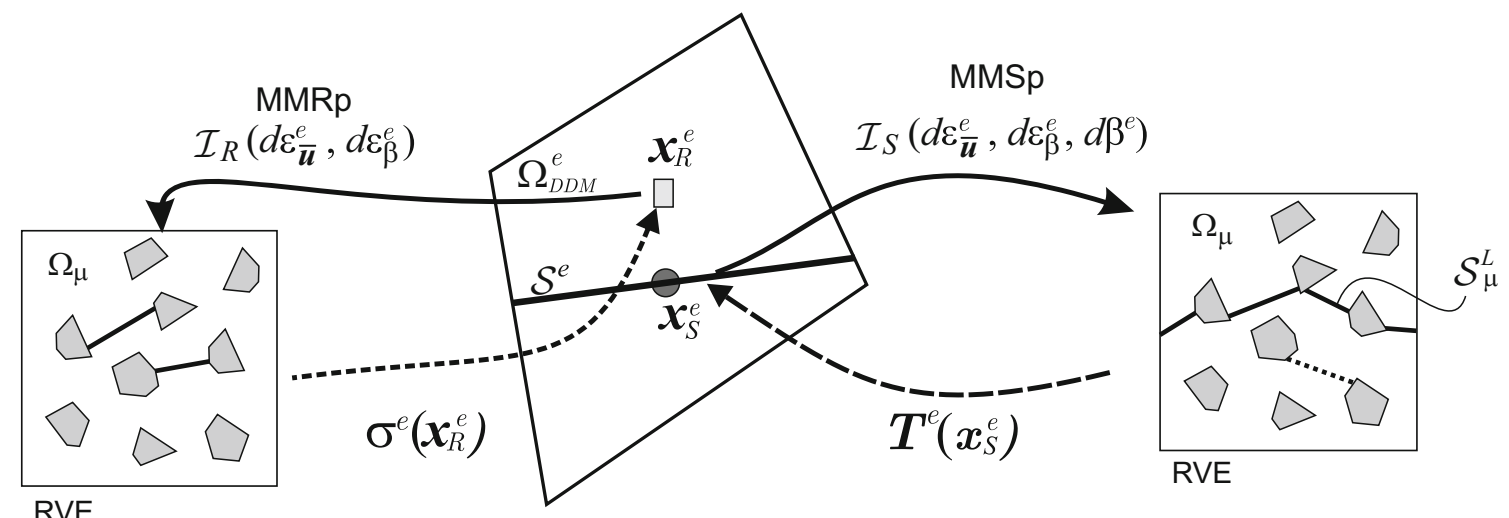

Fig. 9 Integration points of a finite element in state 2 with $\operatorname{DDM}\left(\Omega_{D D M}^{e} \subset \Omega_{D D M}^{h}\right)$. Regular $\left(\boldsymbol{x}_{R}^{e}\right)$ and singular $\left(\boldsymbol{x}_{S}^{e}\right)$ integration points

$t_{S D}$, with $t_{S D}>t_{N}$, see Fig. 8a. During the interval $\left[t_{N}, t_{S D}\right]$, the localization process at the RVE shows a fast development. So, the failure mechanism and the opening cohesive surfaces at the RVE are more clearly identified at $t_{S D}$ than at $t_{N}$, and the domain $\mathcal{S}_{\mu}^{L}$ can be thus more accurately determined.

Stiffness matrix of finite elements with DDM. The stiffness matrix of elements with DDM, in state 2, is derived from the equilibrium equations (51) and (52), and can be written as follows:

$\boldsymbol{K}^{e}=\left[\begin{array}{cc}\boldsymbol{K}_{\bar{u} \bar{u}}^{e} & \boldsymbol{K}_{\bar{u} \beta}^{e} \\ \boldsymbol{K}_{\beta \bar{u}}^{e} & \boldsymbol{K}_{\beta \beta}^{e}\end{array}\right]$,

where each sub-matrix is:

$\boldsymbol{K}_{\bar{u} \bar{u}}^{e}=\left[\boldsymbol{B}\left(\boldsymbol{x}_{R}^{e}\right)\right]^{T} \frac{\partial \boldsymbol{\sigma}^{e}\left(\boldsymbol{x}_{R}^{e}\right)}{\partial \overline{\boldsymbol{u}}^{e}}\left|\Omega^{e}\right|$,

$\boldsymbol{K}_{\bar{u} \beta}^{e}=-\left[\boldsymbol{B}\left(\boldsymbol{x}_{R}^{e}\right)\right]^{T} \frac{\partial \boldsymbol{\sigma}^{e}\left(\boldsymbol{x}_{R}^{e}\right)}{\partial \boldsymbol{\beta}^{e}}\left|\Omega^{e}\right|$,

$\boldsymbol{K}_{\beta \bar{u}}^{e}=\frac{\partial \boldsymbol{T}^{e}\left(\boldsymbol{x}_{S}^{e}\right)}{\partial \overline{\boldsymbol{u}}^{e}}-\boldsymbol{n}^{e} \frac{\partial \boldsymbol{\sigma}^{e}\left(\boldsymbol{x}_{R}^{e}\right)}{\partial \overline{\boldsymbol{u}}^{e}}\left|\Omega^{e}\right|$,

$\boldsymbol{K}_{\beta \beta}^{e}=\frac{\partial \boldsymbol{T}^{e}\left(\boldsymbol{x}_{S}^{e}\right)}{\partial \boldsymbol{\beta}^{e}}-\boldsymbol{n}^{e} \frac{\partial \boldsymbol{\sigma}^{e}\left(\boldsymbol{x}_{R}^{e}\right)}{\partial \boldsymbol{\beta}^{e}}\left|\Omega^{e}\right|$,

where the symbol $\left|\Omega^{e}\right|$ denotes the area of the finite element. The stress derivatives in these expressions are found by considering the stress homogenization Eq. (27) and the macro-scale regular strain (see Eq. 45):

$\frac{\partial \boldsymbol{\sigma}^{e}\left(\boldsymbol{x}_{R}^{e}\right)}{\partial \overline{\boldsymbol{u}}^{e}}=\boldsymbol{C}^{\text {hom }} \boldsymbol{B}^{e}$,

$\frac{\partial \boldsymbol{\sigma}^{e}\left(\boldsymbol{x}_{R}^{e}\right)}{\partial \boldsymbol{\beta}^{e}}=-\theta \boldsymbol{C}^{h o m}\left[\nabla \varphi^{e}\right]$,

where the homogenized tangent tensor $C^{\text {hom }}$ is given by:
$C^{\text {hom }}=\frac{1}{\left|\Omega_{\mu}\right|} \int_{\Omega_{\mu}}\left(\boldsymbol{C}_{\mu}+\boldsymbol{C}_{\mu} \mathcal{A}(\boldsymbol{y})\right) d \Omega_{\mu}$,

$\mathcal{A}(\boldsymbol{y})=\frac{\partial \tilde{\varepsilon}_{\mu}}{\partial \varepsilon_{R}^{e}}, \quad \mathcal{A} \in \mathbb{R}^{3 \times 3}$.

The operator $\mathcal{A}$ is derived from the implicit functional dependence $\tilde{\varepsilon}_{\mu}\left(\varepsilon_{R}^{e}\right)$ established through the micro-scale equilibrium problems (28), see Box 1. Then, the linear operator $\mathcal{A}(\boldsymbol{y})$ can be determined by solving three linear micro-scale equilibrium problems (in $\mathbb{R}^{2}$ ). See additional details in [22] and [43].

Finally, the traction homogenization expression (39) in Box 2 related to the MMSp procedure can be derived, resulting in the following expressions:

$$
\begin{aligned}
& \frac{\partial \boldsymbol{T}^{e}}{\partial \overline{\boldsymbol{u}}^{e}}=\frac{\theta}{\left|\mathcal{S}_{\mu}^{L}\right|} \sum_{j=1}^{n_{o p}} \int_{\mathcal{S}_{\mu}^{L_{j}}} \boldsymbol{n}_{\mu}^{L_{j}} \boldsymbol{C}_{\mu} \mathcal{D}(\boldsymbol{y}) d \mathcal{S}_{\mu}, \\
& \frac{\partial \boldsymbol{T}^{e}}{\partial \boldsymbol{\beta}^{e}}=\frac{\theta}{\left|\mathcal{S}_{\mu}^{L}\right|} \sum_{j=1}^{n_{o p}} \int_{\mathcal{S}_{\mu}^{L_{j}}}-\boldsymbol{n}_{\mu}^{L_{j}}\left(\boldsymbol{C}_{\mu}+\boldsymbol{C}_{\mu} \mathcal{C}(\boldsymbol{y})\right) d \mathcal{S}_{\mu},
\end{aligned}
$$

where we have defined:

$\mathcal{D}(\boldsymbol{y})=\frac{\partial \tilde{\boldsymbol{\beta}}_{\mu}}{\partial \overline{\boldsymbol{u}}^{e}}, \quad \mathcal{C}(\boldsymbol{y})=\frac{\partial \tilde{\boldsymbol{\beta}}_{\mu}}{\partial \boldsymbol{\beta}^{e}}$,

which can be computed by considering the implicit functional dependence $d \tilde{\boldsymbol{\beta}}_{\mu}\left(d \varepsilon_{R}^{e}, d \boldsymbol{\beta}^{e}\right)$ given by equation (40). Both operators can be found by solving two micro-scale equilibrium problems.

\subsection{Micro-scale finite element model}

The finite element model at the micro-scale is implemented using a conventional technique without appealing to the explicit inclusion of strong discontinuities. Let us consider the finite element domain of the RVE, denoted $\Omega_{\mu}^{h}$, with 


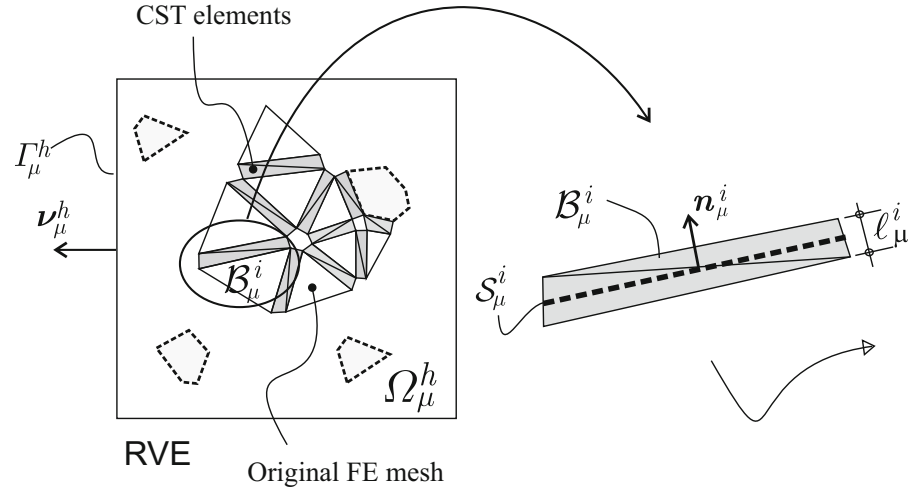

(b)

(a)

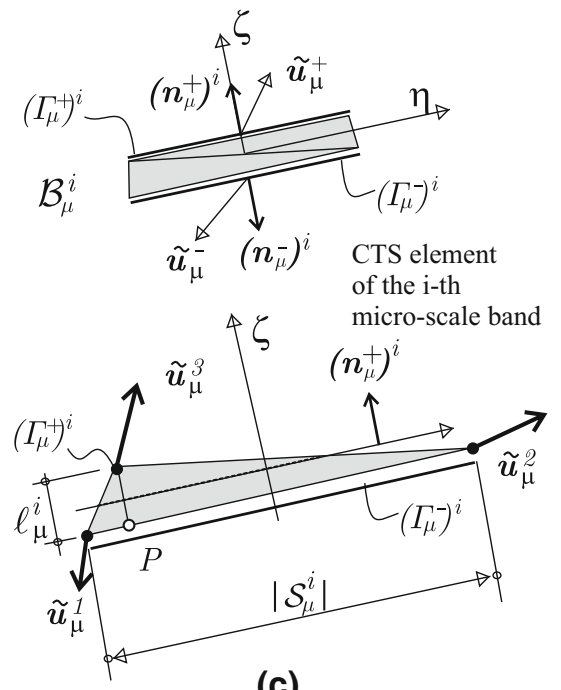

(c)

Fig. 10 RVE finite element model. a CST finite elements arranged like thin bands are inserted between the edges of original finite elements (the band-like domains are depicted in grey); b band $\mathcal{B}_{\mu}^{i}$ and its associated

boundary $\Gamma_{\mu}^{h}$ and unit normal vector $\nu_{\mu}^{h}$, as shown in Fig. 10a. The micro-scale displacement fluctuation increment field, $d \tilde{\boldsymbol{u}}_{\mu}$, is interpolated in $\Omega_{\mu}^{h}$ as follows:

$d \tilde{\boldsymbol{u}}_{\mu}(\boldsymbol{y})=\sum_{k=1}^{n_{\text {nod }}} N_{\mu_{k}}(\boldsymbol{y}) d \tilde{\boldsymbol{q}}_{\mu}^{k}$,

where $n_{n o d}$ is the number of nodes of the micro-scale finite element mesh, $N_{\mu_{k}}$ the shape function of the $k$-th node and $\tilde{\boldsymbol{q}}_{\mu}^{k}$ the vector of interpolation parameters, related to the same $k$-th node, for the displacement fluctuations.

The micro-scale strain increment in the finite element $e_{\mu}$ is:

$d \varepsilon_{\mu}^{e_{\mu}}(\boldsymbol{y})=\mathcal{I}(d \varepsilon)+\underbrace{\boldsymbol{B}_{\mu}^{e_{\mu}}(\boldsymbol{y})\left[d \tilde{\boldsymbol{q}}_{\mu}^{e_{\mu}}\right]}_{d \tilde{\boldsymbol{\varepsilon}}_{\mu}^{e_{\mu}}}$,

where the insertion operator $\mathcal{I}(\cdot)$ is defined in (21), or (33), depending on the macro-scale point to which the RVE is associated with, and $\boldsymbol{B}_{\mu}^{e_{\mu}}$ is the conventional strain-displacement matrix for the micro-scale finite element $e_{\mu}$. The last term in (65) is the micro-scale strain fluctuation increment $d \tilde{\varepsilon}_{\mu}^{e_{\mu}}(\boldsymbol{y})$. The vector $\left[d \tilde{\boldsymbol{q}}_{\mu}^{e_{\mu}}\right]$ collects all the interpolation parameters of the finite element for the displacements fluctuation increments.

\subsubsection{Use of two-dimensional thin band-like domains}

Two-dimensional Constant Strain Triangles (CST) elements, displaying high aspect ratios and distributed forming thin band-like domains, are inserted between all the element geometrical entities (thickness $\ell_{\mu}^{i}$, normal vector $\boldsymbol{n}_{\mu}^{i}$ and mean surface $\left.\mathcal{S}_{\mu}^{i}\right)$; c local coordinate system $(\eta, \zeta)$ of the $i$-th band and displacement fluctuation vectors in the CST element nodes

edges of the original finite element mesh, such as shown in Fig. 10.

Each so-formed thin band $\mathcal{B}_{\mu}^{i}$ (superscript $i$ identifies the band number) has associated a thickness $\ell_{\mu}^{i}$, a normal vector $\boldsymbol{n}_{\mu}^{i}$ and a mean surface $\mathcal{S}_{\mu}^{i}$, as shown in Fig. 10b. A band area $\left|\mathcal{S}_{\mu}^{i}\right|$ (or length per unit of thickness) can be defined, such that $\left|\mathcal{B}_{\mu}^{i}\right|=\left|\mathcal{S}_{\mu}^{i}\right| \ell_{\mu}^{i}$, where $\left|\mathcal{B}_{\mu}^{i}\right|$ is the volume of the corresponding band. The geometrical distribution of CST elements forming the bands are shown in gray in Fig. 10, and their aspect ratio increases with $\ell_{\mu}^{i} \rightarrow 0$.

The displacement fluctuation and strain fields into the bands are described by equations (64) and (65), respectively.

The objective of introducing these micro-scale bands into the mesh is to capture the failure mechanisms induced by strain localization effects. This procedure can be considered as an alternative technique to more standard cohesive interface finite elements. A distinguishing feature in the present approach is that we can assign continuum constitutive models to the material constituting the thin micro-scale bands.

Strain localization is forced to happens exclusively into the bands $\mathcal{B}_{\mu}^{i}$ by defining there a regularized strain softening material response, while an elastic response is adopted for all the constituents of the composite. Strain localization into the bands initiates after the micro-scale bifurcation condition is reached.

An additional concept associated with the band $\mathcal{B}_{\mu}^{i}$ is introduced in the present finite element model. Let us define a local orthogonal coordinate system $(\eta, \zeta)$ aligned with the band, $\eta$ is placed along the line resulting from the intersec- 
tion of $\mathcal{S}_{\mu}^{i}$ with the plane of analysis, as shown in Fig. 10c. Let us also consider the displacement jump fluctuation increment across the band, $d \tilde{\boldsymbol{\beta}}_{\mu}^{i}$, defined along the coordinate $\eta$ as follows:

$$
\begin{aligned}
d \tilde{\boldsymbol{\beta}}_{\mu}^{i}(\eta) & =d \tilde{\boldsymbol{u}}_{\mu}\left(\zeta^{+}, \eta\right)-d \tilde{\boldsymbol{u}}_{\mu}\left(\zeta^{-}, \eta\right) \\
& =d \tilde{\boldsymbol{u}}_{\mu}^{+}(\eta)-d \tilde{\boldsymbol{u}}_{\mu}^{-}(\eta)
\end{aligned}
$$

where the coordinate $\zeta^{+}$indicates the position on the boundary $\left(\Gamma_{\mu}^{+}\right)^{i}$ and $\zeta^{-}$the position on the boundary $\left(\Gamma_{\mu}^{-}\right)^{i}$. The surfaces $\left(\Gamma_{\mu}^{+}\right)^{i}$ and $\left(\Gamma_{\mu}^{-}\right)^{i}$ limit the band and are depicted in the same Figure. According with the notation in Fig. 10c, it is noted that the micro-scale strain fluctuation increment in the CST element belonging to $\mathcal{B}_{\mu}^{i}$, is given by (see additional details in the work of Manzoli et al. [49]):

$d \tilde{\varepsilon}_{\mu}^{e_{\mu}}=\frac{d \tilde{\boldsymbol{\beta}}_{\mu}^{i *} \otimes^{s} \boldsymbol{n}_{\mu}^{+i}}{\ell_{\mu}^{i}}+\mathcal{O}\left(\frac{\left\|d \tilde{\boldsymbol{q}}_{\mu}^{2}-d \tilde{\boldsymbol{q}}_{\mu}^{1}\right\|}{\left|\mathcal{S}_{\mu}^{i}\right|}\right)$,

where $d \tilde{\boldsymbol{\beta}}_{\mu}^{i *}=d \tilde{\boldsymbol{q}}_{\mu}^{3}-d \tilde{\boldsymbol{u}}_{\mu}^{P}$, being $d \tilde{\boldsymbol{u}}_{\mu}^{P}$ the displacement fluctuation increment interpolated at point $P$. The point $P$ is the orthogonal projection of node 3 onto $\left(\Gamma_{\mu}^{-}\right)^{i}$. The second term in (67) can be neglected when $\ell_{\mu}^{i} \ll\left|\mathcal{S}_{\mu}^{i}\right|$ and the band initiates the strain localization process. In this case, the micro-scale strain fluctuation increment $d \tilde{\varepsilon}_{\mu}^{e_{\mu}}$ interpolated by (65) into the CST element, naturally approaches to:

$d \tilde{\boldsymbol{\beta}}_{\mu}^{i *} \approx \frac{d \tilde{\boldsymbol{\beta}}_{\mu}^{i *} \otimes^{s} \boldsymbol{n}_{\mu}^{+i}}{\ell_{\mu}^{i}}$

Constitutive relation for the micro-scale bands. The continuum damage model presented in Box 3 describes the material response of the bands $\mathcal{B}_{\mu}^{i}$. This constitutive relation has been taken from Oliver [18].

According with Eq. (69), the internal variables of the model, $q_{\mu}$ and $r_{\mu}$, as well as their evolution laws, Eqs. (72)(73), determine the isotropic damage, $d_{\mu}$, which defines the degradation of the original elastic response ruled by the elastic constitutive tensor $C_{\mu}^{e}$. Initial values of $q_{\mu}$ and $r_{\mu}$ are $q_{\mu 0}$ and $r_{\mu 0}$, respectively. The variable $r_{\mu}$ can be seen as the maximum value of a strain norm reached during the loading history.

The relation between $\dot{r}_{\mu}$ and $\dot{q}_{\mu}$ is given by the softening modulus $H_{\mu}<0$, as shown in Eq. (72). This parameter is characterized from the fracture energy, $G_{\mu}^{f}$, of the microscale material constituent. The remarkable aspect of the damage model in Box 3 is the regularization of the softening modulus $H_{\mu}$, with the parameter $\ell_{\mu}$, as described by Eq. (73).

The positive counterpart of the effective micro-scale stress, $\left(\boldsymbol{\sigma}_{\mu}^{\text {eff }}\right)^{+}$, is introduced in the damage criterion (70) with the objective of modeling damage evolution only under tensile stress condition.

By adopting this continuum damage model for the microscale bands $\mathcal{B}_{\mu}^{i}$, with $\ell_{\mu}^{i}$ small compared to the micro-cell size, and considering that:

(a) the softening modulus of the damage constitutive relation is regularized with the band thickness $\ell_{\mu}^{i}$, so that the total dissipated energy does not depend of this length (this is the reason for introducing the length $\ell_{\mu}^{i}$ into the definition of $H_{\mu}$, as shown in equation (73), Box 3);

(b) its elastic response is identical to that of the neighbor bulk material, so that the overall RVE elastic response is independent of the size $\ell_{\mu}^{i}$;

then, it can be concluded that the complete response of the $\mathrm{RVE}$ is independent of the value adopted for $\ell_{\mu}^{i}$.

Identification of the strain localization domain at $t=t_{S D}$. Once the singularity of the corresponding acoustic tensor has been verified in the CST element $\mathcal{B}_{\mu}^{i}$, at time $t_{\mu N}^{i}$ (with $t_{\mu N}^{i}<t_{N}$ ), the band is identified as a nucleated micro-scale band. Considering that there are $n_{c}$ nucleated bands, the complete domain of nucleated bands is denoted $\mathcal{B}_{\mu}$, being $\mathcal{B}_{\mu}=\cup_{i=1}^{n_{c}} \mathcal{B}_{\mu}^{i}$.

An additional ingredient introduced in the RVE finite element model refers to the opening strain localization domain, denoted $\mathcal{B}_{\mu}^{L}$, see Fig. 11. This domain is defined after the macro-scale bifurcation condition has been detected at $t=$ $t_{N}$. Typically, $\mathcal{B}_{\mu}^{L}$ is evaluated at $t=t_{S D}$ (with $t_{S D}>t_{N}$ as shown in Fig. 8a). As mentioned in Sect. 3.1.1, the solution of Eq. (41) provides two macro-scale eigenvectors, $\boldsymbol{n}_{b i f}^{e}$ and $\gamma$. It is assumed that a nucleated band $\mathcal{B}_{\mu}^{i}$ is opening at $t=t_{S D}$ if: $d \tilde{\varepsilon}_{\mu} \cdot\left(\boldsymbol{n}_{b i f}^{e} \otimes \gamma\right)>0$, where $d \tilde{\varepsilon}_{\mu}$ is the micro-scale strain fluctuation increment evaluated in the CST element of the band. In this case, the band is identified with the notation $\mathcal{B}_{\mu}^{L_{j}}$. Contrarily, a nucleated micro-scale band is closing at $t=t_{S D}$ if that condition is not satisfied.

Then, $\mathcal{B}_{\mu}^{L}$ is defined by $\mathcal{B}_{\mu}^{L}=\cup_{j=1}^{n_{\text {op }}} \mathcal{B}_{\mu}^{L_{j}}$, where $n_{\text {op }}$ is the number of opening micro-cracks at $t=t_{S D}$. The number of nucleated micro-scale bands which are closing at $t=t_{S D}$ is $n_{c l}=n_{c}-n_{o p}$.

Note that every element in $\mathcal{B}_{\mu}$ is identified with the superscript " $i$ " $\left(\mathcal{B}_{\mu}^{i}\right)$, while, elements in $\mathcal{B}_{\mu}^{L}$ are identified with the superscript " $L_{j}$ ", where the index " $j$ " denotes one specific band of the set. According with this notation, all the geometrical entities associated with the band $\mathcal{B}_{\mu}^{L_{j}}$ are denoted with superscript “ $L_{j}$ ”, e.g.: $\boldsymbol{n}_{\mu}^{L_{j}}, \mathcal{S}_{\mu}^{L_{j}}, \ell_{\mu}^{L_{j}}$.

Identification of the macro-scale normal vector $\boldsymbol{n}^{e}$ at $t=$ $t_{S D}$. The macro-scale normal vector $\boldsymbol{n}^{e}$, used in the implementation of the EFEM technique, is also evaluated at $t=$ $t_{S D}$ as follows: 


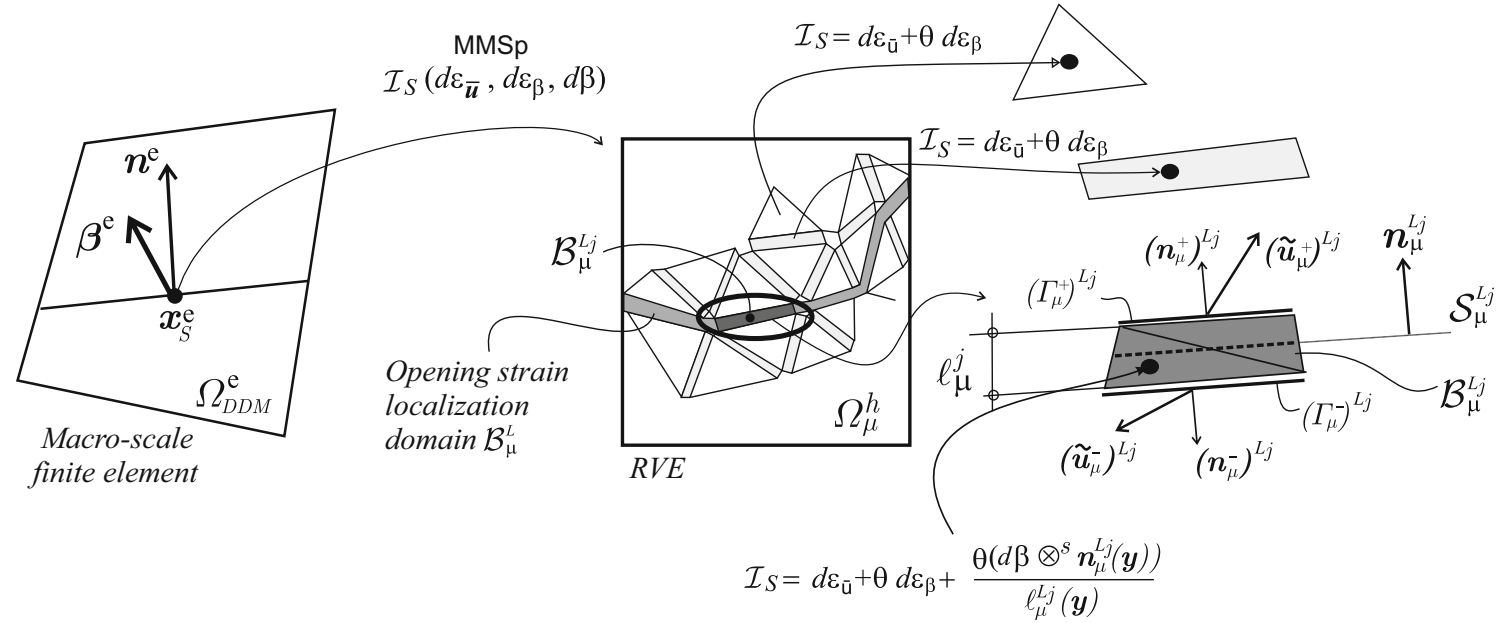

Fig. 11 Operator $\mathcal{I}_{S}$ used in the MMSp homogenization procedure. Macro-scale strain insertion into the micro-scale finite element mesh, $\Omega_{\mu}^{h}$

\section{Box 3: Regularized tensile damage model at the micro-scale.}

Elastic stress-strain relation

$$
\boldsymbol{\sigma}_{\mu}=\frac{q_{\mu}}{r_{\mu}} \boldsymbol{C}_{\mu}^{e} \varepsilon_{\mu}=\left[1-d_{\mu}\left(r_{\mu}\right)\right] \overbrace{\boldsymbol{C}_{\mu}^{e} \varepsilon_{\mu}}^{\boldsymbol{\sigma}_{\mu}^{e f f}} \quad, \quad d_{\mu}\left(r_{\mu}\right)=1-\frac{q_{\mu}\left(r_{\mu}\right)}{r_{\mu}} .
$$

Damage criterion

$$
\mathscr{G}\left(\varepsilon_{\mu}, r_{\mu}\right)=\sqrt{\left(\boldsymbol{\sigma}_{\mu}^{\text {eff }}\right)^{+} \cdot \varepsilon_{\mu}}-r_{\mu} \leq 0 \quad, \quad\left(\boldsymbol{\sigma}_{\mu}^{\text {eff }}\right)^{+}=\sum_{i=1}^{3}\left\langle\left(\sigma_{\mu}^{\text {eff }}\right)_{i}\right\rangle \boldsymbol{e}_{i} \otimes \boldsymbol{e}_{i} .
$$

Loading/Unloading complementary conditions

$$
\dot{r}_{\mu} \geq 0, \quad \mathscr{G} \leq 0, \quad \dot{r}_{\mu} \mathscr{G}=0 \quad,\left.\quad r_{\mu}\right|_{t=0}=r_{\mu 0}=\frac{\sigma_{\mu}^{u}}{\sqrt{E_{\mu}}} .
$$

Internal variable evolution laws with softening

$$
\begin{aligned}
& \dot{q}_{\mu}=H_{\mu}\left(r_{\mu}\right) \dot{r}_{\mu}, \quad q_{\mu} \geq 0,\left.\quad q_{\mu}\right|_{t=0}=q_{\mu 0}=r_{\mu 0}, \\
& H_{\mu}\left(r_{\mu}\right)=-\frac{\ell_{\mu} r_{\mu 0}^{2}}{G_{\mu}^{f}} \exp \left[-\frac{\ell_{\mu} r_{\mu 0}}{G_{\mu}^{f}}\left(r_{\mu}-r_{\mu 0}\right)\right] .
\end{aligned}
$$

\section{Tangent constitutive tensor}

$$
\begin{aligned}
& \text { if } \dot{r}_{\mu}=0, \quad \boldsymbol{C}_{\mu}=\left(1-d_{\mu}\right) \boldsymbol{C}_{\mu}^{e} \\
& \text { if } \dot{r}_{\mu}>0, \quad \boldsymbol{C}_{\mu}=\left(1-d_{\mu}\right) \boldsymbol{C}_{\mu}^{e}-\left[\frac{q_{\mu}-H_{\mu} r_{\mu}}{r_{\mu}^{3}}\right] \boldsymbol{\sigma}_{\mu}^{\text {eff }} \otimes\left(\boldsymbol{\sigma}_{\mu}^{\text {eff }}\right)^{+} .
\end{aligned}
$$

\section{Material parameters}

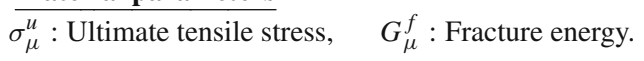

$E_{\mu}, v_{\mu}$ : Young's modulus and Poisson ratio, defining the elastic constitutive tensor $C_{\mu}^{e}$

\section{Definitions of variables}

$r_{\mu}:$ strain-like internal variable,

$q_{\mu}:$ stress-like internal variable,

$d_{\mu}:$ scalar damage variable,

$H_{\mu}$ : softening modulus (exponential degradation).

$\boldsymbol{\sigma}_{\mu}^{e f f}$ : effective stress,

$\left(\sigma_{\mu}^{e f f}\right)_{i}: i$-th principal stress of $\boldsymbol{\sigma}_{\mu}^{e f f}$,

$\boldsymbol{e}_{i}: i$-th eingenvector of $\boldsymbol{\sigma}_{\mu}^{e f f}$,

$\left(\sigma_{\mu}^{e f f}\right)^{+}$: positive counterpart of effective stress,

$\langle\cdot\rangle$ : Macaulay brackets, $\quad \ell_{\mu}$ : strain localization band thickness, 


$$
\boldsymbol{n}^{e}=\frac{\sum_{j=1}^{n_{o p}} \int_{\mathcal{S}_{\mu}^{L_{j}}} \boldsymbol{n}_{\mu}^{L_{j}} d \mathcal{S}_{\mu}}{\left\|\sum_{j=1}^{n_{o p}} \int_{\mathcal{S}_{\mu}^{L_{j}}} \boldsymbol{n}_{\mu}^{L_{j}} d \mathcal{S}_{\mu}\right\|}
$$

Considering the tortuosity parameter defined in Eq. (17), it is noted that $\boldsymbol{n}^{e}$ is the average vector obtained by integrating $\boldsymbol{n}_{\mu}^{L_{j}}$ along $\mathcal{S}_{\mu}^{L}$ and scaled by $\theta$, such that $\left\|\boldsymbol{n}^{e}\right\|=1$. The evaluation of $\boldsymbol{n}^{e}$ with Eq.(76) requires that the orientations of $\boldsymbol{n}_{\mu}^{L_{j}}$ are defined following the rule:

$\boldsymbol{n}_{\mu}^{L_{j}} \cdot \boldsymbol{n}_{b i f}^{e}>0, \quad \forall j=1, \ldots, n_{o p}$,

where $\boldsymbol{n}_{b i f}^{e}$ is one of the eigenvector of the macro-scale bifurcation problem in Eq. (41).

According with our numerical experience, see the results presented in next Sect. 4.2, the difference observed between the so-determined vector $\boldsymbol{n}^{e}$ and $\boldsymbol{n}_{b i f}^{e}$ does not exceed a few degrees.

\subsubsection{Implementation of the insertion operators}

In the forthcoming developments, the superscript " $e$ " indicating macro-scale finite element is removed from the inserted macro-scale kinematical objects.

In expression (65), the operator $\mathcal{I}(\cdot)$ inserts the macroscale strain according with the procedure defined in Sect. 2.3. When the MMRp homogenization procedure is used, Eq. (21) indicates that the operator $\mathcal{I}_{R}(\cdot)$ inserts uniformly the macro-scale strain increment, $d \varepsilon_{\bar{u}}+\theta d \varepsilon_{\beta}$, in all elements of $\Omega_{\mu}^{h}$. This uniform distribution includes all the CST band elements (even those corresponding to not nucleated bands).

Alternatively, when the MMSp procedure is used, the operator $\mathcal{I}_{S}(\cdot)$ also inserts uniformly the macro-scale strain increment, $d \varepsilon_{\bar{u}}+\theta d \varepsilon_{\beta}$, in all elements of $\Omega_{\mu}^{h}$ (including all the CST elements of the $n_{c}$ bands), but additionally it inserts the term $\theta d \boldsymbol{\beta}$ only in the strain localization domain $\mathcal{B}_{\mu}^{L}$. Therefore, the maro-scale strain increment inserted into a CST element in the band $\mathcal{B}_{\mu}^{L_{j}}$ is (see Fig. 11):

$$
\mathcal{I}_{S}\left(d \varepsilon_{\bar{u}}, d \varepsilon_{\boldsymbol{\beta}}, d \boldsymbol{\beta}\right)=d \varepsilon_{\bar{u}}+\theta d \varepsilon_{\boldsymbol{\beta}}+\left[\frac{\theta d \boldsymbol{\beta} \otimes^{s} \boldsymbol{n}_{\mu}^{L_{j}}}{\ell_{\mu}^{L_{j}}}\right] .
$$

\subsubsection{Displacement fluctuations and virtual action fields}

According with Eq. (23) and the displacement field interpolation (64), we define the discrete space of displacement fluctuation increments for the MMRp homogenization procedure, $\tilde{\mathscr{U}}_{\mu}^{R h}$ in $\Omega_{\mu}^{h}$ as follows:

$$
\begin{aligned}
\tilde{\mathscr{U}}_{\mu}^{R h}= & \left\{d \tilde{\boldsymbol{u}}_{\mu}(\boldsymbol{y})=\sum_{k=1}^{n_{\text {nod }}} N_{\mu k}(\boldsymbol{y}) d \tilde{\boldsymbol{q}}_{\mu}^{k},\right. \\
& \text { such that } \left.\int_{\Gamma_{\mu}^{h}} d \tilde{\boldsymbol{u}}_{\mu} \otimes^{s} \boldsymbol{\nu}_{\mu}^{h} d \Gamma_{\mu}=\boldsymbol{0}\right\} .
\end{aligned}
$$

The kinematical constraint in (79) ensures a zero average value, in $\Omega_{\mu}^{h}$, of the micro-scale strain fluctuation increment field $d \tilde{\varepsilon}_{\mu}$, which is defined in every finite element $e_{\mu}$ as:

$d \tilde{\varepsilon}_{\mu}^{e_{\mu}}=\boldsymbol{B}_{\mu}^{e_{\mu}}(\boldsymbol{y})\left[d \tilde{\boldsymbol{q}}_{\mu}^{e_{\mu}}\right]$.

The discrete space of virtual displacement actions $\mathscr{V}_{\mu}^{R h}$ is:

$$
\begin{aligned}
\mathscr{V}_{\mu}^{R h}= & \left\{\hat{\boldsymbol{u}}_{\mu}(\boldsymbol{y})=\sum_{k=1}^{n_{\text {nod }}} N_{\mu k}(\boldsymbol{y}) \hat{\boldsymbol{q}}_{\mu}^{k},\right. \\
& \text { such that } \left.\int_{\Gamma_{\mu}^{h}} \hat{\boldsymbol{u}}_{\mu} \otimes^{s} \boldsymbol{\nu}_{\mu}^{h} d \Gamma_{\mu}=\mathbf{0}\right\} .
\end{aligned}
$$

On the other hand, by considering the MMSp homogenization procedure and according with (35) and (64), the discrete space of incremental displacement fluctuations, $\tilde{\mathscr{U}}_{\mu}^{S h}$ in $\Omega_{\mu}^{h}$, is defined as follows:

$$
\begin{aligned}
\tilde{\mathscr{U}}_{\mu}^{S h}=\mathscr{V}_{\mu}^{S h}= & \left\{d \tilde{\boldsymbol{u}}_{\mu}(\boldsymbol{y})=\sum_{k=1}^{n_{n o d}} N_{\mu k}(\boldsymbol{y}) d \tilde{\boldsymbol{q}}_{\mu}^{k},\right. \\
& \text { such that } \int_{\Gamma_{\mu}^{h}} d \tilde{\boldsymbol{u}}_{\mu} \otimes^{s} \boldsymbol{\nu}_{\mu}^{h} d \Gamma_{\mu}=\boldsymbol{0} \\
& \text { and } \left.\sum_{j=1}^{n_{o p}} \int_{\mathcal{S}_{\mu}^{L_{j}}} d \tilde{\boldsymbol{\beta}}_{\mu}^{L_{j}} \otimes^{s} \boldsymbol{n}_{\mu}^{L_{j}} d \mathcal{S}_{\mu}=\boldsymbol{0}\right\},
\end{aligned}
$$

where, using the notation previously introduced, it has been considered the displacement jump fluctuation increment $d \tilde{\boldsymbol{\beta}}_{\mu}^{L_{j}}$ across the band $\mathcal{B}_{\mu}^{L_{j}}$, defined as: $\tilde{\boldsymbol{\beta}}_{\mu}^{L_{j}}=\left(\tilde{\boldsymbol{u}}_{\mu}^{+}\right)^{L_{j}}-$ $\left(\tilde{\boldsymbol{u}}_{\mu}^{-}\right)^{L_{j}}$, with $\left(\tilde{\boldsymbol{u}}_{\mu}^{+}\right)^{L_{j}}$ and $\left(\tilde{\boldsymbol{u}}_{\mu}^{-}\right)^{L_{j}}$ being the interpolated displacement fluctuations at each side of the band $L_{j}$ along the coordinate $\eta$, such as shown in the insert of Fig. 10.

The discrete space of virtual displacement actions $\mathscr{V}_{\mu}^{S h}$ is:

$$
\begin{aligned}
\mathscr{V}_{\mu}^{S h}= & \left\{\hat{\boldsymbol{u}}_{\mu}(\boldsymbol{y})=\sum_{k=1}^{n_{\text {nod }}} N_{\mu k}(\boldsymbol{y}) \hat{\boldsymbol{q}}_{\mu}^{k},\right. \\
& \text { such that } \int_{\Gamma_{\mu}^{h}} \hat{\boldsymbol{u}}_{\mu} \otimes^{s} \boldsymbol{\nu}_{\mu}^{h} d \Gamma_{\mu}=\mathbf{0} \\
& \text { and } \left.\sum_{j=1}^{n_{o p}} \int_{\mathcal{S}_{\mu}^{L_{j}}} \hat{\boldsymbol{\beta}}_{\mu}^{L_{j}} \otimes^{s} \boldsymbol{n}_{\mu}^{L_{j}} d \mathcal{S}_{\mu}=\boldsymbol{0}\right\},
\end{aligned}
$$


where, in concordance with the comment of the paragraph above, virtual variations of displacement jump across the band $\mathcal{B}_{\mu}^{L_{j}}$ are defined as $\hat{\boldsymbol{\beta}}_{\mu}^{i}=\hat{\boldsymbol{u}}_{\mu}^{+}-\hat{\boldsymbol{u}}_{\mu}^{-}$. The second kinematical constraint defining $\tilde{\mathscr{U}}_{\mu}^{S h}$ and $\mathscr{V}_{\mu}^{S h}$, extended along $\mathcal{S}_{\mu}^{L}$, ensures the satisfaction of identity (30) for the interpolated micro-scale strain fluctuations.

The kinematical constraints defined along $\Gamma_{\mu}^{h}$ in (79), and the first one in (82), can be seen as a specific kind of boundary condition defined on the RVE. Following the denomination introduced in our previous work (see [43]), we call it the Standard Boundary Conditions (SBC) and is the typical kinematical constraint defined in conventional semi-concurrent multi-scale models. The last constraint imposed along $\mathcal{S}_{\mu}^{L}$, in expression (82), is called the Non-Standard Boundary Conditions (NSBC). It can be seen as a boundary condition on the domain $\mathcal{B}_{\mu}^{L}$. Some remarks about these specific kinematics constraints are given:

- Standard Boundary Conditions (SBC). The model which strictly satisfies the kinematical constraint in (79) is called minimum kinematically constrained sub-model. However, kinematically more constrained sub-models can be defined, such as: Taylor, Linear or Periodic (see Toro et al. [43]).

The kinematical constraints introduced by different submodels play a very important role for adequately capturing the failure mechanism of the RVE leading to complete degradation of the material. The micro-scale failure mechanism is defined at $t_{S D}$. Thus, for $t<t_{S D}$, it should be carefully analyzed if the chosen sub-model inhibits the formation of some possible failure mechanism. The impediment to develop a failure mode due to spurious kinematical boundary conditions could produce a delay to detect macro-scale bifurcation and the possible identification of an alternative failure mechanism. This issue is strongly associated with the micro-cell size adopted for the analysis. Larger micro-cell sizes tend to decrease the effects introduced by boundary conditions, see the analysis presented in Toro et al. [43]. In general, smaller micro-cell sizes could be utilized for promoting correct failure mechanism if adequate boundary conditions are postulated.

- Non-Standard Boundary Conditions (NSBC). Once the macro-scale crack is activated and $d \boldsymbol{\beta} \neq 0$, the NSBC must be introduced in the model. The NSBC in the present finite element model are defined on the displacement fluctuation increment field $d \tilde{\boldsymbol{u}}_{\mu}(\boldsymbol{y})$ by constraining the vector $d \tilde{\boldsymbol{\beta}}_{\mu}^{L_{j}}=d\left(\tilde{\boldsymbol{u}}_{\mu}^{+}\right)^{L_{j}}-d\left(\tilde{\boldsymbol{u}}_{\mu}^{-}\right)^{L_{j}}$, along the localization band $\mathcal{B}_{\mu}^{L_{j}}$, to satisfy the second constraint in expression (82). A particular sub-model which trivially satisfies the NSBC consists in defining $d\left(\tilde{\boldsymbol{u}}_{\mu}^{+}\right)^{L_{j}}=d\left(\tilde{\boldsymbol{u}}_{\mu}^{-}\right)^{L_{j}} \equiv \boldsymbol{O}$ (i.e. fixing to zero the increment of displacement fluctuations of all nodes on the boundary of $\mathcal{B}_{\mu}^{L}$ ) and giving $d \tilde{\boldsymbol{\beta}}_{\mu}^{L_{j}} \equiv \boldsymbol{O}$. This sub-model is a generalization of the Taylor sub-model extended to the MMSp approach, and is chosen for solving the problems presented in next Sect. 4. Note that, even fixing to zero the displacement fluctuation increments into the opening localization bands, a non-homogeneous deformation takes place within such bands. In fact, this kind of deformation is induced by the macro-scale strains inserted by the operator $\mathcal{I}_{S}$.

Numerical treatment of the kinematical constraints. The spaces $\tilde{\mathscr{U}}_{\mu}^{R h}$ and $\tilde{\mathscr{U}}_{\mu}^{S h}$ (or alternatively, $\tilde{\mathscr{V}}_{\mu}^{R h}$ and $\tilde{\mathscr{V}}_{\mu}^{S h}$ ) are implemented by introducing the kinematical constraints (SBC and NSBC) as additional homogeneous linear equations between the degree of freedoms (d.o.f.'s) of the model.

Let us call $\left[d \tilde{\boldsymbol{q}}_{\mu}\right]$, with $\left[d \tilde{\boldsymbol{q}}_{\mu}\right] \in \mathbb{R}^{2 \times n_{\text {nod }}}$, the vector collecting the complete set of interpolation parameters for the displacement fluctuation increment field $\left(\left[d \tilde{\boldsymbol{q}}_{\mu}\right]^{T}=\right.$ $\left.\left[\left[d \tilde{\boldsymbol{q}}_{\mu}^{1}\right]^{T}, \ldots,\left[d \tilde{\boldsymbol{q}}_{\mu}^{n_{\text {nod }}}\right]^{T}\right]\right)$ of the finite element model. So, $n_{\text {dof }}=2 \times n_{\text {nod }}$ identifies the total number of d.o.f.'s of the model.

A direct mathematical treatment allows to write the SBC and NSBC as a system of $n_{d}$ homogeneous linearly independent equations as follows (see additional details of this point in Toro et al. [43]):

$\boldsymbol{H}\left[d \tilde{\boldsymbol{q}}_{\mu}\right]=\boldsymbol{0}, \quad \boldsymbol{H} \in \mathbb{R}^{\left(n_{d}, n_{d o f}\right) .}$

From the previous equation, the vector $\left[d \tilde{\boldsymbol{q}}_{\mu}\right]$ can be partitioned into two vectors of dimension $n_{d}$ and $n_{f}=n_{d o f}$ $-n_{d}$ :

$\left[d \tilde{\boldsymbol{q}}_{\mu}\right]=\left[\begin{array}{l}{\left[d \tilde{\boldsymbol{q}}_{\mu}\right]_{f}} \\ {\left[d \tilde{\boldsymbol{q}}_{\mu}\right]_{d}}\end{array}\right], \quad\left[d \tilde{\boldsymbol{q}}_{\mu}\right]_{f} \in \mathbb{R}^{n_{f}}, \quad\left[d \tilde{\boldsymbol{q}}_{\mu}\right]_{d} \in \mathbb{R}^{n_{d}}$,

where:

$\left[d \tilde{\boldsymbol{q}}_{\mu}\right]_{d}=\boldsymbol{L}\left[d \tilde{\boldsymbol{q}}_{\mu}\right]_{f}$,

being $L$ a matrix of coefficients which are defined according with the kinematical constraints, or discrete space, to be modeled. So, $\boldsymbol{L}=\boldsymbol{L}^{R h}$ identifies the coefficient matrix imposing the SBC constraint seen in (79), while $\boldsymbol{L}=\boldsymbol{L}^{S h}$ the matrix imposing both, SBC and NSBC, constraints seen in (82).

The RVE discrete vectors $\left[d \tilde{\boldsymbol{q}}_{\mu}\right]_{f}$ and $\left[d \tilde{\boldsymbol{q}}_{\mu}\right]_{d}$ are interpreted as the free and dependent partitions of the total d.o.f.'s, respectively.

Next, we identify the finite dimensional vector spaces corresponding to the vector parameters of displacement fluctuation increments: 
$\tilde{\mathscr{U}}_{\mu}^{R q}=\left\{\left[d \tilde{\boldsymbol{q}}_{\mu}\right] \in \mathbb{R}^{n_{d o f}}\right.$, and $\left.\left[d \tilde{\boldsymbol{q}}_{\mu}\right]=\left[\begin{array}{c}{\left[d \tilde{\boldsymbol{q}}_{\mu}\right]_{f}} \\ \boldsymbol{L}^{R h}\left[d \tilde{\boldsymbol{q}}_{\mu}\right]_{f}\end{array}\right]\right\}$,

and

$\tilde{\mathscr{U}}_{\mu}^{S q}=\left\{\left[d \tilde{\boldsymbol{q}}_{\mu}\right] \in \mathbb{R}^{n_{d o f}}, \quad\right.$ and $\left.\left[d \tilde{\boldsymbol{q}}_{\mu}\right]=\left[\begin{array}{c}{\left[d \tilde{\boldsymbol{q}}_{\mu}\right]_{f}} \\ \boldsymbol{L}^{S h}\left[d \tilde{\boldsymbol{q}}_{\mu}\right]_{f}\end{array}\right]\right\}$.

The finite dimensional vector spaces corresponding to the vector parameters of virtual actions, are defined as $\tilde{\mathscr{V}}_{\mu}^{R q} \equiv$ $\tilde{\mathscr{U}}_{\mu}^{R q}$ and $\tilde{\mathscr{V}}_{\mu}^{S q} \equiv \tilde{\mathscr{U}}_{\mu}^{S q}$.

Note that, fields $d \tilde{\boldsymbol{u}}_{\mu}$ obtained from the interpolation (64) with vectors $\left[d \tilde{\boldsymbol{q}}_{\mu}\right] \in \tilde{\mathscr{U}}_{\mu}^{R q}$, strictly satisfy that $d \tilde{\boldsymbol{u}}_{\mu} \in \tilde{\mathscr{U}}_{\mu}^{R h}$ (or alternatively, fields $d \tilde{\boldsymbol{u}}_{\mu}$ obtained from the interpolation (64) with vectors $\left[d \tilde{\boldsymbol{q}}_{\mu}\right] \in \tilde{\mathscr{U}}_{\mu}^{S q}$ strictly satisfy that $d \tilde{\boldsymbol{u}}_{\mu} \in$ $\left.\tilde{\mathscr{U}}_{\mu}^{S h}\right)$.

\subsubsection{Micro-scale variational equilibrium problem}

The variational discrete equilibrium equation in $\Omega_{\mu}^{h}$ for the MMRp homogenization procedure is implemented as follows: find $\left[\tilde{\boldsymbol{q}}_{\mu}\right] \in \tilde{\mathscr{U}}_{\mu}^{R q}$, such that:

$$
\int_{\Omega_{\mu}^{h}} \boldsymbol{\sigma}_{\mu} \cdot \hat{\varepsilon}_{\mu} d \Omega=\left[\hat{\boldsymbol{q}}_{\mu}\right]^{T} \boldsymbol{F}_{\mu}^{i n t}=0 \quad, \quad \forall\left[\hat{\boldsymbol{q}}_{\mu}\right] \in \mathscr{V}_{\mu}^{R q},
$$

where the vector of internal forces $\boldsymbol{F}_{\mu}^{i n t}\left(\left[\tilde{\boldsymbol{q}}_{\mu}\right]\right)$ is:

$\boldsymbol{F}_{\mu}^{\text {int }}=\Lambda_{e_{\mu}=1}^{\text {nelem }_{\mu}} \int_{\Omega_{\mu}^{e_{\mu}}} \boldsymbol{B}_{\mu}^{e_{\mu} T} \boldsymbol{\sigma}_{\mu}^{e_{\mu}}\left(\varepsilon_{\mu}^{e_{\mu}}\right) d \Omega_{\mu}$.

The stresses $\sigma_{\mu}^{e_{\mu}}$ in (90) are evaluated with the corresponding constitutive relations associated with every micro-scale component. Also, the micro-scale strains $\varepsilon_{\mu}^{e_{\mu}}$ are determined by (65) and (80). The assembling operation of the internal force vector (90) takes into account the total number of finite elements $\left(\right.$ nelem $\left._{\mu}\right)$ in $\Omega_{\mu}^{h}$, including the CST elements of the micro-scale bands.

Expression (89) is the proposed discrete implementation to approximate the micro-scale equilibrium (28). A numerical strategy for solving (89) has been presented in in Toro et al. [43].

Observe that taking the spaces $\tilde{\mathscr{U}}_{\mu}^{S q}$ and $\mathscr{V}_{\mu}^{S q}$ in Eq. (89) instead of $\tilde{\mathscr{U}}_{\mu}^{R q}$ and $\mathscr{V}_{\mu}^{R q}$, the variational equilibrium equation and the numerical treatment given to the MMSp is obtained.

The connection between the variational equilibrium equation (89), considering the spaces $\tilde{\mathscr{U}}_{\mu}^{S q}$ and $\mathscr{V}_{\mu}^{S q}$, and the equilibrium Eq. (40) for a micro-cell with cohesive surfaces is shown through the following identity, which holds for the present micro-scale finite element model using the CST bands:

$$
\begin{aligned}
& \int_{\Omega_{\mu}^{h}} \boldsymbol{\sigma}_{\mu} \cdot \hat{\varepsilon}_{\mu} d \Omega=\int_{\Omega_{\mu}^{h} \backslash \mathcal{B}_{\mu}} \boldsymbol{\sigma}_{\mu} \cdot \hat{\varepsilon}_{\mu} d \Omega \\
& \quad+\sum_{i=1}^{n_{c}} \int_{\mathcal{S}_{\mu}^{i}} \boldsymbol{T}_{\mu}^{i} \cdot \hat{\boldsymbol{\beta}}_{\mu}^{i} d \mathcal{S}=0, \quad \forall\left[\hat{\boldsymbol{q}}_{\mu}\right] \in \mathscr{V}_{\mu}^{S h},
\end{aligned}
$$

where, the intermediate identity is obtained by partitioning the integral into two disjoint sets $\Omega_{\mu}^{h} \backslash \mathcal{B}_{\mu}$ and $\mathcal{B}_{\mu}$, and applying the divergence theorem to the integral in $\mathcal{B}_{\mu}$. Here, it is recalled that we are assuming: $\ell_{\mu}^{i} \rightarrow 0$.

Furthermore, in (91), we define: $\boldsymbol{T}_{\mu}^{i}=\boldsymbol{\sigma}_{\mu}\left(\boldsymbol{n}_{\mu}^{i}\right)^{+}$. Therefore, the second term in the middle expression of (91) can be interpreted as the virtual internal power due to cohesive tractions $\boldsymbol{T}_{\mu}^{i}$ acting on the band $\mathcal{B}_{\mu}^{i}$.

Note that the variational equilibrium equation (91), arising from the present finite element model at the micro-scale, resembles the expression (40) which has been defined for a model with a strong discontinuity kinematics.

If strain localization is not observed in the $i$-th band (CST elements), the vectors $\boldsymbol{\beta}_{\mu}^{i}$, as well as $\tilde{\boldsymbol{\beta}}_{\mu}^{i}$, can be assumed very small. Then:

$\lim _{\ell_{\mu}^{i} \rightarrow 0} \frac{\beta_{\mu}^{i}}{\ell_{\mu}^{i}} \approx \mathcal{O}\left(\varepsilon_{\mu_{\Omega_{\mu} \backslash \mathcal{B}_{\mu}}}\right)$,

where $\varepsilon_{\mu_{\Omega_{\mu} \backslash \mathcal{B} \mu}}$ is the strain in the zone adjacent to the band $\mathcal{B}_{\mu}^{i}$. Thus, the internal work in $\mathcal{B}_{\mu}^{i}$ is:

$\int_{\mathcal{B}_{\mu}^{i}} \boldsymbol{T}_{\mu}^{i} \cdot \boldsymbol{\beta}_{\mu}^{i} d \mathcal{S} \approx 0$.

However, once the strain localization process starts in the $i$-th band, the relative displacement between nodes across the bands can no longer be assumed negligible. The relative displacements across the band take the role of displacement jumps. Then:

$\lim _{\ell_{\mu}^{i} \rightarrow 0} \frac{\beta_{\mu}^{i}}{\ell_{\mu}^{i}} \rightarrow \infty$

and the internal work in $\mathcal{B}_{\mu}^{i}$ is no longer necessarily small. So, the kinematics of this finite element behaves like a strong discontinuity kinematics, and the band elements introduce a similar response to conventional cohesive interface elements.

This finite element technique can be seen as a regularization procedure, using continuum displacement interpolations, of problems displaying strong discontinuities. A 
similar technique for mono-scale analysis has been reported in Manzoli et al. [49] and Oliver et al. [31].

\subsubsection{Degenerated CST elements with strain localization for simulating the RVE failure: pros and cons}

The computational technique developed for the micro-scale analysis has two remarkable features:

(a) Contrarily to the observed response when classical interface cohesive models are used, CST band elements described with continuous damage model and identical elastic behavior to that of the bulk material do not feature spurious stress oscillations.

(b) This approach, coupled with an Impl-Ex constitutive integration scheme (c.f. Oliver et al. [48]), is very robust. The only non-standard step in the numerical implementation of the RVE finite element model is related to the mesh generation, in the sense that, arbitrary meshes ought to receive a pre-treatment to introduce CST band elements between all finite element edges of the original mesh.

In turn, it can be noted two serious limitations for modeling micro-structural failure mechanisms with this technique: (i) arbitrary crack paths cannot be captured, the crack path must necessarily follow the element edges of the predefined finite element mesh, and (ii) severe numerical locking arises for capturing shear bands when non-aligned CST elements are taken. The last deficiency can be alleviated, in part, by using damage-type models to characterize the CST elements.

\subsection{A tracking algorithm for determining the macro-scale crack path}

The problem of finding the geometrical position of $\mathcal{S}^{h} \subset \Omega^{h}$ at the macro-scale, is an important issue of the finite element implementation. As mentioned above, the crack path must be precisely determined before inserting the enriching (DDM) mode for elements in state 2 .

To achieve this objective, first it is defined a new field $\varrho(\boldsymbol{x})$ at the macro-scale domain $\Omega^{h}$ as follows:

$\varrho(\boldsymbol{x})=\frac{1}{\left|\Omega_{\mu}\right|} \sum_{i=1}^{n_{c}} \int_{\mathcal{B}_{\mu}^{i}} r_{\mu} d \mathcal{B}_{\mu}$

So, $\varrho$ is the average value in the cell of the micro-scale strainlike internal variable $r_{\mu}$ defined in Box 3. Since $r_{\mu}$ represents a maximum strain measure during the loading history, $r_{\mu}$ becomes very large, proportional to $1 / \ell_{\mu}^{i}$, in those bands displaying strain localization. However, the integral in (95) is not necessarily zero when $\ell_{\mu}^{i} \rightarrow 0$ due to the area of the band $\left|\mathcal{B}_{\mu}^{i}\right|$ goes to zero.

At the instant of macro-scale bifurcation, at $t=t_{N}, \varrho(x)$ quickly increases, because a sufficient large number of microscale bands are subjected to a loading processes. Therefore, the macro-scale regions where the material is reaching the instability condition are characterized by large values of $\varrho$. Contrarily, $\varrho$ is very small in regions where the inelastic regime has not yet been achieved. We use this property of the field $\varrho$ to predict the geometrical position of the evolving discontinuity surface $\mathcal{S}^{h}$.

Let us take, at the macro-scale finite element domain $\Omega^{h}$, the space of functions $\mathscr{L}$ coinciding with the standard bilinear interpolation field $H^{1}\left(\Omega^{h}\right)$ of the quadrilateral finite element, and the space of piece-wise constant functions, $\mathscr{C}\left(\Omega^{h}\right)$ :

$\mathscr{C}\left(\Omega^{h}\right):=\left\{\psi(\boldsymbol{x}) \mid \psi(\boldsymbol{x})=\mathrm{constant}\right.$ in $\left.\Omega^{e}\right\}$.

The geometrical position of $\mathcal{S}^{h}$ intersecting the finite element mesh can be detected by solving two successive problems, described in the following items as steps 1 and 2. Every step involves the projection of discontinuous fields onto $\mathscr{L}$.

- Step 1: Find the field $\chi(x) \in \mathscr{L}$, such that:

$\int_{\Omega^{h}} \hat{\chi}(\chi-\varrho(\boldsymbol{x})) d \Omega^{h}=0, \quad \forall \hat{\chi} \in \mathscr{L}$.

Then, $\chi(\boldsymbol{x})$ can be seen as a smoothing of $\varrho$. The quadrature rule to integrate (97) follows the similar scheme defined in Sect. 3.1.

- Step 2: Find the directional derivative of $\chi, \xi_{c}^{h} \in \mathscr{C}$, along the direction given by the vector $\omega$ :

$\xi_{c}^{h}\left(\Omega^{e}\right)=\nabla_{x} \chi\left(\boldsymbol{x}_{S}^{e}\right) \cdot \boldsymbol{\omega}\left(\boldsymbol{x}_{S}^{e}\right)$,

where the vector field $\omega$ is a sufficiently smooth extension to $\Omega^{h}$ of the normal vector to $\mathcal{S}^{h}$ whose location has to be found.

Finally, the so-called crack-path field $\kappa \in \mathscr{L}$ is determined as the smoothing of $\xi_{c}^{h}$. It is evaluated according to the projection:

$\int_{\Omega} \hat{\kappa}\left(\kappa-\xi_{c}^{h}\right) d \Omega^{h}=0, \quad \forall \hat{\kappa} \in \mathscr{L}$.

The manifold $\Pi(\boldsymbol{x})$ is defined as the zero level set of the crack-path field $\kappa$ :

$\boldsymbol{x} \in \Pi \quad$ iff $\quad \kappa(\boldsymbol{x})=0$.

It can be shown (see Oliver et al. [46]) that the discontinuity surface satisfies $\mathcal{S}^{h} \subset \Pi$. Therefore, the problem of 
determining $\mathcal{S}^{h}$ is reduced to finding the set $\Pi$ given by the algebraic procedure defined in equations (97)-(100).

\section{Numerical assessment}

In a recent contribution by the authors (Toro et al. [44]), some important issues of the present multi-scale model, such as the sensitivity of macro-scale solutions to tortuosity parameter changes, have been analyzed. Here, additional studies are addressed to further validate the implementation of the multiscale methodology.

With this goal in mind, concrete fracture problems are solved by considering the heterogeneities observed at the meso-scale. Hence, the numerical simulations take into account two-scales of analysis: a) the structural scale and b) the meso-structural scale at lengths of the order of the aggregate sizes. The interaction effects between aggregates and cement-based matrix, mainly those related to fracture, are included in the analysis.

First, in Sect. 4.1, we describe the meso-structure model developed for concrete. We define the constituents considered at the meso-scale and their possible interactions. In Sect. 4.2, through a simple uniaxial macroscopic stretching test, we assess the sensitivity of structural results to changes of the mesoscopic cell sizes and finite element sizes modeling the cell. Finally, in Sect. 4.3 we simulate a double notch beam bending concrete test. Results are computed with several multi-scale models and are validated by comparing with Direct Numerical Simulation (DNS) solutions.

\subsection{Concrete failure modeling at the meso-scale}

At the meso-scale, three main constituents can be recognized in concrete: cementitious matrix, aggregates and the Interface Transition Zone matrix/aggregate (ITZ). The ITZ plays an important role in the degradation mechanism of this composite. Failure generally initiates at the ITZ, which can be considered as the weakest link for concrete of low to moderate strengths, with the posterior propagation of cracks across the matrix. In this kind of concrete, trans-aggregate fracture is almost never observed. Then, in the present model, the aggregates are assumed to behave like an elastic material with no degradation at all.

The meso-scale model for concrete failure developed in this Section follows very closely the approximations reported in Carol et al. [50] and Unger and Eckardt [51].

Meso-structure model. Representation of an idealized twodimensional composite model at the meso-scale is performed by selecting, in the plane of analysis, different shape and size particles. The particles represent the aggregates, and they are randomly distributed into a mesoscopic square cell of different sizes, depending on the tests shown in the following. These aggregates are assumed to have shapes similar to irregular convex polygons and sizes which are determined from a predefined grading curve $(83 \%$ of particles with sizes between $3[\mathrm{~mm}]$ to $4[\mathrm{~mm}]$, and $17 \%$ of particles with sizes between $4[\mathrm{~mm}]$ to $5[\mathrm{~mm}])$. The total volume fraction of aggregates results $20.7 \%$. Aggregates distribution is such that they do not intersect the RVE boundaries.

According with the finite element procedure described in Sect. 3.2, the meso-scale model is designed as shown in Fig. 12. CST elements like-bands are inserted along the ITZ, as well as along all the edges of the finite elements, in the original mesh, covering the matrix domain. All these CST elements are characterized with the damage model described in Box 3.

Material parameters of the meso-scale model. Material parameters of the mesoscopic model are shown in Table 1. These data have been taken from Unger and Eckardt [51] with all the elastic moduli increased $10 \%$. The parameters defined in [51], such as peak stresses and fracture energies of constituents, are considered a very rough estimation for simulating the concrete beam test in Sect. 4.3 Note that the experimental work (Bocca et al. [52]) taken for validating the numerical results in Sect. 4.3, reports an overall Young's modulus $E=27 .[G P a]$ and an overall fracture energy $G^{f}=100 \cdot[N / m]$.

\subsection{Multi-scale model sensitivity analysis}

By using a simple macro-structural test, linked to complex mesoscopic cell models such as defined in the previous subSection, we analyze the sensitivity of the multi-scale model response to changes of cell size and finite element size of the meso-scale model. The analysis is addressed to evaluate the sensitivity of:

(i) the crack pattern obtained at the meso-scale level;

(ii) the macro-scale crack orientation obtained with Eq. (76);

(iii) the homogenized traction-separation law at the macroscale.

We study a strip of size $125 \mathrm{~mm} \times 125 \mathrm{~mm}$ and thickness $100 \mathrm{~mm}$ undergoing uniaxial stretching, such as depicted in Fig. 13. Two analyses are performed with this strip:

- Case (a): three specimens stretched in the directions $\alpha=$ 0 [deg], $\alpha=30$ [deg] and $\alpha=45[\mathrm{deg}]$, respectively, are solved with three mesoscopic cell sizes of $25 \mathrm{~mm}$ $\times 25 \mathrm{~mm}, 50 \mathrm{~mm} \times 50 \mathrm{~mm}$ and $75 \mathrm{~mm} \times 75 \mathrm{~mm}$. In this case, the size of the finite elements are similar in the three cells; 


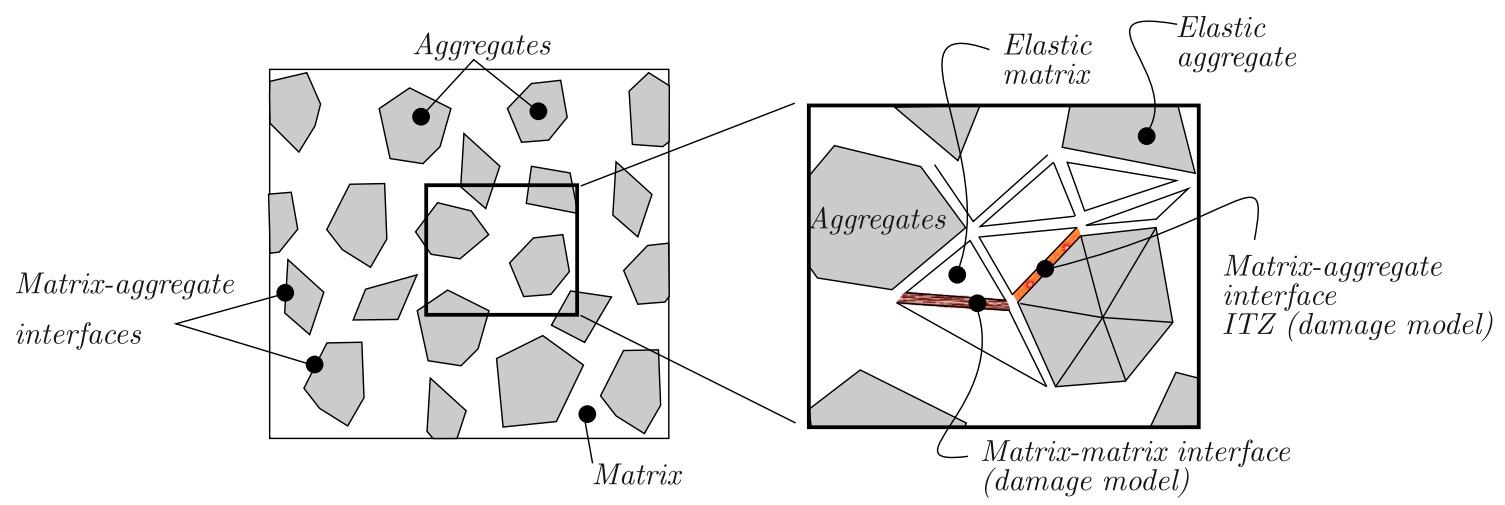

(a)

(b)

Fig. 12 Mesoscopic concrete model

Table 1 Material properties for the meso-structure depicted in Fig. 12

\begin{tabular}{lllll}
\hline & $E_{\mu}[G P a]$ & $v_{\mu}$ & $\sigma_{\mu}^{u}[M P a]$ & $G_{\mu}^{f}[\mathrm{~N} / \mathrm{m}]$ \\
\hline Elastic matrix & 20.4 & 0.18 & - & - \\
Elastic aggregate & 40.8 & 0.18 & - & - \\
Interface matrix-matrix & 20.4 & 0.18 & 2.6 & 140. \\
ITZ matrix-aggregate & 20.4 & 0.18 & 1.3 & 70.
\end{tabular}

Parameters defined are: Young's modulus, $E_{\mu}$, Poisson ratio $v_{\mu}$, ultimate tensile stress $\sigma_{\mu}^{u}$ and fracture energy $G_{\mu}^{f}$

- Case (b): the specimen stretched in the direction $\alpha=$ 0 [deg] of the case a) is solved with the cell size $50 \mathrm{~mm} \times$ $50 \mathrm{~mm}$, using three meshes whose finite element sizes are: coarse (Mesh I), medium (Mesh II) and fine (Mesh III).

In all cases, the kinematical constraints imposed on the micro-cells are: (a) minimal kinematical constraints and displacement fluctuation increments of the four cell vertices fixed to zero for the SBC; (b) a Taylor sub-model for the NSBC.

Note that, in all specimens, the micro-cells are modeled with their edges parallel to the horizontal and vertical directions. So, the expected failure pattern developed in the three specimens intersects the micro-cell edges at different angles, depending on the stretching direction at the macro-scale. Therefore, from this analysis, it is also possible to assess the effect (if any) induced by the SBC and NSBC for capturing different meso-scale failure patterns.

\section{Case (a) sensitivity analysis to changes of cell sizes}

The failure modes captured at the meso-scale in the three specimens, using different cell sizes, are analyzed in Fig. 13. This Figure displays the deformed configurations of the mesoscopic cells at the end of analysis. In deep red are shown the CST finite elements which are opening at the end of analysis, defining the strain localization domain $\mathcal{B}_{\mu}^{L}$. In light blue are depicted the CST finite elements which have been opening during the pre-critical regime, but are closing at the post-critical regime.

Table 2 presents additional sensitivity results. The angles forming the macro-scale normal vector $\boldsymbol{n}^{e}$ with the horizontal direction are shown there. These vectors are determined with equation (76). The tortuosity parameters, evaluated with Eq. (17), are also presented in the last column of the Table. Note the small dispersion of $\theta$ obtained in different instances, as well as the close coincidence between the resulting direction of $\boldsymbol{n}^{e}$ and the stretching direction.

Figure 14a displays the structural force $(F)$ vs. macroscale displacement $(\delta)$ curves for all instances. Again, a close coincidence can be observed in all these cases. As expected, a convergence is observed as the cell size is increased. In turn, Fig. 14b presents, for the mesoscopic cell $50 \mathrm{~mm} \times 50 \mathrm{~mm}$ and stretching direction $30[\mathrm{deg}]$, the normal component of macro-scale stress $\left(\sigma_{n n}\right)$ versus macro-scale strain $\left(\varepsilon_{n n}\right)$ during the pre-bifurcation regime $\left(t \leq t_{S D}\right)$. In the same Figure, it is also plotted the normal component of the macro-scale traction $\left(T_{n}\right)$ vs. the normal component of the macro-scale displacement jump $\left(\beta_{n}\right)$ during the post-bifurcation regime $\left(t \geq t_{S D}\right)$. Because both responses, see the two plots in Fig. 14b, are obtained with different homogenization procedures (MMRp and MMSp), it is important to analyze the continuity at $t=t_{S D}$ provided by them. This a very important issue. The continuity of responses with both procedures of the present multi-scale model is a result of introducing the tortuosity parameter $\theta$ into the insertion operators $\mathcal{I}$, as well as, in the traction homogenization formula (39), see additional discussion about this aspect of the model in [44].

From this analysis, it can be concluded that the cell with $25 \mathrm{~mm} \times 25 \mathrm{~mm}$ provides results which are considered as objective for the present concrete mesoscopic model. Furthermore, the macro-scale response, including the traction-separation law, the evaluation of the normal vector $\boldsymbol{n}^{e}$ and the tortuosity parameter $\theta$, are not significantly sensitive to the mesoscopic cell size for cells larger than $25 \mathrm{~mm} \times 25 \mathrm{~mm}$. 

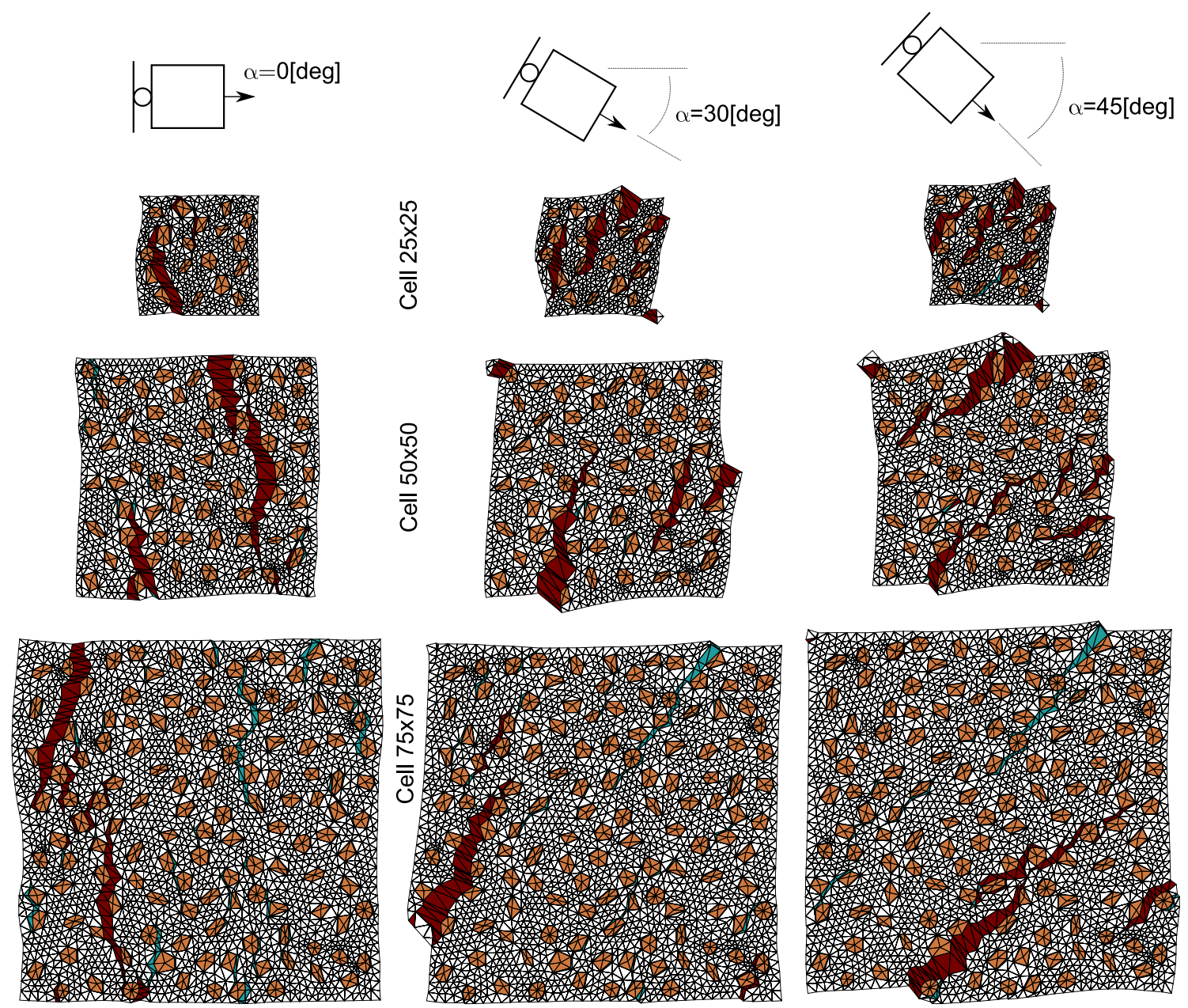

Fig. 13 Sensitivity analysis to changes of the cell size. Mesoscopic cell deformed configurations at the end of analysis depicting the failure modes for different macro-scale stretching orientation and cell sizes. (Color figure online)

Table 2 Macro-scale normal angle $\left(\boldsymbol{n}^{e}\right)$ and tortuosity parameter obtained with different mesoscopic cell sizes for the three specimens stretched at angles $\alpha$

\begin{tabular}{llll}
\hline $\begin{array}{l}\text { Mesoscopic cell } \\
\text { size }(\mathrm{mm})\end{array}$ & $\begin{array}{l}\text { Principal } \\
\text { stretching angle } \\
\alpha[\mathrm{deg}]\end{array}$ & $\begin{array}{l}\text { Angle of the } \\
\text { macro-scale normal } \\
\boldsymbol{n}^{e}[\mathrm{deg}]\end{array}$ & $\begin{array}{l}\text { Tortuosisty } \\
\text { parameter } \\
\theta\end{array}$ \\
\hline $25 \times 25$ & 0 & 5 & 0.89 \\
$25 \times 25$ & 30 & 26 & 0.87 \\
$25 \times 25$ & 45 & 50 & 0.86 \\
$50 \times 50$ & 0 & 4 & 0.88 \\
$50 \times 50$ & 30 & 33 & 0.82 \\
$50 \times 50$ & 45 & 43 & 0.84 \\
$75 \times 75$ & 0 & 0 & 0.86 \\
$75 \times 75$ & 30 & 26 & 0.85 \\
$75 \times 75$ & 45 & 43 & 0.86 \\
\hline
\end{tabular}

\section{Case (b) sensitivity analysis to changes of finite element} mesh sizes

Using the same structural problem of case a) and a microcell of $50 \mathrm{~mm} \times 50 \mathrm{~mm}$ stretched in the horizontal direction, we analyze the results obtained with three different finite element meshes: Mesh (I) with 4190 finite elements, Mesh (II) with 7964 finite elements and Mesh (III) with 17970 finite elements, respectively. Fig. 15a displays the crack pattern 

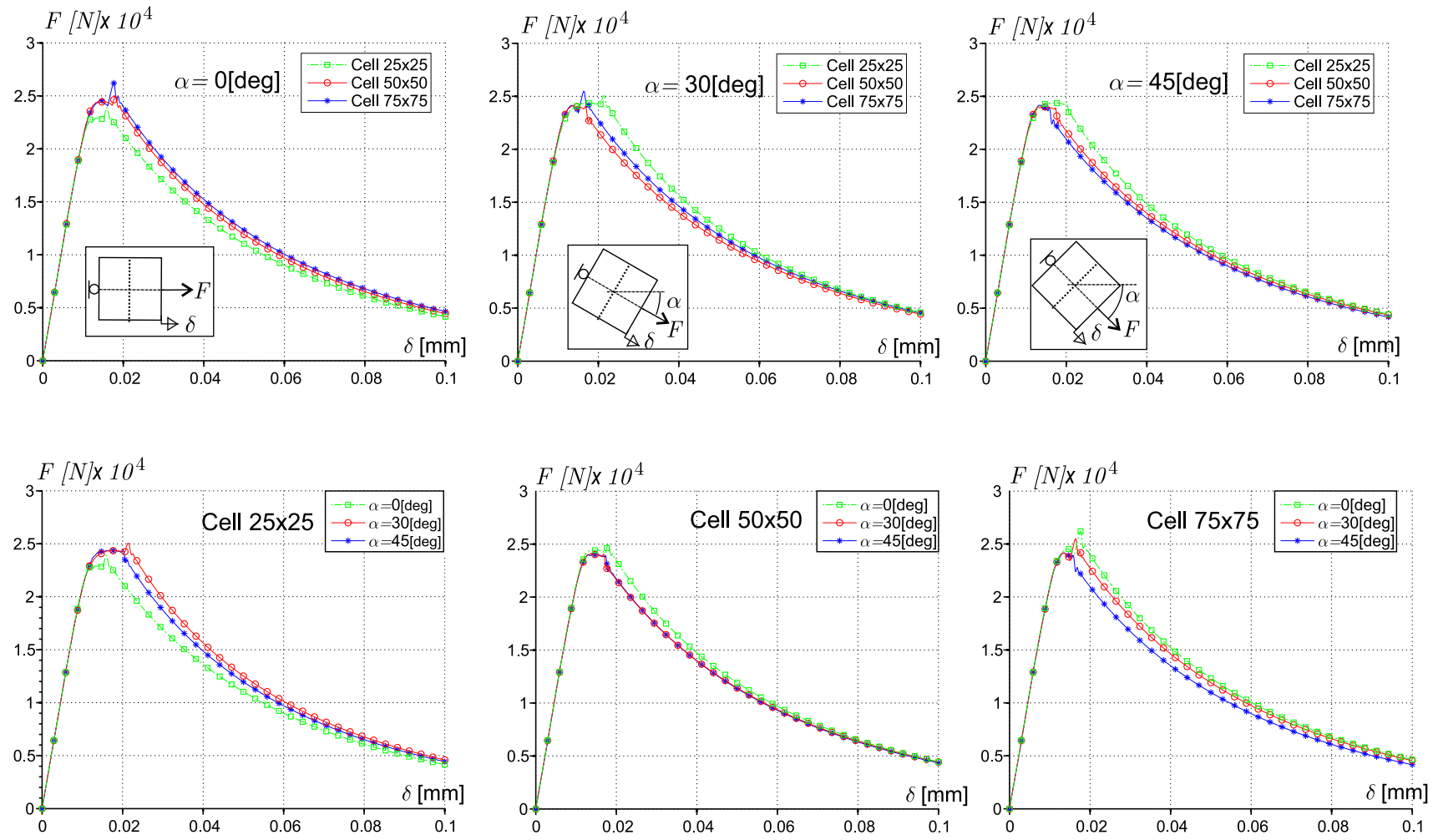

(a)
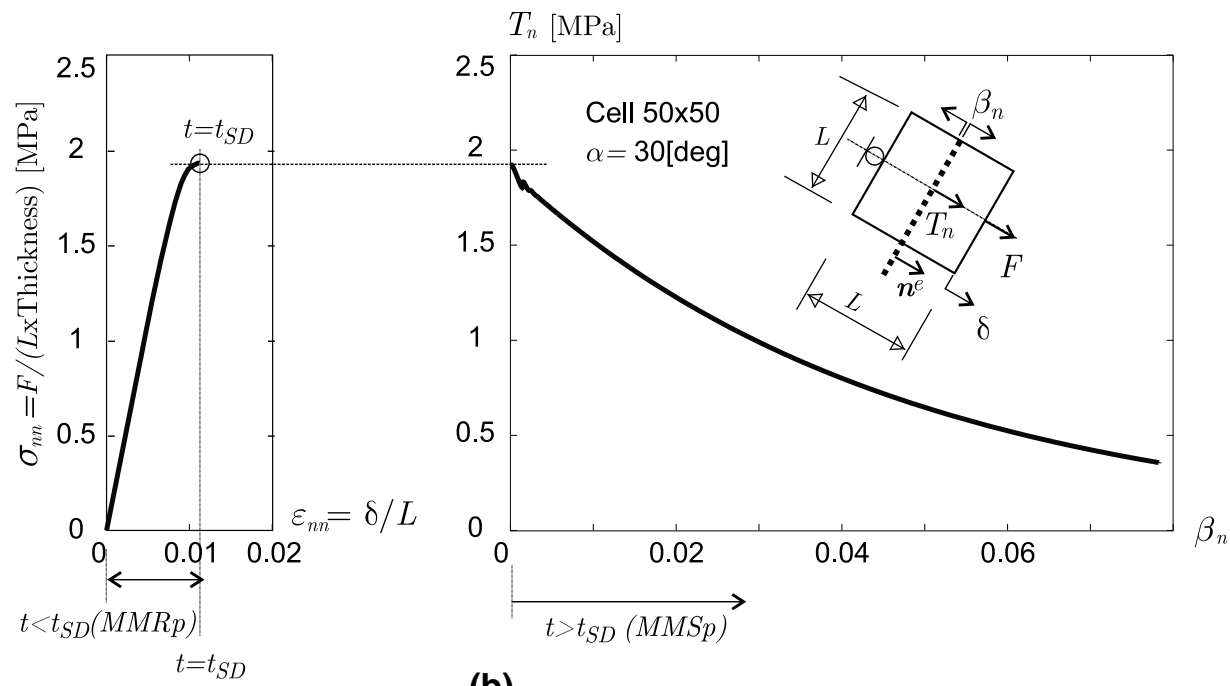

(b)

Fig. 14 Sensitivity analysis to changes of cell sizes. a Load vs. displacement plots for the three specimens using three cell sizes: $25 \mathrm{~mm}$ $\times 25 \mathrm{~mm}, 50 \mathrm{~mm} \times 50 \mathrm{~mm}$ and $75 \mathrm{~mm} \times 75 \mathrm{~mm}$; b Macro-scale stressstrain curve ( $\sigma_{n n}$ vs. $\varepsilon_{n n}$, subscript indicates components in the direction

observed on each mesoscopic cell at the end of analysis, as well as the tortuosity parameters and the angles between the normal vector $\boldsymbol{n}^{e}$ and the horizontal direction, obtained in each case. of $\boldsymbol{n}^{e}$ ) during the pre-bifurcation regime and macro-scale tractionseparation law $\left(T_{n}\right.$ vs. $\left.\beta_{n}\right)$ during the post-bifurcation regime captured with the micro-cell of $50 \mathrm{~mm} \times 50 \mathrm{~mm}$ and stretching direction: 30 [deg]

From this analysis, it can be concluded that the evaluations of the tortuosity parameter and the macro-scale normal vector $n^{e}$ are not sensitive to the finite element mesh size. 


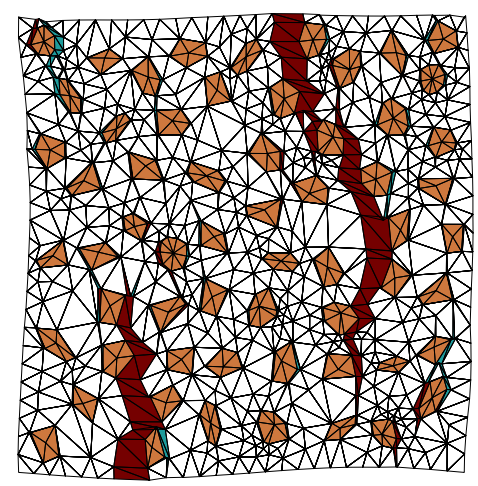

$\operatorname{Mesh}(I): 4190 \mathrm{FE}$

$\theta=0.86$

Angle of $\boldsymbol{n}^{e}=1[\mathrm{deg}]$

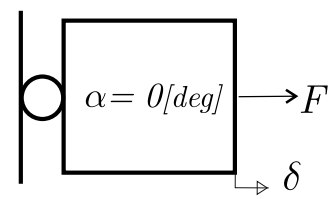

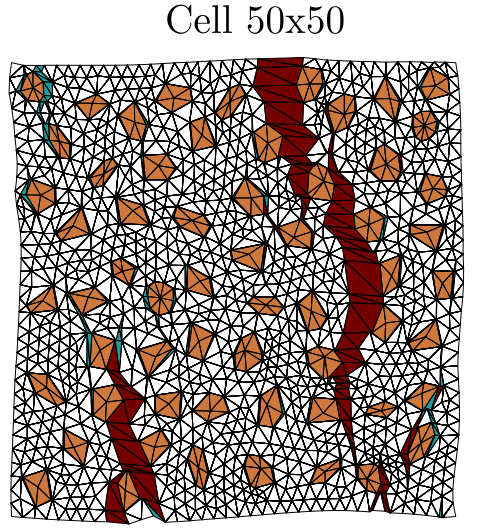

$$
\begin{gathered}
\text { Mesh (II): } 7694 \text { FE } \\
\quad \theta=0.88 \\
\text { Angle of } \boldsymbol{n}^{e}=4[\mathrm{deg}]
\end{gathered}
$$

(a)

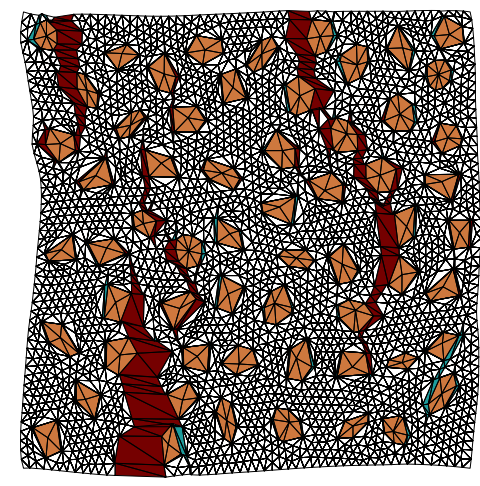

$$
\begin{gathered}
\text { Mesh (III):17970 FE } \\
\theta=0.84
\end{gathered}
$$

Angle of $\boldsymbol{n}^{e}=4[\mathrm{deg}]$

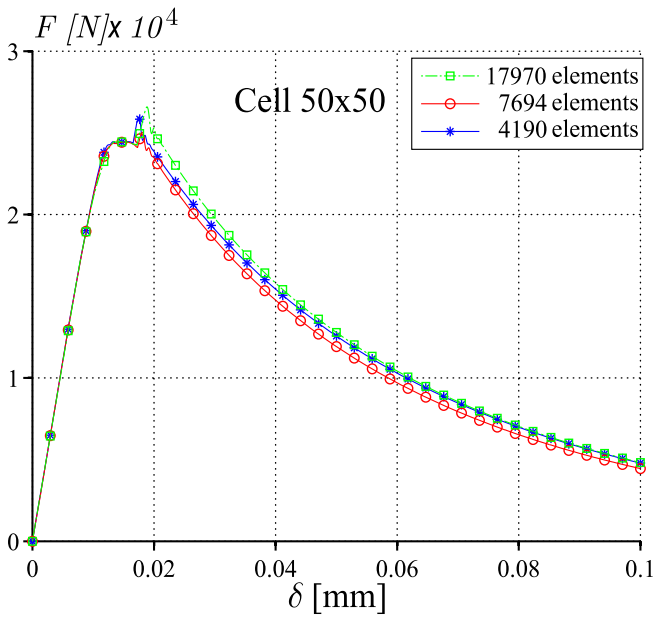

(b)

Fig. 15 Sensitivity analysis to changes of the micro-scale finite element mesh size. Solutions obtained with meshes (I), (II) and (III), respectively. a Failure mechanisms observed at the end of analysis, tor-

\subsection{Four point shear concrete specimen}

The beam with the loading system displayed in Fig. 16a is numerically simulated assuming plane stress condition. In the Figure, units of length are given in millimeters.

This beam corresponds to one of the 27 concrete specimens reported by Bocca et al. [52] ( $b=200 \mathrm{~mm}$ and $c / b=$ 0.8 ), which, according with these authors, have been conceived to assess the shear effects on the process of cracking.

The beam is here modeled and simulated using the multiscale formulation developed in this work (denoted MS model) and results are compared and validated with solutions obtained using three different Direct Numerical Simulation (DNS) models, where the meso-structure is particularly regarded. The reason of solving three DNS problems is to assess the high sensitivity and dispersion of the numerical tuosity parameters and the angles between the normal vector direction $\left(\boldsymbol{n}^{e}\right)$ and the horizontal axis; b Structural force $(F)$ versus macro-scale displacement $(\delta)$ curves. (Color figure online)

results that we have detected in this specific problem due to changes of aggregate distribution in the zones close to the notches.

MS finite element model. The finite element mesh adopted for the MS model at the macro-scale is shown in Fig. 16a. A multi-scale approach is only used in the central zone of the beam, depicted in blue. In the remaining part of the specimen, depicted in gray, an elastic mono-scale region is assumed. The homogenized elastic tensor $C^{\text {hom }}$ of the multiscale model is taken for simulating the elastic response of the mono-scale model. The meso-scale is modeled with the cell depicted in Fig. 16b.

First, we compare and discuss the numerical solutions obtained with the DNS and MS models. In the last case, 


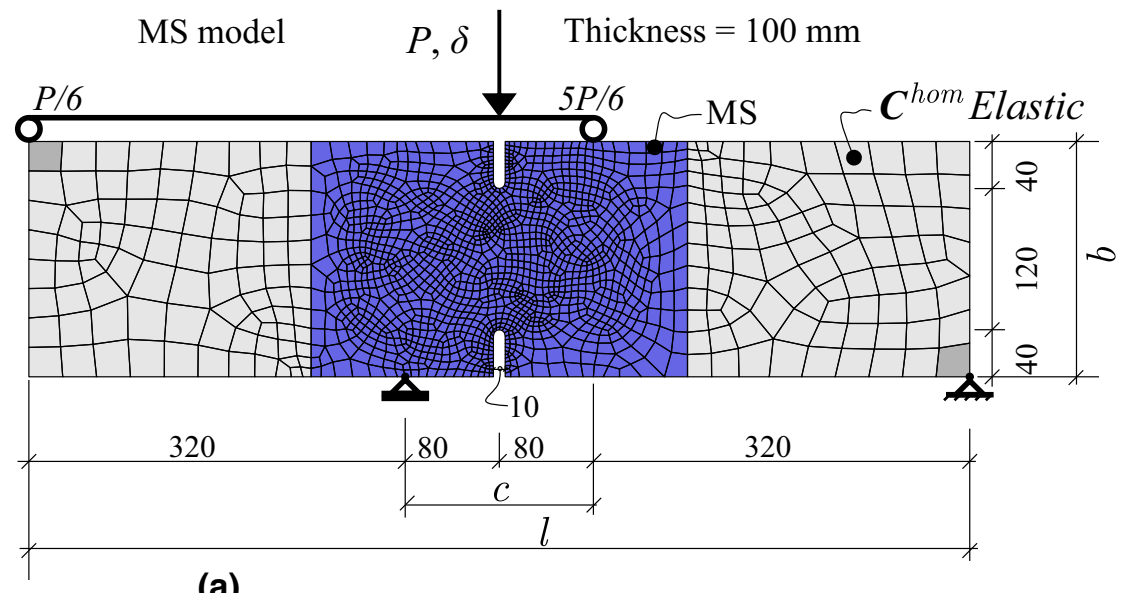

(a)
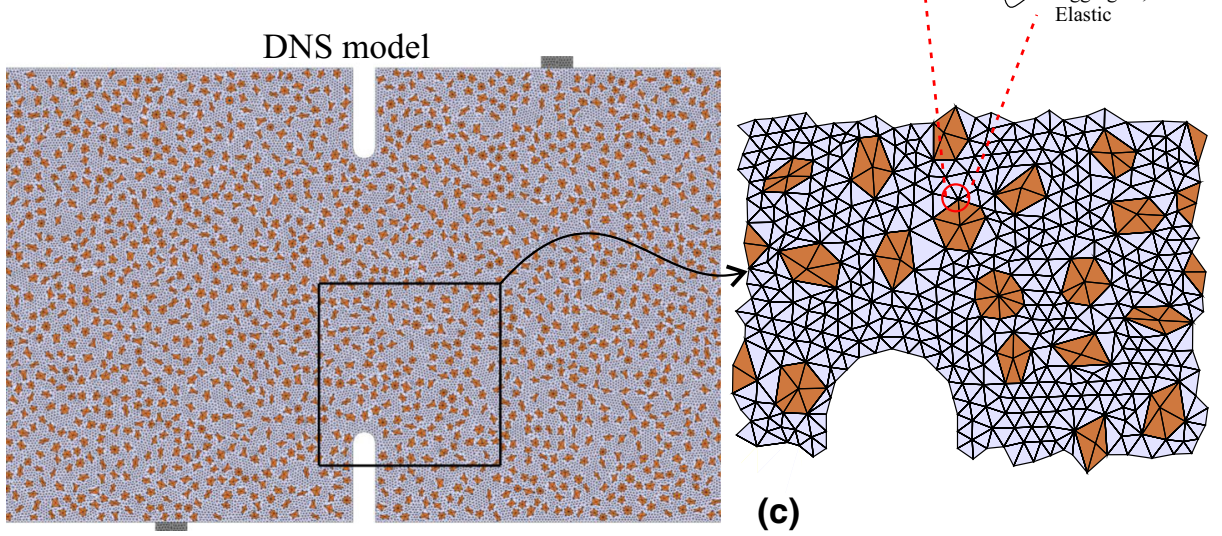

(c)

Fig. 16 Four point shear concrete specimen. a Geometry and multi-scale (MS) finite element model (dimension in millimeters); b mesoscopic cell; $\mathbf{c}$ one (of the three) DNS model. (Color figure online)

a micro-cell subjected to the following types of boundary conditions (BC's) are considered:

\section{BC's type I}

(a) SBC: minimum kinematical constraints with zero displacement fluctuations at the four vertices of the cell;

(b) NSBC: Taylor sub-model.

\section{BC's type II:}

(a) SBC: periodic;

(b) NSBC: Taylor sub-model.

In the final part of this Section, we compare the numerical solutions obtained with the two described boundary conditions of the MS models.

DNS finite element models. Figure 16c shows one of the three DNS models. The three DNS finite element models are built using an identical approach to that adopted for modeling the meso-scale cell described in Sect. 4.1. So, a random distribution of aggregates is inserted into the central part of the beam preserving the same volume fraction of aggregates. CST elements forming thin bands are placed between the edges of elements in the original mesh. The three meshes of the DNS models use similar finite element sizes.

\section{Discussion of results}

(a) Morphology of the crack patterns and structural force vs. displacement curves.

Figure 17 displays the evolution of the fracture process obtained with one of the three DNS models. In deep red are shown the bands formed by the CST finite elements which are opening at the end of the analysis. They define the crack paths leading to the structural collapse. In light red are depicted the bands which have been opening during part of the loading process but finally close. The complete fracture process can be described through two different stages. First, a large number of parallel small cracks (lengths of the order of aggregate sizes) are dispersed. They are approximately orthogonal to the principal stress directions (depicted with a cross). These meso-scale cracks are nucleated in almost the complete central region between both notches of the beam (see Fig. 17a, b). Notably, in a second stage, some of these cracks, close to the notches, coalesce in a different direction, forming two competing macro-scale principal cracks. The propagation of 


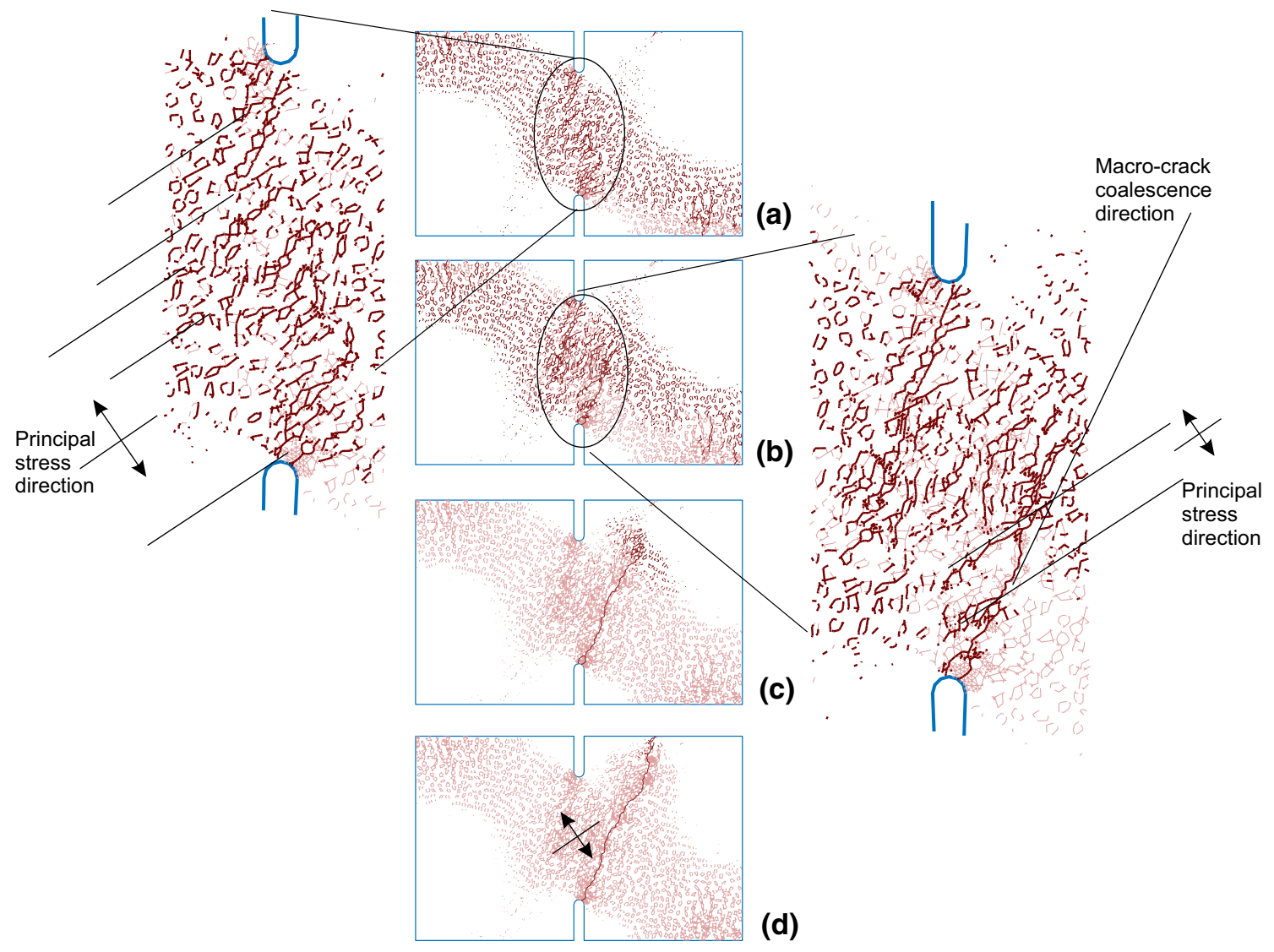

Fig. 17 Four point shear concrete specimen. a-d Sequence of pictures displaying the crack pattern evolution obtained with a DNS model (bands formed by opening CST finite element, at the end of the analysis,

one of these macro-scale cracks finally leads to the structural collapse.

As it is observed in the following, the present multi-scale model captures with acceptable accuracy this rather complex fracture process.

Figure 18a compares several numerical structural responses in terms of the load $P$ versus vertical displacement $\delta$. The plot of experimental results has been taken from Bocca et al. [52], where it has been very roughly presented. The plot denoted MS corresponds to the numerical multi-scale solution (BC's case I). As can be observed, the structural peak load and the slope after the peak load are well estimated by the model in comparison with the experimental results. However, the structural energy dissipation is larger than that observed in the experimental result. Three DNS solutions provide an additional estimation of the MS result accuracy. The plots of the DNS solutions are rather dispersed, meaning that the numerical structural response is very sensitive with respect to the meso-scale design (aggregate distribution) close to the notches. This numerical behavior could be due to the notch geometry adopted in the present model.

Figure $18 \mathrm{~b}$ depicts a sequence of pictures (A-K) corresponding to the evolution of the cohesive cracks at the are depicted in dark red, bands which have been opening during part of the loading process but finally close are depicted in light red). (Color figure online)

macro-scale, obtained using the MS model. Finite elements in red belongs to $\Omega_{D D M}^{h}$ (state 2), and elements in green belongs to the domain $\Omega_{C S M}^{h}$ (state 1$)$. Every picture A to $\mathrm{K}$ is related to the pseudo-time instant shown in the (MS) structural response plot of Fig. 18a. Initially, two cracks start to propagate at both notch roots (picture E). However, the crack initiated in the superior notch stops, while the other continues propagating (the same phenomenology already observed in DNS solutions of Fig. 17).

The experimental report ([52]) shows one of the broken specimen, with the ratio: $c / b=0.4$. From there, it can be observed a curved crack developing from the notch root to the load application point. In the present simulation with $c / b=0.8$, the crack inducing the structural degradation is almost straight.

Figure 18c shows a sequence of pictures displaying the failure evolution, in terms of displacement fluctuations, of the mesoscopic cell associated with the macro-scale point $\mathcal{R}$. The macro-scale position of point $\mathcal{R}$ is marked in the inset of Fig. 18a. Every picture in Fig. 18c is associated with the points marked B-L in Fig. 18a, and displays the cell fracture process during the loading history. Elements in dark red correspond to bands which are in loading (open- 


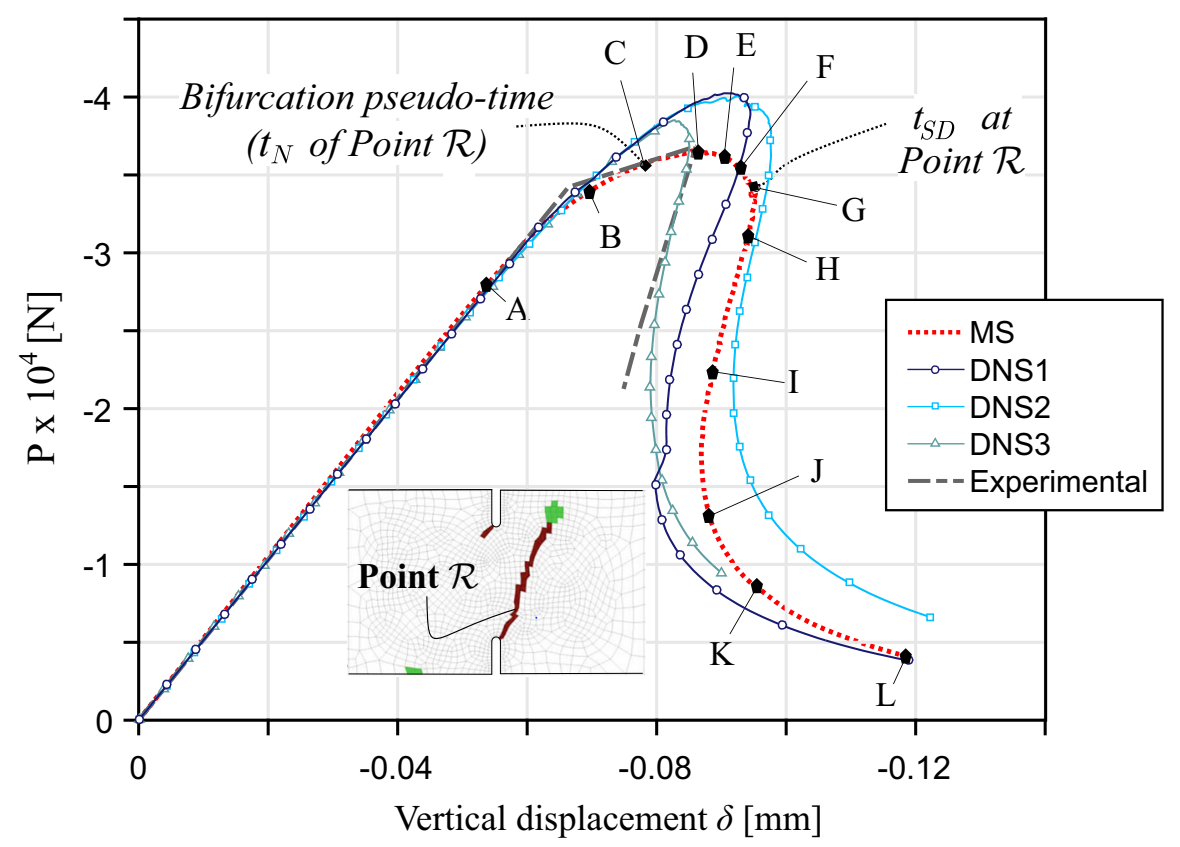

(a)

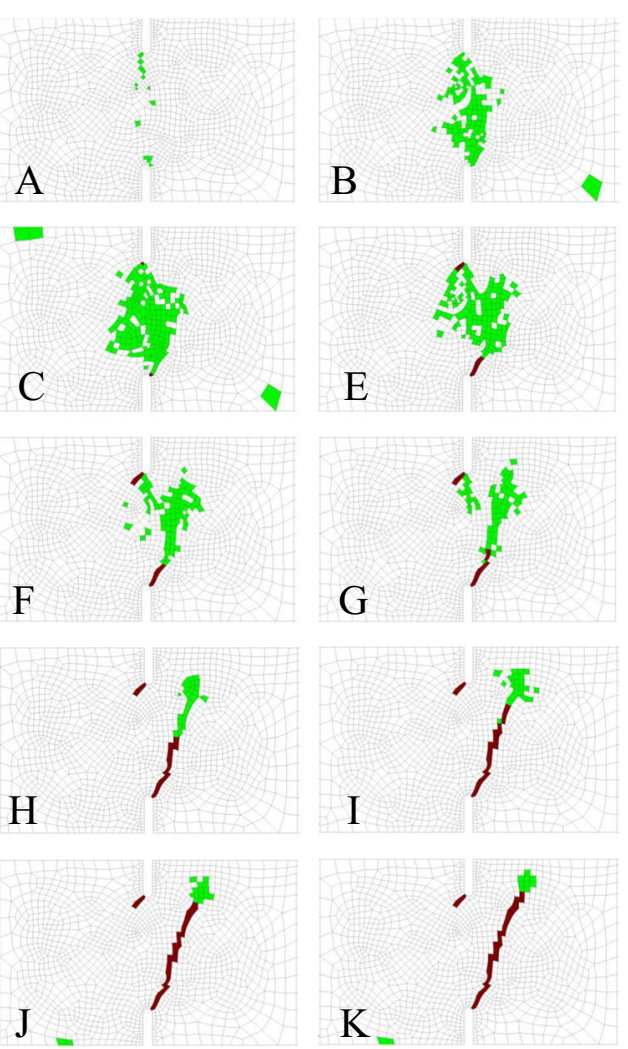

(b)

Fig. 18 Four point shear concrete specimen. a Experimental, MS and DNS structural loads $P$ versus vertical displacements $\delta$; $\mathbf{b}$ sequence of pictures showing the evolution of two cracks at the macro-structural scale (finite elements in red belongs to $\Omega_{D D M}^{h}$ (state 2), and elements in green belong to the domain $\Omega_{C S M}^{h}$ (state 1)); c sequence of pic-

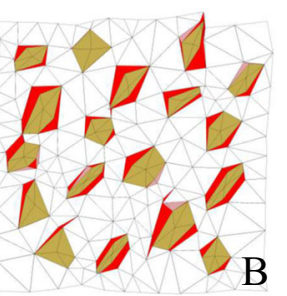

Deform. Scale $=9600$

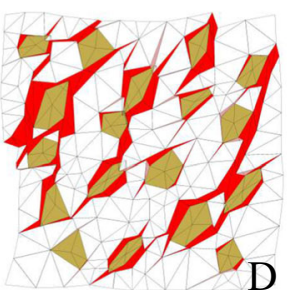

Deform. Scale $=2400$

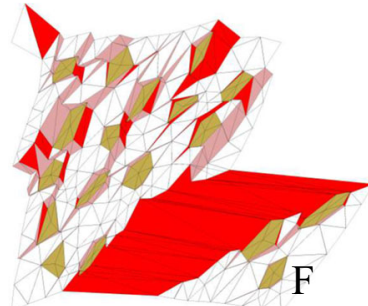

Deform. Scale $=1200$

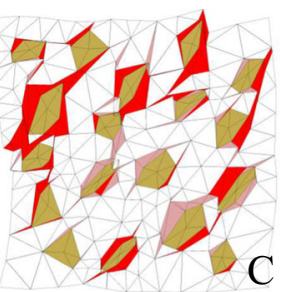

Deform. Scale $=4800$

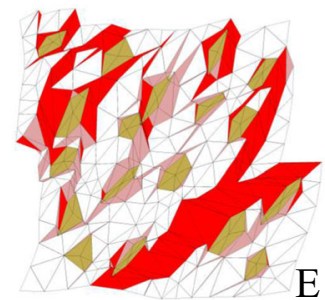

Deform. Scale $=2400$

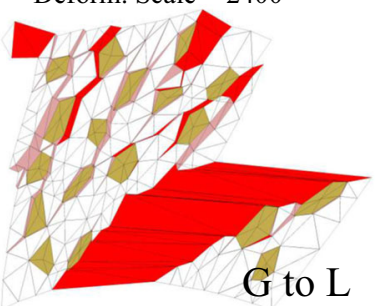

Deform. Scale $=600$

(c)

tures displaying the deformed configuration of the cells, in terms of displacement fluctuations, of the point $\mathcal{R}$ shown in the inset of Fig. 18a (Elements in dark red correspond to bands which are in loading states, elements in light red correspond to bands which are unloading). (Color figure online) 


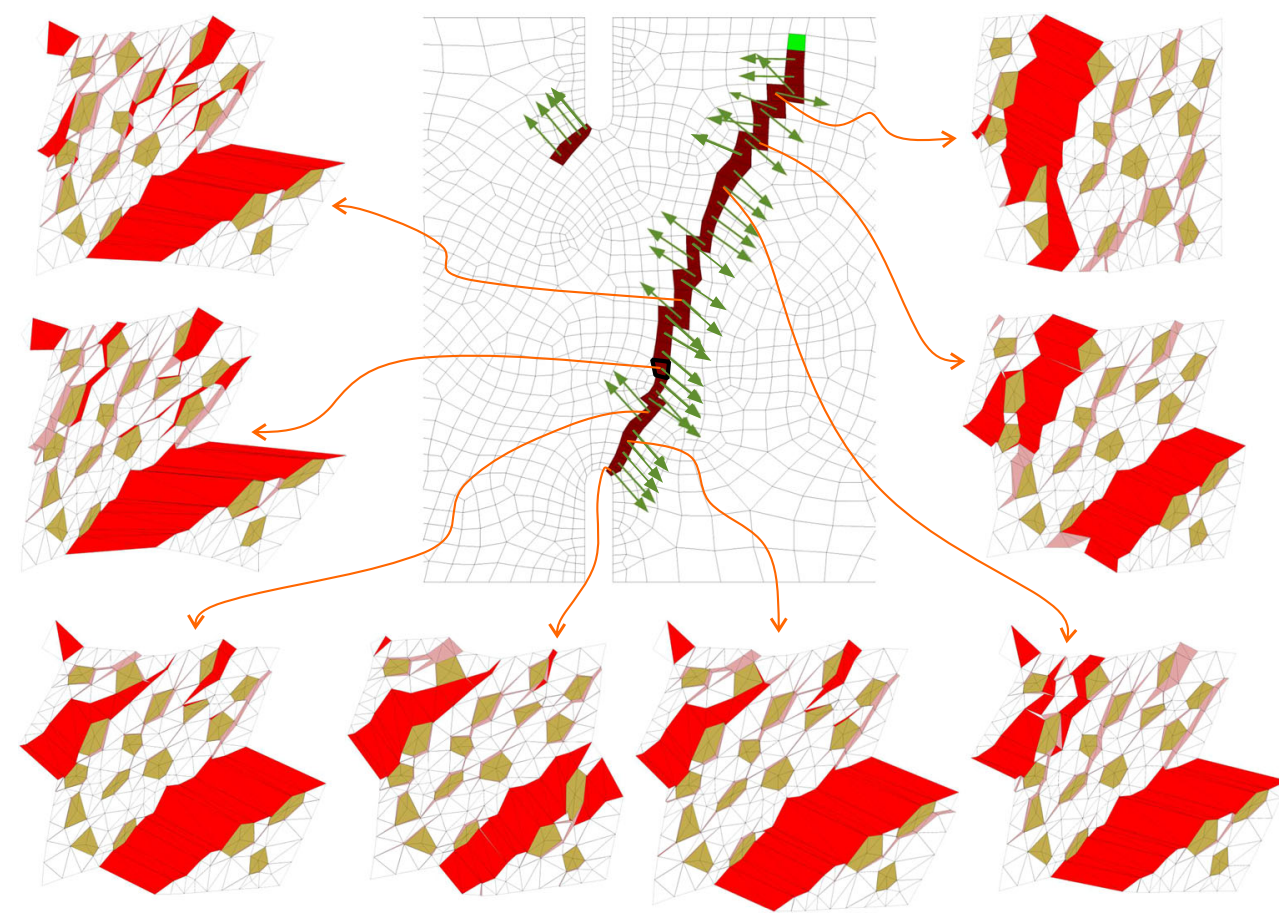

Fig. 19 Four point shear concrete specimen. Crack geometry at the macro-scale and failure mechanisms in several mesoscopic cells associated with different points along the crack. Deformed configurations of

ing) states, and elements in light red correspond to bands which are unloading (or closing). Observe the picture $\mathrm{C}$ corresponding to the instant $t_{N}$ when the finite element at the macro-scale (in $\mathcal{R}$ ) switches from state 0 to 1 . Also, it is shown the picture $\mathrm{G}$, at the instant when the strong discontinuity (SD) is embedded into the same macro-scale element (switch from state 1 to 2 ). As it is expected, the sequence of pictures shows that material degradation at point $\mathcal{R}$ starts in the ITZ elements (picture B). A complex pattern of cracks is developed (inset D-F), and at the moment of embedding the strong discontinuity at the macro-scale (plot G), the crack pattern at the mesoscopic cell is well defined. After that ( $G$ to $L$ ), the crack pattern remains fixed until the end of analysis.

Figure 19 shows the macro-scale crack path obtained with the MS model at the end of the analysis and the normal vectors $\boldsymbol{n}^{e}$ along the crack. Several deformed configurations of the mesoscopic cells, depicted in terms of the displacement fluctuation field, corresponding to different points along the macro-crack, are also shown.

\section{(b) Stress distribution}

The color maps in Fig. 20a compares the smoothed fields of principal stresses ( $\sigma_{\text {I }}$ and $\sigma_{\text {III }}$ ) obtained with the MS model and one of the DNS models. The load level $P$ at which these stress fields have been evaluated are depicted in the plot $P$ vs. $\delta$ in Fig. 20b. At that instant, the main cracks in both models the cells are depicted using displacement fluctuations and a scale factor of 600 . Distribution of normal vectors $\boldsymbol{n}^{e}$, along the crack path at the macro-scale, are shown in green arrows. (Color figure online)

have reached the positions also depicted in the inserts of the same Figure.

In Fig. 20a, the homogenized stresses of the MS model have been taken from the quadrature points placed on the regular domain of the beam. Then, they have been smoothed and finally the principal stresses have been evaluated.

The DNS model stresses have been evaluated as a spatial averaging of the stress tensor into circular domains of radius $12.5 \mathrm{~mm}$ centered at points coinciding with the nodal positions of the MS mesh. The principal stresses of these averaged tensors are depicted in Fig. 20a. Even when the MS stress distribution is a bit more noisy, it can be observed a notably good agreement between both solutions.

(c) Comparison of results obtained with MS models using different micro-cell boundary conditions

In Fig. 21, the solutions obtained with two MS models are depicted. We have compared the solutions provided by the MS model with two different types of SBC constraining the micro-cell kinematics. The type I BC's correspond to SBC with minimal kinematical constrains and the type II BC's correspond to SBC with periodic kinematical constraints.

Figure 21a plots the structural response of both simulations, while Fig. $21 \mathrm{~b}$ display the fracture at the end of analysis. 


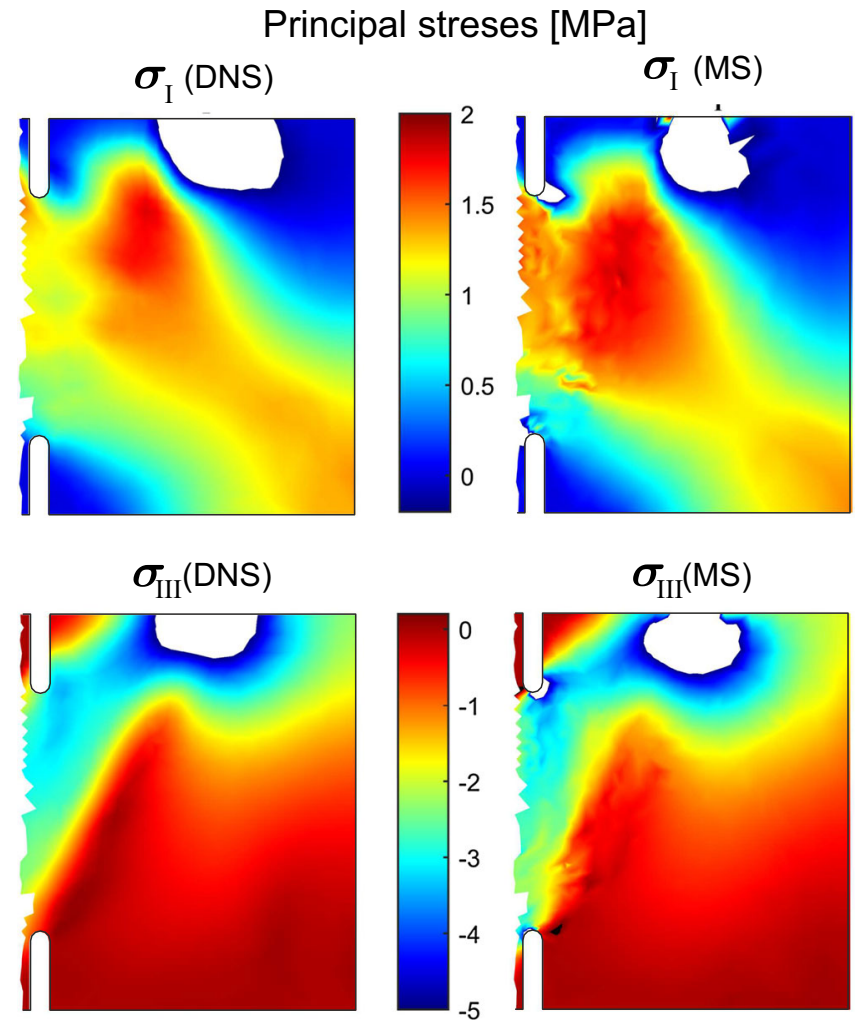

(a)

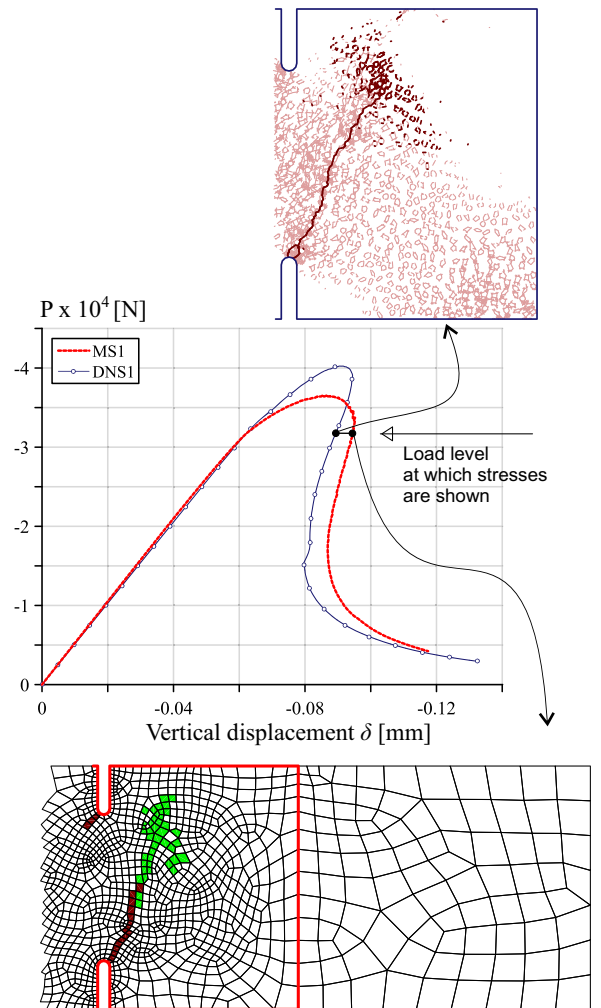

(b)
Fig. 20 Four point shear concrete specimen. Stress distribution at a given load level. a Principal stress $\left(\sigma_{\mathrm{I}}, \sigma_{\mathrm{III}}\right)$ fields obtained with DNS and MS models; $\mathbf{b}$ position of the main crack of the DNS and MS solu- tions at the instant of stress capturing in Fig. 20a. Colored domains in the MS and DNS pictures have the same meaning as in Figs. 17 and 18, respectively. (Color figure online)

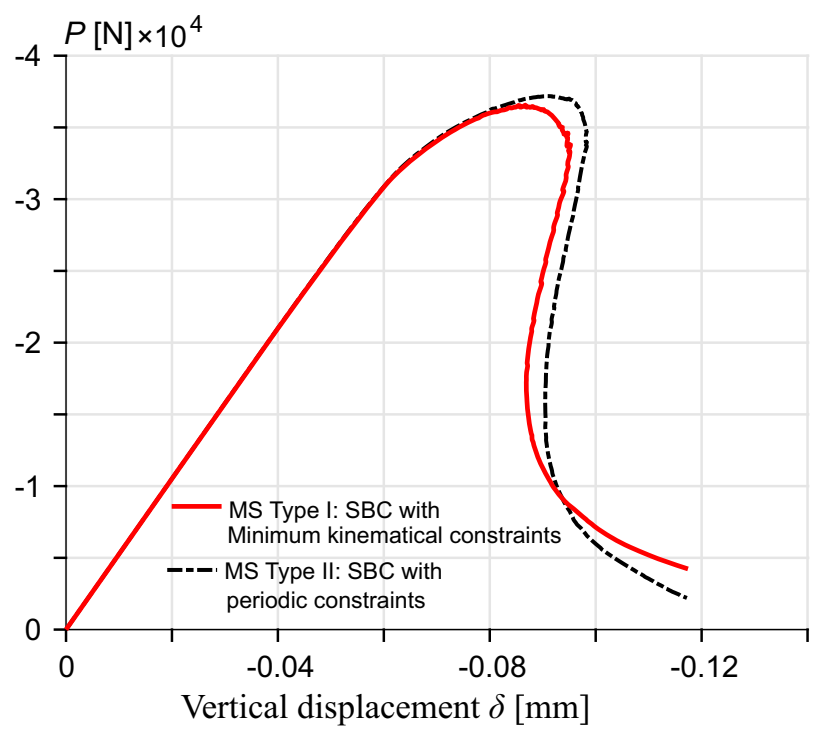

(a)
Type I: SBC with minimum constraint

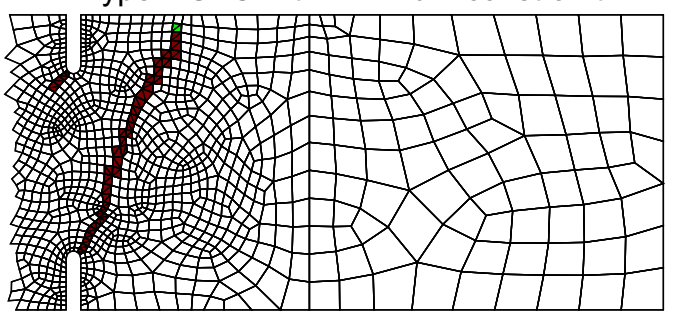

Type II: SBC with periodic constraints

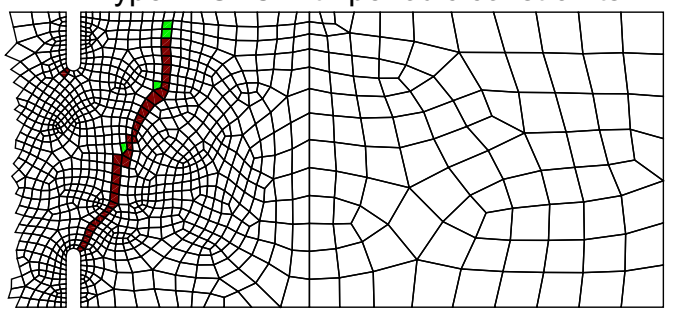

(b)
Fig. 21 Four point shear concrete specimen. Comparison of results obtained with the MS model using micro-cells with two types of SBC: type I (SBC with minimum kinematical constraints) and type II (SBC with periodic kinematical constraints). a structural responses; $\mathbf{b}$ cracks at the end of analysis (colored domains have the same meaning as in Fig. 18). (Color figure online) 


\section{Conclusion}

The main contribution of the paper focuses on describing the numerical implementation of a two-scale semi-concurrent model for fracture analysis. Cohesive surfaces are assumed at both scales of analysis.

The distinctive characteristic of this model is that it assumes two different homogenization procedures to describe the overall material response during the material degradation process:

(i) Initially, during the stable response of a point in the macro-scale, a homogenization procedure, denoted MMRp, is adopted. For a macro-scale point which is outside the influence zone of a cohesive crack, the MMRp is identical to conventional homogenization techniques previously reported in the literature. In the context of finite element implementations, the technique has been called $\mathrm{FE}^{2}$ (Feyel and Chaboche [53]). Contrarily, if the material response is stable but the macro-scale point is inside the influence zone of a cohesive surface, the macro-scale strain insertion into the RVE does not follow this kind of approach due to the influence of the tortuosity parameter $\theta$. Thus, in this case, the MMRp can be considered as a novel and non-standard homogenization procedure.

The bifurcation criteria of the macro-scale response, defined in Eq. (41), is detected using the MMRp homogenization procedure. After bifurcation, a macro-scale CSM is introduced. The cohesive forces, acting between both interfaces, decrease according with the degradation process of the overall material behavior. Therefore, the traction at the initial instant of the crack insertion is the peak stress of the resulting cohesive interface model introduced at the macro-scale.

Due to the semi-concurrent characteristic of the model, the information is transferred between scales in both directions. As can be noted, the homogenized overall stress is determined with a micro-scale analysis performed in one RVE, whose driving force is the inserted macro-scale strain.

From this observation, we conclude that the peak stress of the resulting cohesive model, which is the overall stress at the bifurcation time projected onto the surface normal vector, is strongly influenced by the macro-scale strains at that instant. This conclusion is of paramount importance when modeling ductile failure.

(ii) After detecting the singularity of the acoustic tensor at the macro-scale, a CSM is introduced. The cohesive forces are determined with a novel homogenization procedure denoted MMSp. In this case, the displacement jump across the interfaces, $\boldsymbol{\beta}$, as well as the strains at the neighbor points, $\varepsilon_{R}$, are properly inserted into the micro-scale in order to perform the analysis and finally to homogenize the overall traction vector. Again, the evolution of the cohesive force clearly depends on $\varepsilon_{R}$, and therefore, an identical conclusion to that presented in item (i) is obtained for evaluating the post-critical stage of the material degradation process.

In previous works of the authors, it has been proven that the resulting cohesive constitutive relation, traction vs. displacement jump, is objective with respect to the micro-cell size, with the condition that the micro-cell satisfies the criteria of being a RVE during the stable process of the analysis. This model property has been preserved in the new technique proposed in this paper.

A final but not less important outcome of this work is that according with the sensitivity analysis performed in this study, we conclude that:

(1) the definition of the proposed standard boundary conditions (SBC) has not affected the capturing of complex failure patterns intersecting the micro-cell at different angles, such as shown in the strips stretched at different angles (case a of Sect. 4.2);

(2) results, in terms of the homogenized traction-separation law at the macro-scale, have not been sensitive to the finite element size (case b of Sect. 4.2).

Even when the numerical assessment of the model has been performed by simulating a quasi-brittle fracture problem, it is considered that this methodology can be adapted, with minor changes, for modeling ductile failure problems in the context of a large deformation setting. This is matter of current research.

Acknowledgments S.Toro, P. J. Sánchez and A. E. Huespe acknowledge the financial support from CONICET (grant PIP 2013-2015 631) and from the European Research Council under the European Unions Seventh Framework Programme (FP/2007-2013) / ERC Grant Agreement N. 320815 (ERC Advanced Grant Project Advanced tools for computational design of engineering materials COMP-DES-MAT). P. J. Blanco and R. A. Feijóo acknowledge the financial support provided by the Brazilian agencies CNPq and FAPERJ.

\section{References}

1. Dugdale D (1960) Yielding of steel sheets containing slits. J Mech Phys Solids 8:100-108

2. Barenblatt $G$ (1962) The mathematical theory of equilibrium of cracks in brittle fracture. Adv Appl Mech 7:55-129

3. Needleman A (1987) A continuum model for void nucleation by inclusion debonding. J Appl Mech 54(3):525-531

4. Xu XP, Needleman A (1994) Numerical simulations of fast crack growth in brittle solids. J Mech Phys Solids 42:1397-1434

5. Needleman A (2014) Some issues in cohesive surface modeling. Procedia IUTAM 10:221-246 
6. Falk, ML, Needlemann A, Rice JR (2001) A critical evaluation of dynamic fracture simulation using cohesive surfaces. J Phys IV Pr-5-43-Pr-5-50

7. Xu XP, Needleman A (1995) Numerical simulations of dynamic crack growth along an interface. Int J Fract 74(4):289-324

8. Pandolfi A, Krysl P, Ortiz M (1999) Finite element simulation of ring expansion and fragmentation: the capturing of length and time scales through cohesive models of fracture. Int J Fract 95(14):279-297

9. Hutchinson JW, Evans AG (2000) Mechanics of materials: topdown approaches to fracture. Acta Mater 48(1):125-135

10. Tvergaard V (2001) Crack growth predictions by cohesive zone model for ductile fracture. J Mech Phys Solids 49(9):2191-2207

11. Siegmund T, Brocks W (2000) A numerical study on the correlation between the work of separation and the dissipation rate in ductile fracture. Eng Fract Mech 67(2):139-154

12. Huespe AE, Needleman A, Oliver J, Sánchez PJ (2009) A finite thickness band method for ductile fracture analysis. Int J Plast 25(12):2349-2365

13. Huespe AE, Needleman A, Oliver J, Sánchez PJ (2012) A finite strain, finite band method for modeling ductile fracture. Int J Plast 28(1):53-69

14. Hillerborg A, Modéer M, Petersson PE (1976) Analysis of crack formation and crack growth in concrete by means of fracture mechanics and finite elements. Cement Concr Res 6(6):773-781

15. Bažant ZP (2002) Concrete fracture models: testing and practice. Eng Fract Mech 69(2):165-205

16. Elices M, Guinea GV, Gomez J, Planas J (2002) The cohesive zone model: advantages, limitations and challenges. Eng Fract Mech 69(2):137-163

17. Oliver J, Huespe AE, Pulido MDG, Chaves E (2002) From continuum mechanics to fracture mechanics: the strong discontinuity approach. Eng Fract Mech 69:113-136

18. Oliver J (2000) On the discrete constitutive models induced by strong discontinuity kinematics and continuum constitutive equations. Int J Solids Struct 37:7207-7229

19. Tvergaard V, Hutchinson JW (1992) The relation between crack growth resistance and fracture process parameters in elasto-plastic solids. J Mech Phys Solids 40:1377-1397

20. Xia L, Shih CF (1995) Ductile crack growth I. A numerical study using computational cells with micrstructurally based length scales. J Mech Phys Solids 43:233-259

21. Vernerey FranckJ, Liu Wing Kam, Moran Brian, Olson Gregory (2008) A micromorphic model for the multiple scale failure of heterogeneous materials. J Mech Phys Solids 56(4):1320-1347

22. Sánchez PJ, Blanco PJ, Huespe AE, Feijóo RA (2013) Failureoriented multi-scale variational formulation: micro-structures with nucleation and evolution of softening bands. Comput Methods Appl Mech Eng 257:221-247

23. Gitman IM, Askes H, Sluys LJ (2007) Representative volume: Existence and size determination. Eng Fract Mech 74:2518-2534

24. Nguyen VP, Lloberas-Valls O, Stroeven M, Sluys LJ (2010a) On the existence of representative volumes for softening quasi-brittle materials - a failure zone averaging scheme. Comput Methods Appl Mech Eng 199:3028-3038

25. Belytschko T, Loehnert S, Song JH (2008) Multiscale aggregating discontinuities: a method for circumventing loss of material stability. Int J Numer Methods Eng 73(6):869-894

26. Belytschko T, Song JH (2010) Coarse-graining of multiscale crack propagation. Int J Numer Methods Eng 81(5):537-563

27. Geers MGD, Kouznetsova VG, Brekelmans WAM (2010) Multiscale computational homogenization: Trends and challenges. J Comput Appl Math 234:2175-2182

28. Bosco E, Kouznetsova VG, Geers MGD (2015) Multi-scale computational homogenization-localization for propagating discontinuities using x-fem. Int J Numer Methods Eng 102(3-4):496-527
29. Nguyen VP, Lloberas-Valls O, Sluys LJ, Stroeven M (2010b) Homogenization-based multiscale crack modelling. Comput Methods Appl Mech Eng 200:1220-1236

30. Verhoosel CV, Remmers JJC, Gutiérrez MA, de Borst R (2010) Computational homogenization for adhesive and cohesive failure in quasi-brittle solids. Int J Numer Methods Eng 83:1155-1179

31. Oliver J, Caicedo M, Roubin E, Huespe AE, Hernández JA (2015) Continuum approach to computational multiscale modeling of propagating fracture. Comput Methods Appl Mech Eng 294:384427

32. Kulkarni MG, Geubelle PH, Matous K (2009) Multi-scale modeling of heterogeneous adhesives: Effect of particle decohesion. Mech Mater 41:573-583

33. Blanco PJ, Sánchez PJ, de Souza Neto EA, Feijóo RA (2016a) Variational foundations and generalized unified theory of RVEbased multiscale models. Arch Comput Methods Eng 23:191-253. doi:10.1007/s11831-014-9137-5

34. Hill R (1972) On constitutive macro-variables for heterogeneous solids at finite strain. Proc R Soc Lond 326:131-147

35. Mandel J (1971) Plasticit classique at viscoplasticit., CISIM Lecture NotesSpringer, Berlin

36. de Souza EA (2006) Neto and R.A. Feijóo. Variational foundation on multi-scale constitutive models of solids: small and large strain kinematical formulation. LNCC Research \& Development. Report 16

37. de Souza Neto EA, Feijóo RA (2008) On the equivalence between spatial and material volume averaging of stress in large strain multiscale solid constitutive models. Mech Mater 40:803-811

38. Perić D, de Souza Neto RA, Feijóo M Partovi, Carneiro Molina AJ (2011) On micro-to-macro transitions for multi-scale analysis of non-linear heterogeneous materials. Int J Numer Methods Eng $87: 149-170$

39. de Souza Neto EA, Feijóo RA (2011) Variational foundations of large strain multiscale solid constitutive models: Kinematical formualtion. In: Vaz M, de Souza Neto EA, Muoz-Rojas PA (eds) Advanced computational materials modeling. From classical to multi-scale techniques. Wiley, Weinheim, pp 341-378

40. Blanco PJ, Sánchez PJ, de Souza Neto EA, Feijóo RA (2016b) The method of multiscale virtual power for the derivation of a secondorder mechanical model. Mech Mater 99:53-67

41. Blanco PJ, Giusti SM (2014) Thermomechanical multiscale constitutive modeling: accounting for microstructural thermal effects. J Elast 115:27-46

42. de Souza Neto EA, Blanco PJ, Sánchez PJ, Feijóo RA (2015) An rve-based multiscale theory of solids with micro-scale inertia and body force effects. Mech Mater 80:136-144

43. Toro S, Sánchez PJ, Huespe AE, Giusti SM, Blanco PJ, Feijóo RA (2014) A two-scale failure model for heterogeneous materials: numerical implementation based on the finite element method. Int J Numer Methods Eng 97(5):313-351

44. Toro S, Sánchez PJ, Blanco PJ, de Souza Neto EA, Huespe AE, Feijóo RA (2016) Multiscale formulation for material failure accounting for cohesive cracks at the macro and micro scales. Int J Plast 76:75-110

45. Simo J, Oliver J, Armero F (1993) An analysis of strong discontinuities induced by strain-softening in rate-independent inelastic solids. Comput Mech 12:277-296

46. Oliver J, Dias IF, Huespe AE (2014) Crack-path field and strain-injection techniques in computational modeling of propagating material failure. Comput Methods Appl Mech Eng 274: 289-348

47. Miehe C, Koch A (2002) Computational micro-to-macro transition of discretized microstructures undergoing small strain. Arch Appl Mech 72:300-317

48. Oliver J, Huespe AE, Blanco S, Linero DL (2005) Stability and robustness issues in numerical modeling of material failure with 
the strong discontinuity approach. Comput Methods Appl Mech Eng 195(52):7093-7114

49. Manzoli OL, Gamino AL, Rodrigues EA, Claro GKS (2012) Modeling of interfaces in two-dimensional problems using solid finite elements with high aspect ratio. Comput Struct 94:70-82

50. Carol I, López CM, Roa O (2001) Micromechanical analysis of quasi-brittle materials using fracture-based interface elements. Int J Numer Methods Eng 52(1-2):193-215
51. Unger JF, Eckardt S (2011) Multiscale modeling of concrete. Arch Comput Methods Eng 18(3):341-393

52. Bocca P, Carpinteri A, Valente S (1990) Size effects in the mixed mode crack propagation: softening and snap-back analysis. Eng Fract Mech 35(1):159-170

53. Feyel F, Chaboche JL (2000) $\mathrm{FE}^{2}$ multiscale approach for modelling the elastoviscoplastic behaviour of long fibre $\mathrm{SiC} / \mathrm{Ti}$ composite materials. Comput Methods Appl Mech Eng 183:309-330 